\title{
STRATIFICATIONS AND MACKEY FUNCTORS II: GLOBALLY DEFINED MACKEY FUNCTORS
}

\author{
PETER WEBB
}

\begin{abstract}
We describe structural properties of globally defined Mackey functors related to the stratification theory of algebras. We show that over a field of characteristic zero they form a highest weight category and we also determine precisely when this category is semisimple. This approach is used to show that the Cartan matrix is often symmetric and non-singular, and we are able to compute finite parts of it in some instances. We also develop a theory of vertices of globally defined Mackey functors in the spirit of group representation theory, as well as giving information about extensions between simple functors.
\end{abstract}

\section{INTRODUCTION}

Globally defined Mackey functors have been shown during the last 20 years or so to have applications in several directions. In [32] they were used to give a method for computing group cohomology, and also a new proof of the theorem of Benson-Feshbach and Martino-Priddy on stable decomposition of classifying spaces of finite groups. In $[12,9]$ they were used in a fundamental way in the determination of the Dade group of endopermutation modules. In $[10,11]$ applications were made in which the group of units in the Burnside ring is determined, and in which a description is given of the $G$-sets which give isomorphic rational representations.

In this paper we describe structural properties of globally defined Mackey functors related to the stratification theory of algebras. We show that over a field of characteristic zero they form a highest weight category and we also determine precisely when this category is semisimple. This approach is used to show that the Cartan matrix is often symmetric and non-singular, and we are able to compute finite parts of it in some instances. We also develop a theory of vertices of globally

2000 Mathematics Subject Classification. Primary 19A22; Secondary 16E20, $20 \mathrm{C} 20$.

Key words and phrases. Mackey functor, biset, stratification, Burnside ring, highest weight category.

Partially supported by the NSF and by MSRI. 
defined Mackey functors in the spirit of group representation theory, as well as giving information about extensions between simple functors.

The globally defined Mackey functors which we will consider depend on a choice of classes of finite groups $\mathcal{X}, \mathcal{Y}$ and $\mathcal{D}$. Fixing a commutative ring $R$ with a 1 , such a globally defined Mackey functor specifies for each group in $\mathcal{D}$ an $R$-module, and possesses operations of restriction and induction for each injective group homomorphism, as well as an operation of inflation for each surjective group homomorphism with kernel in $\mathcal{X}$, and an operation of deflation for each surjective group homomorphism with kernel in $\mathcal{Y}$. We are mainly interested in this paper in globally defined Mackey functors which are finitely generated $R$-modules at each group in $\mathcal{D}$, and every ring $R$ which we consider explicitly will be Noetherian. With these assumptions, these globally defined Mackey functors form an abelian category which we denote $\operatorname{Mack}_{R}^{\mathcal{X}, \mathcal{Y}}(\mathcal{D})$, or simply $\operatorname{Mack}_{R}^{\mathcal{X}, \mathcal{Y}}$ if we wish to suppress $\mathcal{D}$ from the notation. It will, however, be apparent that at several points the hypotheses of finite generation are unnecessary, and on occasion we point this out. The definitions will be reviewed fully in Section 2 .

In studying stratifications of $\operatorname{Mack}_{R}^{\mathcal{X}, \mathcal{Y}}(\mathcal{D})$ - in the sense of [17], for example — we require the collection of simple objects to have either a poset structure or more generally the structure of a preordered set. The simple globally defined Mackey functors were parametrized in [7] as functors $S_{H, V}$ where $H$ is a finite group in $\mathcal{D}$ taken up to isomorphism and $V$ is a simple $R \operatorname{Out}(H)$-module. There is a natural preorder on the set of such pairs $(H, V)$ where we put $(H, V) \leq(K, W)$ if and only if $K$ is isomorphic to a section of $H$, where by a section we mean a quotient of a subgroup. Taking $K=H$, this has the property that $(H, V) \leq(H, W)$ no matter what $V$ and $W$ are. We may also consider the partial order on the set of such pairs defined as the partial order for which $(H, V)<(K, W)$ if and only if $K$ is isomorphic to a proper section of $H$, this being a more stringent relation than the preorder. Neither the preorder nor the partial order we have just defined make reference to the second element $V$ in a pair $(H, V)$.

We now state one of our principal results.

Theorem 7.2. Let $R$ be a field of characteristic zero. With respect to the partial order on the simple objects defined above, and when $\mathcal{D}$ is finite, $\operatorname{Mack}_{R}^{\mathcal{X}, \mathcal{Y}}(\mathcal{D})$ is a highest weight category. When $\mathcal{D}$ is not finite, $\operatorname{Mack}_{R}^{\mathcal{X}, \mathcal{Y}}(\mathcal{D})$ is still a highest weight category, except that indecomposable injectives might conceivably not be unions of their subobjects of finite length. 
In this theorem we take the definition of highest weight category which appears in [16], and the finiteness condition on injectives is one which appears in that definition. When we are dealing with modules for a finite-dimensional algebra (as is the case in our situation when $\mathcal{D}$ is finite) this condition is automatically satisfied. In fact, $\operatorname{Mack}_{R}^{\mathcal{X}, \mathcal{Y}}(\mathcal{D})$ may be identified as the category of modules for an algebra defined in section 2 called the global Mackey algebra, and it follows when $\mathcal{D}$ is finite that this algebra is quasi-hereditary. When $\mathcal{D}$ is not finite the finiteness condition on injectives needs to be verified, and at the time of writing we have failed to do this.

This issue with finiteness conditions does not affect our calculations or applications at all, since all these may be done using suitable finite sets of groups $\mathcal{D}$ and - as we show in section 7 - the highest weight structure on $\operatorname{Mack}_{R}^{\mathcal{X}, \mathcal{Y}}(\mathcal{D})$ is compatible with restricting the domain of definition from $\mathcal{D}$ to smaller collections of groups. Thus we can deduce the validity of BGG reciprocity, for example, which is used in a key way in section 8 .

The proof of Theorem 7.2 is the culmination of several sections where we study the globally defined Mackey functors $\Delta_{H, U}$ which play the role of the standard objects in the highest weight category, and also the dually defined functors $\nabla_{H, U}$. These functors are defined in Section 5 , first in an explicit manner and then by showing that they have a certain adjoint property. We establish many of their properties, including in Theorem 6.3 the fact that over an arbitrary ring $R$ (not just a field of characteristic zero), each projective globally defined Mackey functor has a filtration with factors $\Delta_{H, U}$ where $U$ is a direct summand of a permutation module for $R \operatorname{Out}(H)$. Later in Proposition 6.6 strong forms of the usual Ext properties between the functors $\Delta$ and the dually constructed functors $\nabla$ are given, while earlier in Theorem 5.10 it is shown that Ext groups between simple functors may be computed within the $\Delta$ and $\nabla$ functors.

In the case of ordinary Mackey functors (which have no inflation or deflation operations) over a field of characteristic zero the functors $\Delta_{H, V}$ are simple and in fact the category of Mackey functors is semisimple (see [30]). For globally defined Mackey functors this need not be the case, as indicated in the following result.

Theorem 9.5. Let $R$ be a field of characteristic zero and all the class of all finite groups. Then $\operatorname{Mack}_{R}^{\mathcal{X}, \mathcal{Y}}$ (all) is semisimple if and only if $\mathcal{X}=\mathcal{Y}=1$. 
This result implies the semisimplicity of endomorphism rings of globally defined Mackey functors when $\mathcal{X}=\mathcal{Y}=1$, and these rings include the Grothendieck rings of bisets which have trivial stabilizers on each side. This implication is presented in Theorem 9.6.

We are able to use the fact that $\operatorname{Mack}_{R}^{\mathcal{X}, \mathcal{Y}}(\mathcal{D})$ is a highest weight category when $R$ is a field of characteristic zero and $\mathcal{D}$ is finite to compute the Cartan matrix of globally defined Mackey functors over an arbitrary field. This is done in Section 8 and some specific Cartan matrices are computed in Section 11. The calculation exploits two techniques, the first being BGG reciprocity, which applies whenever we have a highest weight category. We combine this with the 'BrauerCartan square' of Geck and Rouquier [22]. This is a generalized version of the decomposition theory of Brauer, extended to a situation where in characteristic zero we have an algebra which is not semisimple. Using these methods we are able to prove:

Theorem 8.5. Let $R$ be an algebraically closed field and suppose that $\mathcal{X}=\mathcal{Y}$. Then the Cartan matrix of globally defined Mackey functors is symmetric.

Theorem 8.6. Let $R$ be a field and in case $R$ has positive characteristic suppose that $\mathcal{X}=\mathcal{Y}$. Then the Cartan matrix of globally defined Mackey functors is non-singular.

The Cartan matrix is infinite, and by 'non-singular' we mean that the columns of the matrix are linearly independent. We speculate that the Cartan matrix is non-singular in all cases without the restriction that $\mathcal{X}=\mathcal{Y}$ in positive characteristic, but our proof fails to establish this.

As a corollary of Theorem 7.2 we have an application to classifying spaces of finite groups. We denote by $\left(B G_{+}\right)_{p}^{\wedge}$ the $p$-completed spectrum obtained from the classifying space $B G$ of $G$ with a disjoint point adjoined. We are interested in stable homotopy classes of maps $\left[\left(B G_{+}\right)_{p}^{\wedge},\left(B H_{+}\right)_{p}^{\wedge}\right]$.

Corollary10.2. Let $\mathcal{D}$ be a finite set of groups closed under taking sections and let $p$ be a prime. Then the p-complete spectrum

$$
B=\bigvee_{G \in \mathcal{D}}\left(B G_{+}\right)_{p}^{\wedge}
$$

has the property that the algebra $[B, B] \otimes_{\mathbb{Z}_{p}} \mathbb{Q}_{p}$ is quasi-hereditary. Here $\mathbb{Z}_{p}$ and $\mathbb{Q}_{p}$ denote the p-adic integers and the p-adic rationals.

As explained in [32], the full subcategory of the category of spectra whose objects are wedge sums of summands of the the spectra $\left(B G_{+}\right)_{p}^{\wedge}$, 
is equivalent to a full subcategory of $\mathrm{Mack}_{\mathbb{Z}_{p}}^{\text {all,1}}$ whose objects are certain projective functors, and this is the connection between Corollary 10.2 and Theorem 7.2. We apply the theory in Section 11.4 to compute the Cartan matrices both of $[B, B] \otimes_{\mathbb{Z}_{p}} \mathbb{Q}_{p}$ and of $[B, B] \otimes_{\mathbb{Z}_{p}} \mathbb{F}_{p}$ when $p=2$ and $\mathcal{D}=\left\{1, C_{2}, C_{4}, C_{8},\left(C_{2}\right)^{2}, D_{8}, Q_{8}\right\}$.

Prior to all this in our exposition, we introduce in Section 3 a theory of relative projectivity for globally defined Mackey functors. This is defined with respect to changes in the class of groups $\mathcal{D}$ on which the functors are defined, using the notion of induction $\uparrow_{\mathcal{E}}^{\mathcal{D}}$ and restriction $\downarrow_{\mathcal{E}}^{\mathcal{D}}$ between $\operatorname{Mack}_{R}^{\mathcal{X}, \mathcal{Y}}(\mathcal{E})$ and $\operatorname{Mack}_{R}^{\mathcal{X}, \mathcal{Y}}(\mathcal{D})$ when $\mathcal{E} \subseteq \mathcal{D}$. Relative projectivity is defined in the usual way using these operations and the main result here is the following.

Theorem 3.9. Let $M$ be a globally defined Mackey functor. There is a unique minimal set of groups $\mathcal{E}$, closed under taking sections, relative to which $M$ is projective. Furthermore $M \cong M \downarrow_{\mathcal{E}}^{\mathcal{D}} \uparrow_{\mathcal{E}}^{\mathcal{D}}$, and $M \downarrow_{\mathcal{E}}^{\mathcal{D}}$ is (up to isomorphism) the only Mackey functor $N$ defined on $\mathcal{E}$ with the property that $M \cong N \uparrow_{\mathcal{E}}^{\mathcal{D}}$.

We call the minimal set $\mathcal{E}$ the vertex of $M$. This result does not depend on the ground $\operatorname{ring} R$ or require that $M$ be indecomposable, unlike the case of group representations. We also show - again, unlike the case of group representations - that induction from and restriction to a vertex are inverse operations. We determine the vertices of the projective functors in Proposition 3.10 and of the standard functors $\Delta$ in Corollary 5.4. In Proposition 5.11 we provide a condition on the vertices of simple functors for there to be a non-split extension between them.

In Section 2 we review definitions and basic lemmas, and give a proof of the equivalence of the definitions of globally defined Mackey functors by means of axioms on the one hand, and as functors on a certain category on the other hand. Later in Section 4 we discuss the ascending and descending filtration of globally defined Mackey functors which are fundamental in the highest weight category structure, and we relate them to the constructions of Brauer quotient and its dual, the restriction kernel.

We take the opportunity to comment on the different terminology which has been used for the functors which we here call globally defined Mackey functors. The same terminology in this generality was used in [33]. On page 278 of [18] these functors are introduced in the special case $\mathcal{Y}=1$, where they are called 'global Mackey functors'. These same functors with the condition $\mathcal{Y}=1$ appear in [28] where they are 
called 'functors with Mackey structure' and also in [32] where they are called 'inflation functors'. In the last reference the functors which arise when $\mathcal{X}=\mathcal{Y}=1$ are called 'global Mackey functors'. In $[9,10,11]$ the term 'biset functors' is used in a situation where $\mathcal{X}$ and $\mathcal{Y}$ are both taken to be all finite groups. The term 'globally defined Mackey functor' is intended to include all of the special cases, and they are all considered in this paper. It seems to the present author that another good possibility would be to use 'global Mackey functor' as the term which includes all the special cases, but one would have to ignore the fact that this has already been used twice to mean different things.

\section{Definitions of GLOBAlly DEFined MaCKEy FUnCtors AND PRELIMINARY LEMMAS}

Globally defined Mackey functors may be defined in several ways: as functors on a certain category, as modules for an algebra, and by means of axioms. In this section we review the notation and definitions we will use and provide a proof of the equivalence of the axiomatic definition with the functorial definition, since this has not appeared in print before. Lemmas from this section will be crucial later on.

By a section of a group $G$ we mean a homomorphic image of a subgroup of $G$. We will often refer to a 'set of groups closed under taking sections', and by this we mean a set of groups with the property that every section of every group in the set is isomorphic to a group in the set. Strictly speaking it is abuse of terminology since a set of groups which is genuinely closed under taking sections contains every group isomorphic to a group in the set, and so is no longer a set. We will refer to sets of groups which are closed under taking extensions in a similar spirit. The groups we consider in such a context will always be finite.

We define a generalization of the double Burnside ring, which has its origins in [37], [27], [25] and [1]. Let $G$ and $H$ be finite groups. By a $(G, H)$-biset we mean a set $\Omega={ }_{G} \Omega_{H}$ with commuting actions of $G$ and $H$, with $G$ acting from the left and $H$ from the right. This is the same as specifying a $G \times H$-set, since we may employ the device of converting the right $H$-action into a left action by means of the antiautomorphism $h \mapsto h^{-1}$. Let $\mathcal{X}$ and $\mathcal{Y}$ be sets of finite groups closed under taking sections and extensions. We define $A_{\mathbb{Z}}^{\mathcal{X}, \mathcal{Y}}(G, H)$ to be the Grothendieck group of finite $(G, H)$-bisets which have the property that the stabilizer in $G$ of each point in $\Omega$ lies in $\mathcal{X}$, and the stabilizer in $H$ of each point in $\Omega$ lies in $\mathcal{Y}$. Thus $A_{\mathbb{Z}}^{\mathcal{X}, \mathcal{Y}}(G, H)$ is a free abelian group with basis the transitive such bisets (transitive with respect to the action of $G \times H$ ), 
and in this group we identity a non-transitive biset with the sum of the bisets given by its orbits. Fixing a commutative ring $R$ with a 1 we put $A_{R}^{\mathcal{X}, \mathcal{Y}}(G, H)=R \otimes_{\mathbb{Z}} A_{\mathbb{Z}}^{\mathcal{X}, \mathcal{Y}}(G, H)$. The special case $A_{R}^{\text {all,1 }}(G, G)$ is the double Burnside ring, where 'all' means a set of finite groups representing every isomorphism class, and 1 is a set containing just a group with one element.

We next define a category $\mathcal{C}_{R}^{\mathcal{X}, \mathcal{Y}}(\mathcal{D})$ which is a more general form of a category defined in $[27,1]$ where it is called the Burnside category. In generality it was considered by Bouc [7]. Let $\mathcal{D}$ be a set of finite groups closed under taking sections. The objects of $\mathcal{C}_{R}^{\mathcal{X}, \mathcal{Y}}(\mathcal{D})$ are the groups in $\mathcal{D}$. If $G$ and $H$ are such groups we define the morphisms in $\mathcal{C}_{R}^{\mathcal{X}, \mathcal{Y}}(\mathcal{D})$ from $H$ to $G$ to be $\operatorname{Hom}(H, G)=A_{R}^{\mathcal{X}, \mathcal{Y}}(G, H)$, a convention which makes sense since we will be applying morphisms from the left. Thus this set of morphisms is a free $R$-module with basis the transitive bisets ${ }_{G} \Omega_{H}$ whose $G$-stabilizers lie in $\mathcal{X}$ and whose $H$-stablizers lie in $\mathcal{Y}$. Composition of morphisms is defined to be $R$-bilinear, and on homomorphisms which are actually bisets it is defined to be the amalgamated product:

$$
{ }_{G} \Omega_{H} \circ{ }_{H} \Psi_{K}:=\Omega \times{ }_{H} \Psi,
$$

where $\Omega \times_{H} \Psi$ means the set of orbits of $H$ on $\Omega \times \Psi$ under the action $h(\omega, \psi)=\left(\omega h^{-1}, h \psi\right)$. We let $\operatorname{Mack}_{R}^{\mathcal{X}, \mathcal{Y}}(\mathcal{D})$ denote the category of $R$-linear functors $\mathcal{C}_{R}^{\mathcal{X}, \mathcal{Y}}(\mathcal{D}) \rightarrow R$-mod. These are the functors we call globally defined Mackey functors. Many of the definitions and constructions we will give, such as of the functors $\Delta$, are the same regardless of what $\mathcal{D}$ is and on occasion we suppress $\mathcal{D}$ from the notation.

Globally defined Mackey functors may be regarded as modules for an algebra $\mu_{R}^{\mathcal{X}, \mathcal{Y}}(\mathcal{D})$ which we term the global Mackey algebra. The general construction of such an algebra starting from an additive category can be traced back to [21, Chap. II], and we describe it in our particular case. Working with a set of finite groups $\mathcal{D}$ which is closed under taking sections we put

$$
\mu_{R}^{\mathcal{X}, \mathcal{Y}}(\mathcal{D})=\bigoplus_{G, H \in \mathcal{D}} A_{R}^{\mathcal{X}, \mathcal{Y}}(G, H)
$$

as an $R$-module, and define the multiplication of two elements which lie in these summands to be the same as composition in $\mathcal{C}_{R}^{\mathcal{X}, \mathcal{Y}}(\mathcal{D})$ if the elements can be composed, and zero otherwise. It is immediate that a globally defined Mackey functor $M$ may be regarded as a $\mu_{R}^{\mathcal{X}, \mathcal{Y}}(\mathcal{D})$ module $\bigoplus_{H \in \mathcal{D}} M(H)$, with the action of a biset ${ }_{G} \Omega_{H}$ on the summand $M(H)$ being given by $M\left({ }_{G} \Omega_{H}\right)$, and zero on the other summands. Conversely, for each group $G$ in $\mathcal{D}$ there is an idempotent ${ }_{G} G_{G} \in$ 
$A_{R}^{\mathcal{X}, \mathcal{Y}}(G, G) \subseteq \mu_{R}^{\mathcal{X}, \mathcal{Y}}(\mathcal{D})$, and each $\mu_{R}^{\mathcal{X}, \mathcal{Y}}(\mathcal{D})$-module $U$ gives rise to a globally defined Mackey functor $M$ where $M(G)={ }_{G} G_{G} \cdot U$. This procedure produces an equivalence of categories between $\operatorname{Mack}_{R}^{\mathcal{X}, \mathcal{Y}}(\mathcal{D})$ and $\mu_{R}^{\mathcal{X}, \mathcal{Y}}(\mathcal{D})$-modules $U$ which have the property that $U=\bigoplus_{G \in \mathcal{D}} G_{G} G_{G}$. $U$. Observe that $\mu_{R}^{\mathcal{X}, \mathcal{Y}}(\mathcal{D})$ possesses an identity element if and only if $\mathcal{D}$ is finite, the identity element being $e_{\mathcal{D}}=\sum_{G \in \mathcal{D}} G_{G}$.

It is useful to define globally defined Mackey functors by means of axioms, and such a set of such axioms was given in [33], extending axioms given for more restrictive kinds of functors in [32]. We will use these axioms in Section 5 to show that the functors $\Delta_{H, V}$ we construct are indeed globally defined Mackey functors. We repeat the axioms from [33].

Let $\mathcal{X}$ and $\mathcal{Y}$ be sets of finite groups closed under taking sections and extensions, let $\mathcal{D}$ be a set of finite groups closed under taking sections, and let $R$ be a commutative ring. We say that a globally defined Mackey functor over $R$, defined on $\mathcal{D}$, with respect to $\mathcal{X}$ and $\mathcal{Y}$, is a specification $M$ which consists of an $R$-module $M(G)$ for each finite group $G$, together with for each homomorphism $\alpha: G \rightarrow K$ with Ker $\alpha \in \mathcal{Y}$ an $R$-module homomorphism $\alpha_{*}: M(G) \rightarrow M(K)$ and for each homomorphism $\beta: G \rightarrow K$ with $\operatorname{Ker} \beta \in \mathcal{X}$ an $R$-module homomorphism $\beta^{*}: M(K) \rightarrow M(G)$. These morphisms should satisfy the following relations:

(1) $(\alpha \gamma)_{*}=\alpha_{*} \gamma_{*}$ and $(\beta \delta)^{*}=\delta^{*} \beta^{*}$ always, whenever these are defined;

(2) whenever $\alpha: G \rightarrow G$ is an inner automorphism then $\alpha_{*}=1=$ $\alpha^{*}$

(3) for every commutative diagram of groups

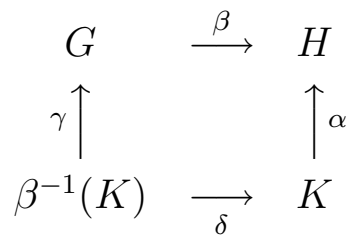

in which $\alpha$ and $\gamma$ are inclusions and $\beta$ and $\delta$ are surjections we have $\alpha^{*} \beta_{*}=\delta_{*} \gamma^{*}$ whenever $\operatorname{Ker} \beta \in \mathcal{Y}$, and $\beta^{*} \alpha_{*}=\gamma_{*} \delta^{*}$ whenever $\operatorname{Ker} \beta \in \mathcal{X}$;

(4) for every commutative diagram

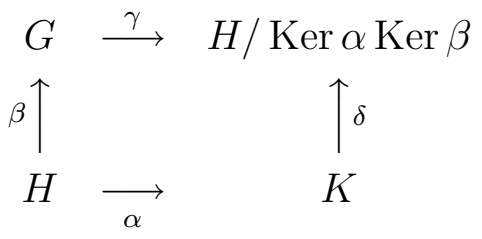


in which $\alpha, \beta, \gamma$ and $\delta$ are all surjections, with $\operatorname{Ker} \beta \in \mathcal{Y}$ and Ker $\alpha \in \mathcal{X}$, we have $\beta_{*} \alpha^{*}=\gamma^{*} \delta_{*}$;

(5) (Mackey axiom) for every pair of subgroups $G, K \leq H$ of every group $H$ we have

$$
\left(\iota_{K}^{H}\right)^{*}\left(\iota_{G}^{H}\right)_{*}=\sum_{h \in[K \backslash H / G]}\left(\iota_{K \cap h}^{K}\right)_{*} c_{h *}\left(\iota_{K}^{G} \cap G\right) *
$$

where $\iota_{K}^{H}: K \hookrightarrow H$ and $\iota_{G}^{H}: G \hookrightarrow H$ etc. are the inclusion maps and $c_{h}: K^{h} \cap G \rightarrow K \cap{ }^{h} G$ is the homomorphism $c_{h}(x)=h x h^{-1}$.

We should note that certain things which are required for these axioms to make sense, do in fact hold. Thus in both (3) and (4), if $\operatorname{Ker} \beta \in \mathcal{Y}$ then Ker $\delta \in \mathcal{Y}$ also; in (1), if Ker $\alpha \in \mathcal{Y}$ and Ker $\gamma \in \mathcal{Y}$ then Ker $\alpha \gamma \in \mathcal{Y}$ also; and so on. The axiom here which did not appear in [32] is (4), and it implies in particular that if $\alpha: H \rightarrow K$ is an isomorphism, then $\left(\alpha^{-1}\right)_{*}=\alpha^{*}$ and $\left(\alpha^{-1}\right)^{*}=\alpha_{*}$.

We will indicate a proof that the definition of a globally defined Mackey functor just given is really the same as the definition given in terms of bisets. The key to seeing this is Bouc's lemma which decomposes bisets into bisets of a certain kind which on the next pages we will call special, and which we now define. Suppose we have a group homomorphism $G \rightarrow K$. We may regard the set $K$ as a $(G, K)$-biset ${ }_{G} K_{K}$ with $G$ acting from the left by first applying the homomorphism to $K$ and then multiplying from the left within $K$, and with $K$ acting by right multiplication. In a similar way we may also obtain from this homomorphism a $(K, G)$-biset ${ }_{K} K_{G}$. By a special biset we mean any biset of the form ${ }_{G} K_{K}$ or ${ }_{K} K_{G}$ where the homomorphism $G \rightarrow K$ is required to be either the inclusion of a subgroup or an epimorphism.

Using these special bisets we may see how a functor in $\operatorname{Mack}_{R}^{\mathcal{X}, \mathcal{Y}}(\mathcal{D})$ gives rise to a functor which satisfies the above axioms, and vice-versa. For, given $F \in \operatorname{Mack}_{R}^{\mathcal{X}, \mathcal{Y}}(\mathcal{D})$, we may define $M(G)=F(G)$ on finite groups $G$; whenever $\alpha: G \rightarrow K$ with $\operatorname{Ker} \alpha \in \mathcal{Y}$ we define $\alpha_{*}=$ $F\left({ }_{K} K_{G}\right): M(G) \rightarrow M(K)$; and whenever $\beta: G \rightarrow K$ with $\operatorname{Ker} \beta \in \mathcal{X}$ we define $\beta^{*}=F\left({ }_{G} K_{K}\right): M(K) \rightarrow M(G)$. The fact that $M$ satisfies the axioms listed above is now a consequence of the following easilyverified isomorphisms of bisets: for axiom (1), ${ }_{H} H_{G}{ }^{\circ}{ }_{G} G_{K} \cong{ }_{H} H_{K}$ and its reverse ${ }_{K} G_{G} \circ{ }_{G} H_{H} \cong{ }_{K} H_{H}$; for axiom (2), if $\alpha: G \rightarrow G$ is inner then ${ }_{G} G_{G}$ with $G$ acting via $\alpha$ on one side and straight multiplication on the other side is isomorphic to ${ }_{G} G_{G}$ with $G$ acting via straight multiplication on both sides; for axiom (3), ${ }_{K} H_{H} \circ{ }_{H} H_{G} \cong{ }_{K} K_{\beta^{-1}(K)} \circ$ $\beta^{-1}(K) G_{G}$ and its reverse when $\beta: G \rightarrow H$ is epi and $K \leq H$; for axiom (4), writing $L=H / \operatorname{Ker} \alpha \operatorname{Ker} \beta$ we have ${ }_{G} L_{L} \circ{ }_{L} L_{K} \cong{ }_{G} G_{H} \circ{ }_{H} K_{K}$. 
Finally if $G, K \leq H$ we have ${ }_{K} H_{H} \circ{ }_{H} H_{G} \cong \bigcup_{h \in[K \backslash H / G]} K_{K} K_{K^{h} \cap G} \circ$ $K^{h} \cap G G_{G}$ which gives axiom (5).

It is conversely the case that an object $M$ satisfying the above axioms $(1)$ - (5) gives rise to a functor $F$ in $\operatorname{Mack}_{R}^{\mathcal{X}, \mathcal{Y}}(\mathcal{D})$, and this part of the argument uses the following lemma of Bouc. It is closely related to a result known as 'Goursat's lemma' and which can be found as Exercise 8 to Chapter 1, Section 4 of [13].

Lemma 2.1. (Bouc [7]) Let $G$ and $K$ be finite groups. Every transitive $(G, K)$-biset $\Omega$ is isomorphic to one of the form ${ }_{G} G_{A} \circ_{A} H_{H} \circ_{H} H_{B} \circ_{B} K_{K}$ with $A \leq G, B \leq K$ and $H$ a homomorphic image of each of $A$ and $B$ : $A \rightarrow H \leftarrow B$. Furthermore $\operatorname{Stab}_{G}(\omega) \in \mathcal{X}$ for all $\omega$ in $\Omega$ if and only if $\operatorname{Ker}(A \rightarrow H) \in \mathcal{X}$, and $\operatorname{Stab}_{K}(\omega) \in \mathcal{Y}$ for all $\omega$ in $\Omega$ if and only if $\operatorname{Ker}(B \rightarrow H) \in \mathcal{Y}$.

Using this result, if we are given an object $M$ satisfying axioms (1) - (5) above we may define a functor $F \in \operatorname{Mack}_{R}^{\mathcal{X}, \mathcal{Y}}(\mathcal{D})$ as follows: on objects we put $F(G)=M(G)$. We may write any morphism $\Omega: K \rightarrow$ $G$ in the form $\Omega=\sum_{i=1}^{t} \lambda_{i} \Omega_{i}$ where $\lambda_{i} \in R$ and the $\Omega_{i}$ are transitive $(G, K)$-bisets. We put $F(\Omega)=\sum_{i=1}^{t} \lambda_{i} F\left(\Omega_{i}\right)$ where the $F\left(\Omega_{i}\right)$ are defined as follows. We write $\Omega_{i} \cong{ }_{G} G_{A_{i}} \circ{ }_{A_{i}} H_{i H_{i}} \circ{ }_{H_{i}} H_{i B_{i}} \circ{ }_{B_{i}} K_{K}$ using Bouc's Lemma where $G \stackrel{\alpha_{i}}{\longleftrightarrow} A_{i} \stackrel{\beta_{i}}{\rightarrow} H_{i} \stackrel{\gamma_{i}}{\longleftarrow} B_{i} \stackrel{\delta_{i}}{\longleftrightarrow} K$; we set $F\left(\Omega_{i}\right)=\alpha_{i *} \beta_{i}^{*} \gamma_{i *} \delta_{i}^{*}$.

We now need to explain why it is that the construction of $F$ just given is independent of choices, and does indeed specify a functor. The independence of choices will follow from the next lemma which is also crucial in later sections.

Lemma 2.2. Let $A$ and $U$ be subgroups of $G$, let $B$ and $W$ be subgroups of $K$, and suppose we are given morphisms $G \stackrel{\alpha}{\leftrightarrow} A \stackrel{\beta}{\leftrightarrow} H \stackrel{\gamma}{\longleftarrow} B \stackrel{\delta}{\hookrightarrow} K$ and $G \stackrel{\pi}{\longleftarrow} U \stackrel{\rho}{\rightarrow} V \stackrel{\sigma}{\longleftarrow} W \stackrel{\tau}{\hookrightarrow} K$ where $\alpha, \delta, \pi, \tau$ are inclusion morphisms and $\beta, \gamma, \rho, \sigma$ are surjections. Then

$$
{ }_{G} G_{A} \circ{ }_{A} H_{H} \circ{ }_{H} H_{B} \circ{ }_{B} K_{K} \cong{ }_{G} G_{U} \circ{ }_{U} V_{V} \circ{ }_{V} V_{W} \circ{ }_{W} K_{K}
$$

as $(G, K)$-bisets if and only if there is a commutative diagram

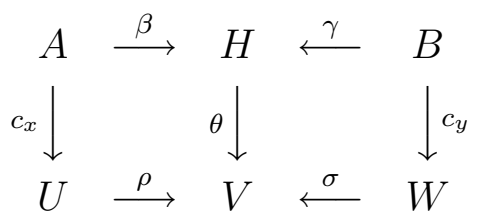

for some elements $x \in G, y \in K$ and a group isomorphism $\theta$. Here $c_{x}$ and $c_{y}$ denote conjugation homomorphisms and $U={ }^{x} A, W={ }^{y} B$. 
Proof. It is convenient to identify each $(G, K)$-biset $\Omega$ as a left $G \times K$ set where the action of $G \times K$ on $\Omega$ is given by $(g, k) \omega=g \omega k^{-1}$. Under this identification, if $\Omega$ is a transitive set then $\Omega \cong G \times K / \operatorname{Stab}_{G \times K}(\omega)$ where $\omega$ may be taken to be any element of $\Omega$. Writing the elements of the composite ${ }_{G} G_{A^{\circ}{ }_{A}} H_{H}{ }^{\circ} H_{B} H_{B}{ }_{B} K_{K}$ as equivalence classes $\left[g, h_{1}, h_{2}, k\right]$ of quadruples $\left(g, h_{1}, h_{2}, k\right)$ where $g \in G, h_{1}, h_{2} \in H$ and $k \in K$, we have

$$
\operatorname{Stab}_{G \times K}([1,1,1,1])=\left\{(g, k) \mid\left(g, 1,1, k^{-1}\right) \sim(1,1,1,1)\right\} .
$$

Now $\left(g, 1,1, k^{-1}\right) \sim(1,1,1,1)$ entails $g \in A$ and $k \in K$, and then $\left(g, 1,1, k^{-1}\right) \sim\left(1, \beta(g), \gamma\left(k^{-1}\right), 1\right) \sim\left(1, \beta(g) \gamma\left(k^{-1}\right), 1,1\right)$, which is equivalent to $(1,1,1,1)$ precisely if $\beta(g)=\gamma(k)$. Thus

$$
\operatorname{Stab}_{G \times K}([1,1,1,1])=\{(g, k) \in A \times B \mid \beta(g)=\gamma(k)\} .
$$

Using the fact that $G \times K$-sets of the form $G \times K / \operatorname{Stab}(\omega)$ are isomorphic if and only if the stabilizer groups are conjugate, we see that

$$
{ }_{G} G_{A} \circ{ }_{A} H_{H} \circ{ }_{H} H_{B} \circ{ }_{B} K_{K} \cong{ }_{G} G_{U} \circ{ }_{U} V_{V} \circ{ }_{V} V_{W} \circ{ }_{W} K_{K}
$$

if and only if $\{(g, k) \in A \times B \mid \beta(g)=\gamma(k)\}$ is conjugate to $\{(g, k) \in$ $U \times W \mid \rho(g)=\sigma(k)\}$. This happens precisely if there exist $x \in G$ and $y \in K$ with ${ }^{x} A=U$ and ${ }^{y} B=W$ so that

$$
\beta(g)=\gamma(k) \Leftrightarrow \rho\left({ }^{x} g\right)=\sigma\left({ }^{y} k\right) \quad \text { for all } g \in A \text { and } k \in B \text {. }
$$

If there exists a commutative diagram as in the statement of the lemma then this condition is clearly satisfied. Conversely, if $(*)$ is satisfied and $g \in A$ then $\beta(g)=1=\gamma(1)$ if and only if $\rho\left({ }^{x} g\right)=\sigma\left({ }^{y} 1\right)=$ 1 , so $c_{x}: \operatorname{Ker} \beta \rightarrow \operatorname{Ker} \rho$ is an isomorphism. Hence $c_{x}$ induces an isomorphism $\theta: H \rightarrow V$, which is immediately seen to be the same as the isomorphism induced by $c_{y}$. This completes the proof.

To see now why the definition of the functor $F$ (given the axiomatically defined $M$ ), is independent of choices we observe that in an expression $\Omega=\sum_{i=1}^{t} \lambda_{i} \Omega_{i}$ the $\lambda_{i} \in R$ are determined, and the $\Omega_{i}$ are determined up to isomorphism. We thus need to see that if we write $\Omega_{i}$ in two isomorphic ways, in the manner of the lemma, then 
$\alpha_{*} \beta^{*} \gamma_{*} \delta^{*}=\pi_{*} \rho^{*} \sigma_{*} \tau^{*}$. But now using the axioms for $M$ we have

$$
\begin{aligned}
\alpha_{*} \beta^{*} \gamma_{*} \delta^{*} & =\alpha_{*} \beta^{*} \theta^{*} \theta_{*} \gamma_{*} \delta^{*} \\
& =\alpha_{*}(\theta \beta)^{*}(\theta \gamma)_{*} \delta^{*} \\
& =\alpha_{*}\left(\rho c_{g}\right)^{*}\left(\sigma c_{h}\right)_{*} \delta^{*} \\
& =\alpha_{*} c_{g}^{*} \rho^{*} \sigma_{*} c_{h *} \delta^{*} \\
& =\alpha_{*} c_{g^{-1}} \rho^{*} \sigma_{*} c_{h^{-1}} \delta^{*} \\
& =\left(\alpha c_{g^{-1}}\right)_{*} \rho^{*} \sigma_{*}\left(\delta c_{h^{-1}}\right)^{*} \\
& =\left(c_{g^{-1}} \pi\right)_{*} \rho^{*} \sigma_{*}\left(c_{h^{-1}} \tau\right)^{*} \\
& =c_{g^{-1}} \pi_{*} \rho^{*} \sigma_{*} \tau^{*} c_{h^{-1}} \\
& =\pi_{*} \rho^{*} \sigma_{*} \tau^{*}
\end{aligned}
$$

as required.

We need also to see that $F$ is a functor, namely $F(\Omega \circ \Psi)=F(\Omega) F(\Psi)$ always holds. To see this, recall that we first defined $F$ on special bisets of the form ${ }_{G} K_{K}$ and ${ }_{K} K_{G}$ where the morphism $G \rightarrow K$ is either an inclusion of subgroups or a surjection. We then used Bouc's Lemma 2.1 to extend the definition to all morphisms by composition. We thus see that $F$ will be a functor precisely if it satisfies $F(\Omega \circ \Psi)=F(\Omega) F(\Psi)$ when $\Omega$ and $\Psi$ are taken to be special bisets. Since there are four kinds of special biset, depending on whether $G$ acts from the left or the right, and whether the homomorphism is an inclusion or a surjection, this gives $4 \cdot 4=16$ different types of composite which $F$ should preserve.

For each of these 16 composites $\Omega \circ \Psi$ when $\Omega$ and $\Psi$ are special, either the composite is already a product of the kind prescribed by Bouc's Lemma 2.1 (by adjoining bisets of the form ${ }_{G} G_{G}$ as necessary one may extend the product of length 2 to a product of length 4 ), or else it may be expressed as a linear combination of products of that kind. In the former case, the definition of $F$ guarantees that $F(\Omega \circ \Psi)=F(\Omega) F(\Psi)$. In the latter case, if $\Omega \circ \Psi=\sum_{i=1}^{t} \lambda_{i} \Omega_{i}$ where the $\Omega_{i}$ are transitive, the definition of $F(\Omega \circ \Psi)$ is $\sum_{i=1}^{t} \lambda_{i} F\left(\Omega_{i}\right)$, and $F$ will preserve composites precisely if $F(\Omega) \circ F(\Psi)=\sum_{i=1}^{t} \lambda_{i} F\left(\Omega_{i}\right)$. In each of the 16 cases, one of the axioms which $M$ satisfies implies this equality. We summarize which axiom must be used in Table 1, leaving the entry blank when the composite is already one of the products in the form of Lemma 2.1. This completes the proof that an object $M$ satisfying the axioms $(1)-(5)$ gives rise to a functor $F$ in $\operatorname{Mack}_{R}^{\mathcal{X}, \mathcal{Y}}(\mathcal{D})$ in the way we have described.

We conclude this section of definitions and basic results by mentioning the contravariant functor $*: \operatorname{Mack}_{R}^{\mathcal{X}, \mathcal{Y}} \rightarrow \operatorname{Mack}_{R}^{\mathcal{Y}, \mathcal{X}}$ which is a 
TABLE 1. Axioms for the decomposition of products of special bisets $\Omega \circ \Psi$.

\begin{tabular}{|c|c|c|c|c|c|}
\hline & \multicolumn{4}{|l|}{$\Psi$} \\
\hline & & \multicolumn{2}{|c|}{$\begin{array}{l}\qquad \Psi={ }_{K} K_{G} \\
\text { inclusion } \quad \text { epi }\end{array}$} & \multicolumn{2}{|c|}{$\begin{array}{l}\Psi={ }_{K} G_{G} \\
\text { inclusion } \quad \text { epi }\end{array}$} \\
\hline \multirow[t]{4}{*}{$\Omega$} & \multirow{2}{*}{$\begin{array}{r}\Omega={ }_{G} G_{K} \text { inclusion } \\
\text { epi }\end{array}$} & Axiom 1 & & & \\
\hline & & Axiom 1 & Axiom 1 & & Axiom 4 \\
\hline & \multirow{2}{*}{$\begin{array}{rr}\Omega={ }_{G} K_{K} \text { inclusion } \\
\text { epi }\end{array}$} & Axiom 5 & Axiom 3 & Axiom 1 & Axiom 1 \\
\hline & & Axiom 3 & & & Axiom 1 \\
\hline
\end{tabular}

duality between the full subcategories of $\operatorname{Mack}_{R}^{\mathcal{X}, \mathcal{Y}}$ and $\operatorname{Mack}_{R}^{\mathcal{Y}, \mathcal{X}}$ whose objects are functors $M$ for which every $M(G)$ is a finitely generated projective $R$-module. For each functor $M$ in $\operatorname{Mack}_{R}^{\mathcal{X}, \mathcal{Y}}$ we put $M^{*}(G)=$ $\operatorname{Hom}_{R}(M(G), R)$. For each $(H, G)$-biset ${ }_{H} \Omega_{G}$ there is an opposite $(G, H)$ biset ${ }_{G}\left(\Omega^{\mathrm{op}}\right)_{H}$ which has the same underlying set as $\Omega$ and where the left and right actions of $G$ and $H$ are obtained from $\Omega$ via the antiautomorphisms $g \mapsto g^{-1}$ and $h \mapsto h^{-1}$ of $G$ and $H$. Now the morphism $M^{*}\left({ }_{H} \Omega_{G}\right): M^{*}(G) \rightarrow M^{*}(H)$ is defined to be $M\left({ }_{G}\left(\Omega^{\mathrm{op}}\right)_{H}\right)^{*}$.

\section{Restriction, induCtion AND THE VERTEX}

In this section we study the restriction and induction of globally defined Mackey functors between different domains of definition. We describe the effect on simple and projective functors, and conclude with a theory of vertices of globally defined Mackey functors analogous in spirit to the theory of vertices and sources in group representation theory. There are some differences between the two theories: we will show that induction always sends indecomposables to indecomposables; and if an indecomposable functor is projective relative to a set of groups, its restriction to those groups is again indecomposable. A feature of these results is that we do not need to put any special hypothesis on the commutative ring $R$ : we do not invoke the Krull-Schmidt theorem and the theory works perfectly well over $\mathbb{Z}$, for example.

Suppose we have two section-closed sets of finite groups $\mathcal{E} \subseteq \mathcal{D}$ and consider the categories of globally defined Mackey functors defined on them. The restriction functor $\operatorname{Mack}_{R}^{\mathcal{X}, \mathcal{Y}}(\mathcal{D}) \rightarrow \operatorname{Mack}_{R}^{\mathcal{X}, \mathcal{Y}}(\mathcal{E})$ is given by restricting the domain of definition of a functor $M$ from $\mathcal{D}$ to $\mathcal{E}$. We will 
write $M \downarrow_{\mathcal{E}}^{\mathcal{D}}$ for this restricted functor. Restriction is an exact functor, because exactness of a sequence of globally defined Mackey functors is determined by exactness at each evaluation on a group. It has a left adjoint $N \mapsto N \uparrow_{\mathcal{E}}^{\mathcal{D}}=\mu_{R}^{\mathcal{X}, \mathcal{Y}}(\mathcal{D}) \otimes_{\mu_{R}^{\mathcal{X}, \mathcal{Y}}(\mathcal{E})} N$ called induction and a right adjoint $N \mapsto M \Uparrow_{\mathcal{E}}^{\mathcal{D}}=\operatorname{Hom}_{\mu_{R}^{\mathcal{X}, \mathcal{Y}}(\mathcal{E})}\left(\mu_{R}^{\mathcal{X}, \mathcal{Y}}(\mathcal{D}), N\right)$ called coinduction.

Regarding $M$ as a $\mu_{R}^{\mathcal{X}, \mathcal{Y}}(\mathcal{D})$-module we can express restriction to $\mathcal{E}$ in a way which is familiar from other areas of representation theory, at least when $\mathcal{E}$ is finite. In that case we have an idempotent $e_{\mathcal{E}}=$ $\sum_{G \in \mathcal{E} G} G_{G}$ and it is immediate from the definitions that $\mu_{R}^{\mathcal{X}, \mathcal{Y}}(\mathcal{E})=$ $e_{\mathcal{E}} \mu_{R}^{\mathcal{X}, \mathcal{Y}}(\mathcal{D}) e_{\mathcal{E}}$ and that regarding $M$ as a $\mu_{R}^{\mathcal{X}, \mathcal{Y}}(\mathcal{D})$-module its restriction is $e_{\mathcal{E}} M$. This is a functor which is described in [23, 6.2] as well as elsewhere, and the general properties hold here.

We start by recording the effect of restriction on the simple globally defined Mackey functors. Recall from [7] that the simple globally defined Mackey functors on a section-closed set of finite groups $\mathcal{D}$ are parametrized by pairs $(H, V)$ where $H$ is a group in $\mathcal{D}$ and $V$ is a simple $R$ Out $H$-module. For the moment we will write $S_{H, V}^{\mathcal{D}}$ for the corresponding simple functor defined on $\mathcal{D}$, but later we will omit $\mathcal{D}$ from the notation. It is characterized among simple functors by being non-zero only on groups which have $H$ as a section, and by the fact that at $H$ it is $S_{H, V}^{\mathcal{D}}(H)=V$.

Proposition 3.1. Let $\mathcal{E} \subseteq \mathcal{D}$ be sets of finite groups closed under taking sections. Then

$$
S_{H, V}^{\mathcal{D}} \downarrow_{\mathcal{E}}= \begin{cases}S_{H, V}^{\mathcal{E}} & \text { if } H \in \mathcal{E} \\ 0 & \text { if } H \notin \mathcal{E}\end{cases}
$$

Proof. This is immediate from Bouc's construction of the simple functors in [7], in that the value of a simple functor at a group $H$ is independent of the section-closed set of groups containing $H$.

Thus the restriction of a simple functor is either simple or zero, and a complete set of simple functors on the smaller set $\mathcal{E}$ arises via restriction, something which we know in generality from [23, 6.2g], but here we can also say that restriction preserves the parametrization of simple functors.

We are about to develop the theory of induction and restriction between different section-closed sets of finite groups and take inspiration from the corresponding theory for group representations. We will find that in several places the theory here is simpler, and stronger results hold, than for group representations. The first example of this is seen as part (3) of the next result. 
Proposition 3.2. Let $\mathcal{E} \subseteq \mathcal{D}$ be sets of finite groups closed under taking sections. Suppose $M$ is a globally defined Mackey functor defined on $\mathcal{E}$. Then

(1) $M \uparrow_{\mathcal{E}}^{\mathcal{D}}$ is generated by its values on groups in $\mathcal{E}$.

(2) $M \uparrow_{\mathcal{E}}^{\mathcal{D}} \downarrow_{\mathcal{E}}^{\mathcal{D}} \cong M$, and

(3) if $M$ is indecomposable then $M \uparrow_{\mathcal{E}}^{\mathcal{D}}$ is indecomposable.

Proof. (1) This comes directly from the definition of $M \uparrow_{\mathcal{E}}^{\mathcal{D}}$ as a tensor product.

(2) From the definition of the global Mackey algebra, when we regard $\mu_{R}^{\mathcal{X}, \mathcal{Y}}(\mathcal{D})$ as a $\left(\mu_{R}^{\mathcal{X}, \mathcal{Y}}(\mathcal{E}), \mu_{R}^{\mathcal{X}, \mathcal{Y}}(\mathcal{E})\right)$-bimodule it is isomorphic to the direct sum of $\mu_{R}^{\mathcal{X}, \mathcal{Y}}(\mathcal{E})$ and a summand on which the action is zero, and so

$$
M \uparrow_{\mathcal{E}}^{\mathcal{D}} \downarrow_{\mathcal{E}}^{\mathcal{D}}=\left(\mu_{R}^{\mathcal{X}, \mathcal{Y}}(\mathcal{D}) \otimes_{\mu_{R}^{\mathcal{X}, \mathcal{Y}}(\mathcal{E})} M\right) \downarrow_{\mathcal{E}}^{\mathcal{D}} \cong \mu_{R}^{\mathcal{X}, \mathcal{Y}}(\mathcal{E}) \otimes_{\mu_{R}^{\mathcal{X}, \mathcal{Y}}(\mathcal{E})} M \cong M
$$

as left $\mu_{R}^{\mathcal{X}, \mathcal{Y}}(\mathcal{E})$-modules.

(3) The induced functor $M \uparrow_{\mathcal{E}}^{\mathcal{D}}$ is generated by its values on $\mathcal{E}$. Thus if $M \uparrow_{\mathcal{E}}^{\mathcal{D}}=M_{1} \oplus M_{2}$ is a direct sum decomposition then $M_{1}$ and $M_{2}$ are also be generated by their values on $\mathcal{E}$. Hence if both $M_{1}$ and $M_{2}$ are non-zero, they must be non-zero on $\mathcal{E}$. In this case $M \cong M \uparrow_{\mathcal{E}}^{\mathcal{D}} \downarrow_{\mathcal{E}}^{\mathcal{D}}=$ $M_{1} \downarrow_{\mathcal{E}}^{\mathcal{D}} \oplus M_{2} \downarrow_{\mathcal{E}}^{\mathcal{D}}$ is a non-trivial decomposition of $M$, which is not possible. Thus one of $M_{1}$ and $M_{2}$ must be zero.

In [32] and [7] it is shown that when $R$ is a field or a discrete valuation ring the simple functor $S_{H, V}$ has a projective cover $P_{H, V}$, and it is a summand of the representable functor $\operatorname{Hom}_{\mathcal{C}_{R}^{\mathcal{X}}, \mathcal{Y}}(G, \quad)$ with multiplicity equal to $\operatorname{dim} S_{H, V}(G) / \operatorname{dim} \operatorname{End}_{R \text { Out } H}(V)$. We observe that these indecomposable projective functors behave well under restriction and induction.

Proposition 3.3. Let $\mathcal{E} \subseteq \mathcal{D}$ be sets of finite groups closed under taking sections, suppose that $R$ is a field or a discrete valuation ring, and let $H$ be a group in $\mathcal{E}$. Let $P_{H, V}^{\mathcal{E}}$ and $P_{H, V}^{\mathcal{D}}$ be the projective covers of the simple functors $S_{H, V}^{\mathcal{E}}$ and $S_{H, V}^{\mathcal{D}}$ as functors defined on $\mathcal{E}$ and $\mathcal{D}$, respectively. Then $P_{H, V}^{\mathcal{D}} \downarrow_{\mathcal{E}}^{\mathcal{D}}=P_{H, V}^{\mathcal{E}}$ and $P_{H, V}^{\mathcal{E}} \uparrow_{\mathcal{E}}^{\mathcal{D}}=P_{H, V}^{\mathcal{D}}$.

Proof. Induction from $\mathcal{E}$ to $\mathcal{D}$ sends projectives to projectives (since it is left adjoint to an exact functor) and preserves indecomposability (by Proposition $3.1(3)$ ), so $P_{H, V}^{\mathcal{E}} \uparrow_{\mathcal{E}}^{\mathcal{D}}$ is an indecomposable projective functor. Since $S_{H, V}^{\mathcal{D}} \downarrow_{\mathcal{E}}=S_{H, V}^{\mathcal{E}}$, by adjointness there is a non-zero morphism $S_{H, V}^{\mathcal{E}} \uparrow_{\mathcal{E}}^{\mathcal{D}} \rightarrow S_{H, V}^{\mathcal{D}}$, which must be a surjection. There is also a surjection $P_{H, V}^{\mathcal{E}} \uparrow_{\mathcal{E}}^{\mathcal{D}} \rightarrow S_{H, V}^{\mathcal{E}} \uparrow_{\mathcal{E}}^{\mathcal{D}}$, and hence $S_{H, V}^{\mathcal{D}}$ is a homomorphic image of $P_{H, V}^{\mathcal{E}} \uparrow_{\mathcal{E}}^{\mathcal{D}}$. It follows that $P_{H, V}^{\mathcal{E}} \uparrow_{\mathcal{E}}^{\mathcal{D}}=P_{H, V}^{\mathcal{D}}$.

Finally, $P_{H, V}^{\mathcal{E}}=P_{H, V}^{\mathcal{E}} \uparrow_{\mathcal{E}}^{\mathcal{D}} \downarrow_{\mathcal{E}}^{\mathcal{D}}=P_{H, V}^{\mathcal{D}} \downarrow_{\mathcal{E}}^{\mathcal{D}}$, by $3.2(2)$. 
The above ideas may be developed into a theory of relative projectivity of globally defined Mackey functors. In the context of section-closed sets of groups $\mathcal{E} \subseteq \mathcal{D}$ we will say that a functor $M$ defined on $\mathcal{D}$ is projective relative to $\mathcal{E}$ (or $\mathcal{E}$-projective) if and only if the canonical counit homomorphism $M \downarrow_{\mathcal{E}}^{\mathcal{D}} \uparrow_{\mathcal{E}}^{\mathcal{D}} \rightarrow M$ is a split epimorphism. This condition is equivalent to a number of other conditions, and many of these are listed in [3, Prop. VI.3.6] in a context which does not have to do with group representations. Thus $M$ is $\mathcal{E}$-projective if and only if $M$ is a direct summand of $M \downarrow_{\mathcal{E}}^{\mathcal{D}} \uparrow_{\mathcal{E}}^{\mathcal{D}}$, which happens if and only if $M$ is a direct summand of $N \uparrow_{\mathcal{E}}^{\mathcal{D}}$ for some functor $N$ defined on $\mathcal{E}$. (The argument which proves this is standard, and given in [2] in a way which does not depend on the properties of group representations.) In fact the situation we are now considering is less delicate than this as we see in the next proposition, which is a strengthening of a result which in a slightly different context is due to $\mathrm{Xu}$ [38]. Part (2) of this result is a partial converse to Proposition 3.2(3).

Proposition 3.4. Let $\mathcal{E} \subseteq \mathcal{D}$ be sets of finite groups closed under taking sections and let $M$ be a globally defined Mackey functor defined on $\mathcal{D}$.

(1) If $M$ is $\mathcal{E}$-projective then the counit homomorphism $M \downarrow_{\mathcal{E}}^{\mathcal{D}} \uparrow_{\mathcal{E}}^{\mathcal{D}} \rightarrow$ $M$ is an isomorphism, so that $M \cong M \downarrow_{\mathcal{E}}^{\mathcal{D}} \uparrow_{\mathcal{E}}^{\mathcal{D}}$.

(2) If $M$ is $\mathcal{E}$-projective and indecomposable then $M \downarrow_{\mathcal{E}}^{\mathcal{D}}$ is also indecomposable.

Proof. (1) The hypothesis is that the counit homomorphism is split. On evaluation at groups $G$ in $\mathcal{E}$ this homomorphism is an isomorphism, so that a splitting provides a decomposition $M \downarrow_{\mathcal{E}}^{\mathcal{D}} \uparrow_{\mathcal{E}}^{\mathcal{D}} \cong M \oplus M^{\prime}$ where $M^{\prime}(G)=0$ for all $G$ in $\mathcal{E}$. Now $M \downarrow_{\mathcal{E}}^{\mathcal{D}} \uparrow_{\mathcal{E}}^{\mathcal{D}}$ is generated by its values on $G$ in $\mathcal{E}$, and hence so is $M^{\prime}$ since it is an image of $M$. It follows that $M^{\prime}=0$ and the counit is an isomorphism.

(2) If $M \downarrow_{\mathcal{E}}^{\mathcal{D}}=N_{1} \oplus N_{2}$ decomposes then

$$
M \cong M \downarrow_{\mathcal{E}}^{\mathcal{D}} \uparrow_{\mathcal{E}}^{\mathcal{D}}=N_{1} \uparrow_{\mathcal{E}}^{\mathcal{D}} \oplus N_{2} \uparrow_{\mathcal{E}}^{\mathcal{D}}
$$

also decomposes, and if $M$ is indecomposable this cannot happen.

Corollary 3.5. Let $\mathcal{E} \subseteq \mathcal{D}$ be sets of finite groups closed under taking sections, let $M$ be a globally defined Mackey functor defined on $\mathcal{D}$ and let $N$ be a globally defined Mackey functor defined on $\mathcal{E}$. Suppose that $M$ and $N$ are indecomposable.

(1) If $M$ is a summand of $N \uparrow_{\mathcal{E}}^{\mathcal{D}}$ then $N \cong M \downarrow_{\mathcal{E}}^{\mathcal{D}}$.

(2) If $M$ is projective relative to $\mathcal{E}$ and $N$ is a summand of $M \downarrow_{\mathcal{E}}^{\mathcal{D}}$ then $M \cong N \uparrow_{\mathcal{E}}^{\mathcal{D}}$. 
Proof. (1) By Proposition $3.2(3), N \uparrow_{\mathcal{E}}^{\mathcal{D}}$ is indecomposable, so that $M \cong N \uparrow_{\mathcal{E}}^{\mathcal{D}}$ and $N \cong N \uparrow_{\mathcal{E}}^{\mathcal{D}} \downarrow_{\mathcal{E}}^{\mathcal{D}} \cong M \downarrow_{\mathcal{E}}^{\mathcal{D}}$ by $3.2(2)$.

(2) By Proposition 3.4(2) we see that $M \downarrow_{\mathcal{E}}^{\mathcal{D}}$ is indecomposable and hence equal to $N$. The statement follows from Proposition 3.4(1).

We will show that there is always a unique minimal section-closed set of finite groups relative to which an indecomposable functor is projective. This will follow from the next result, for which we introduce some notation. We suppose that $\mathcal{D}$ and $\mathcal{E}$ are sets of finite groups closed under taking sections, but do not assume this time that $\mathcal{E} \subseteq \mathcal{D}$. Let us write ${ }_{\mathcal{D}} \mu_{\mathcal{E}}=\bigoplus_{\substack{G \in \mathcal{D} \\ H \in \mathcal{E}}} A_{R}^{\mathcal{X}, \mathcal{Y}}(G, H)$. This is an $R$-submodule of $\mu_{R}^{\mathcal{X}, \mathcal{Y}}$ and it is a $\left(\mu_{R}^{\mathcal{X}, \mathcal{Y}}(\mathcal{D}), \mu_{R}^{\mathcal{X}, \mathcal{Y}}(\mathcal{E})\right)$-bimodule. Its significance is that if $\mathcal{G}$ is a further set of finite groups closed under taking sections which contains both $\mathcal{D}$ and $\mathcal{E}$ then $M \uparrow_{\mathcal{E}}^{\mathcal{G}} \downarrow_{\mathcal{D}}^{\mathcal{G}} \cong{ }_{\mathcal{D}} \mu_{\mathcal{E}} \otimes_{\mu_{R}^{\mathcal{X}}, \mathcal{Y}}(\mathcal{E})$

Proposition 3.6. Let $\mathcal{D}$ and $\mathcal{E}$ be sets of finite groups closed under taking sections and let $\mathcal{D} \cap \mathcal{E}$ denote a set of groups whose isomorphism types are exactly those in both $\mathcal{D}$ and $\mathcal{E}$. Then multiplication induces an isomorphism

$$
\mathcal{D} \mu_{(\mathcal{D} \cap \mathcal{E})} \otimes_{\mu_{R}^{\mathcal{X}, \mathcal{Y}}(\mathcal{D} \cap \mathcal{E})}(\mathcal{D} \cap \mathcal{E}) \mu_{\mathcal{E}} \rightarrow{ }_{\mathcal{D}} \mu_{\mathcal{E}}
$$

Proof. From the definition $\mathcal{D} \mu_{\mathcal{E}}$ has as a basis the transitive $(G, H)$ bisets where $G \in \mathcal{D}$ and $H \in \mathcal{E}$. By Bouc's Lemma 2.1 these can all be written in the form $\left({ }_{G} G_{A} \circ{ }_{A} J_{J}\right) \circ\left({ }_{J} J_{B} \circ{ }_{B} H_{H}\right)$. Since necessarily $J \in \mathcal{D} \cap \mathcal{E}$ this shows that the multiplication map is surjective.

In what follows all tensor products are taken over $\mu_{R}^{\mathcal{X}, \mathcal{Y}}(\mathcal{D} \cap \mathcal{E})$, and we leave this out of the notation to simplify matters. Consider an element

$$
\sum_{G, J, K, H, i} \lambda_{G, J, K, H, i}\left({ }_{G} \Omega_{i J} \otimes_{K} \Psi_{i H}\right)
$$

which lies in the kernel of the multiplication map, so

$$
\sum_{G, J, K, H, i} \lambda_{G, J, K, H, i}\left({ }_{G} \Omega_{i J} \circ{ }_{K} \Psi_{i H}\right)=0 .
$$

We will show that the first sum with the tensor product is zero. Firstly we may assume in this sum that $J=K$ since the idempotent ${ }_{J} J_{J}$ lies in $\mu_{R}^{\mathcal{X}, \mathcal{Y}}(\mathcal{D} \cap \mathcal{E})$ and ${ }_{G} \Omega_{i J} \otimes_{K} \Psi_{i H}={ }_{G} \Omega_{i J}{ }_{J} J_{J} \otimes_{K} \Psi_{i H}={ }_{G} \Omega_{i J} \otimes_{J} J_{J}{ }_{K} \Psi_{i H}$. This is zero if $K \neq J$.

Next we may assume that $G \in \mathcal{D}$ and $H \in \mathcal{E}$ are fixed, since we may multiply all formulas on the left by ${ }_{G} G_{G}$ and on the right by ${ }_{H} H_{H}$ to 
get an element of the tensor product

$$
\sum_{J, i} \lambda_{G, J, J, H, i}\left({ }_{G} \Omega_{i J} \otimes{ }_{J} \Psi_{i H}\right)
$$

for which

$$
\sum_{J, i} \lambda_{G, J, J, H, i}\left({ }_{G} \Omega_{i J} \circ{ }_{J} \Psi_{i H}\right)=0 .
$$

We now claim that we may express each ${ }_{G} \Omega_{i J} \otimes{ }_{J} \Psi_{i H}$ as a linear combination of terms of the form

$$
\left({ }_{G} G_{A_{j}} \circ{ }_{A_{j}} J_{j_{j}}\right) \otimes\left({ }_{J_{j}} J_{j_{B_{j}}} \circ{ }_{B_{j}} H_{H}\right)
$$

where each of the four bisets is of the kind which appears in Bouc's Lemma 2.1. Simplifying the notation and omitting subscripts $i$ and $j$, we may assume that $\Omega$ and $\Psi$ are transitive and write $\Omega={ }_{G} G_{A}{ }^{\circ}{ }_{A} B_{B} \circ$ ${ }_{B} B_{C} \circ{ }_{C} J_{J}$ and $\Psi={ }_{J} J_{D} \circ{ }_{D} E_{E} \circ{ }_{E} E_{F} \circ{ }_{F} H_{H}$. Note that $B, C, J, D$ and $E$ are all groups which occur in $\mathcal{D} \cap \mathcal{E}$ since these groups are all sections of $J$. This means we can write

$$
\Omega \otimes \Psi=\left({ }_{G} G_{A} \circ{ }_{A} B_{B}\right) \otimes\left({ }_{B} B_{C} \circ{ }_{C} J_{J} \circ{ }_{J} J_{D} \circ{ }_{D} E_{E} \circ{ }_{E} E_{F} \circ{ }_{F} H_{H}\right)
$$

by passing the terms in $\mathcal{D} \cap \mathcal{E}$ across the tensor product. By Bouc's Lemma 2.1 we can express the term on the right of the tensor product as a sum of terms of the form ${ }_{B} B_{K} \circ{ }_{K} L_{L} \circ{ }_{L} L_{M} \circ{ }_{M} H_{H}$ where now $B$, $K$ and $L$ are groups which appear in $\mathcal{D} \cap \mathcal{E}$. Thus $\Omega \otimes \Psi$ is a sum of terms

$$
\begin{aligned}
\left({ }_{G} G_{A} \circ{ }_{A} B_{B}\right) \otimes & \left({ }_{B} B_{K} \circ{ }_{K} L_{L} \circ{ }_{L} L_{M} \circ{ }_{M} H_{H}\right) \\
& =\left({ }_{G} G_{A} \circ{ }_{A} B_{B} \circ{ }_{B} B_{K} \circ{ }_{K} L_{L}\right) \otimes\left({ }_{L} L_{M} \circ{ }_{M} H_{H}\right)
\end{aligned}
$$

where we have obtained the expression on the right of the equality by passing two terms across the tensor product. We now write the product of four terms on the left of the last tensor product as a sum of terms of the form ${ }_{G} G_{N} \circ{ }_{N} P_{P} \circ{ }_{P} P_{Q} \circ{ }_{Q} L_{L}$ where $P$ is a section of $L$, and so $\Omega \otimes \Psi$ is a sum of terms

$$
\begin{aligned}
\left({ }_{G} G_{N} \circ{ }_{N} P_{P} \circ{ }_{P} P_{Q} \circ{ }_{Q} L_{L}\right) \otimes\left({ }_{L} L_{M} \circ{ }_{M} H_{H}\right) \\
=\left({ }_{G} G_{N} \circ{ }_{N} P_{P}\right) \otimes\left({ }_{P} P_{Q} \circ{ }_{Q} L_{L} \circ{ }_{L} L_{M} \circ{ }_{M} H_{H}\right) .
\end{aligned}
$$

Again, we have obtained the expression on the right of the equality by passing two terms across the tensor product. Iterating this process where we pass two terms across the tensor product and rewrite the new product, at each stage the group adjacent to the tensor product is a section of the previous one. Thus the process must reach a stage where each group adjacent to the tensor product is isomorphic to the previous one, and when this happens the biset which is passed across the tensor product is a copy of that group acted upon regularly, except 
that the action may be twisted by an isomorphism. By incorporating such an isomorphism in the action on this group from the other side, we express each ${ }_{G} \Omega_{i J} \otimes{ }_{J} \Psi_{i H}$ as claimed.

We have now produced a linear combination of tensor products

$$
\left({ }_{G} G_{A_{j}} \circ A_{j} J_{j_{j}}\right) \otimes\left(J_{j} J_{j_{B_{j}}} \circ{ }_{B_{j}} H_{H}\right)
$$

in which each biset is special and such that the corresponding linear combination of products

$$
{ }_{G} G_{A_{j}} \circ{ }_{A_{j}} J_{j_{J_{j}}} \circ{ }_{J_{j}} J_{j_{B_{j}}} \circ{ }_{B_{j}} H_{H}
$$

is zero. But such expressions, taken up to isomorphism, are basis elements of $\mathcal{D} \mu_{\mathcal{E}}$, and so for each isomorphism type the sum of the coefficients of the corresponding tensor products is zero. Lemma 2.2 implies that if $G \circ J \circ J \circ H \cong G \circ J^{\prime} \circ J^{\prime} \circ H$ then $G \circ J \otimes J \circ H=G \circ J^{\prime} \otimes J^{\prime} \circ H$ and it follows that the linear combination of tensors we were considering must be zero.

Corollary 3.7. Let $\mathcal{E}_{1} \subseteq \mathcal{D}$ and $\mathcal{E}_{2} \subseteq \mathcal{D}$ be sets of finite groups which are closed under taking sections and let $M$ be a globally defined Mackey functor defined on $\mathcal{D}$ which is projective relative to $\mathcal{E}_{1}$ and also projective relative to $\mathcal{E}_{2}$. Then $M$ is projective relative to $\mathcal{E}_{1} \cap \mathcal{E}_{2}$.

Proof. We have $M \cong M \downarrow_{\mathcal{E}_{1}}^{\mathcal{D}} \uparrow_{\mathcal{E}_{1}}^{\mathcal{D}} \cong M \downarrow_{\mathcal{E}_{2}}^{\mathcal{D}} \uparrow_{\mathcal{E}_{2}}^{\mathcal{D}}$ so that $M \cong M \downarrow_{\mathcal{E}_{1}}^{\mathcal{D}} \uparrow{ }_{\mathcal{E}_{1}}^{\mathcal{D}} \downarrow_{\mathcal{E}_{2}}^{\mathcal{D}} \uparrow^{\mathcal{D}} \mathcal{E}_{\mathcal{E}_{2}}$. Now for any Mackey functor $N$ defined on $\mathcal{E}_{1}$ we have

$$
\begin{aligned}
& N \uparrow_{\mathcal{E}_{1} \downarrow_{\mathcal{E}_{2}}^{\mathcal{D}}}=\mu_{R}^{\mathcal{X}, \mathcal{Y}}(\mathcal{D}) \otimes_{\mu_{R}^{\mathcal{X}, \mathcal{Y}}\left(\mathcal{E}_{1}\right)} N \\
&=\mathcal{E}_{2} \mu_{\mathcal{E}_{1}} \otimes_{\mu_{R}^{\mathcal{X}, \mathcal{Y}}\left(\mathcal{E}_{1}\right)} N \\
& \cong \mathcal{E}_{2} \mu_{\left(\mathcal{E}_{2} \cap \mathcal{E}_{1}\right)} \otimes_{\mu_{R}^{\mathcal{X}, \mathcal{Y}}\left(\mathcal{E}_{2} \cap \mathcal{E}_{1}\right)}\left(\mathcal{E}_{2} \cap \mathcal{E}_{1}\right) \mu_{\mathcal{E}_{1}} \otimes_{\mu_{R}^{\mathcal{X}, \mathcal{Y}}\left(\mathcal{E}_{1}\right)} N \\
& \cong \mathcal{E}_{2} \mu_{\left(\mathcal{E}_{2} \cap \mathcal{E}_{1}\right)} \otimes_{\mu_{R}^{\mathcal{X}, \mathcal{Y}}\left(\mathcal{E}_{2} \cap \mathcal{E}_{1}\right)} N \downarrow_{\mathcal{E}_{1} \cap \mathcal{E}_{2}} \\
& \cong N \downarrow_{\mathcal{E}_{1} \cap \mathcal{E}_{1}} \uparrow_{\mathcal{E}_{1} \cap \mathcal{E}_{2}}^{\mathcal{E}_{2}}
\end{aligned}
$$

using 3.6. Thus $M \cong M \downarrow_{\mathcal{E}_{1}}^{\mathcal{D}} \downarrow_{\mathcal{E}_{1} \cap \mathcal{E}_{2}}^{\mathcal{E}_{1}} \uparrow_{\mathcal{E}_{1} \cap \mathcal{E}_{2}}^{\mathcal{E}_{2}} \uparrow_{\mathcal{E}_{2}}^{\mathcal{D}} \cong M \downarrow_{\mathcal{E}_{1} \cap \mathcal{E}_{2}}^{\mathcal{D}} \uparrow \uparrow_{\mathcal{E}_{1} \cap \mathcal{E}_{2}}^{\mathcal{D}}$ as required.

Corollary 3.8. Let $\mathcal{E}_{1}, \mathcal{E}_{2}, \mathcal{D}$ and $\mathcal{G}$ be sets of finite groups which are closed under taking sections with $\mathcal{E}_{1} \subseteq \mathcal{D}, \mathcal{E}_{2} \subseteq \mathcal{D}$ and $\mathcal{D} \subseteq \mathcal{G}$. Let $M$ be a globally defined Mackey functor defined on $\mathcal{D}$ which is projective relative to $\mathcal{E}_{1}$. Then

(1) $M \uparrow_{\mathcal{D}}^{\mathcal{G}}$ is projective relative to $\mathcal{E}_{1}$, and

(2) $M \downarrow_{\mathcal{E}_{2}}^{\mathcal{D}}$ is projective relative to $\mathcal{E}_{1} \cap \mathcal{E}_{2}$.

Proof. The first statement is immediate from the transitivity of induction. For the second statement, suppose that $M$ is a summand of 
$N \uparrow \uparrow_{\mathcal{E}_{1}}^{\mathcal{D}}$. Then $M \downarrow_{\mathcal{E}_{2}}^{\mathcal{D}}$ is a summand of $N \uparrow_{\mathcal{E}_{1}}^{\mathcal{D}} \downarrow_{\mathcal{E}_{2}}^{\mathcal{D}}$, which is isomorphic to $N \downarrow_{\mathcal{E}_{1} \cap \mathcal{E}_{2}}^{\mathcal{E}_{1}} \uparrow_{\mathcal{E}_{1} \cap \mathcal{E}_{2}}^{\mathcal{E}_{2}}$ as in the last result and hence $M \downarrow_{\mathcal{E}_{2}}^{\mathcal{D}}$ is projective relative to $\mathcal{E}_{1} \cap \mathcal{E}_{2}$.

Theorem 3.9. Let $M$ be a globally defined Mackey functor. There is a unique minimal set of groups $\mathcal{E}$, closed under taking sections, relative to which $M$ is projective. Furthermore $M \cong M \downarrow_{\mathcal{E}}^{\mathcal{D}} \uparrow_{\mathcal{E}}^{\mathcal{D}}$, and $M \downarrow_{\mathcal{E}}^{\mathcal{D}}$ is (up to isomorphism) the only Mackey functor $N$ defined on $\mathcal{E}$ with the property that $M \cong N \uparrow_{\mathcal{E}}^{\mathcal{D}}$.

Proof. From the last result we see that $\mathcal{E}$ is the intersection of the sets of groups (closed under taking sections) relative to which $M$ is projective. If $M \cong N \uparrow_{\mathcal{E}}^{\mathcal{D}}$ then $N \cong N \uparrow_{\mathcal{E}}^{\mathcal{D}} \downarrow_{\mathcal{E}}^{\mathcal{D}}=M \downarrow_{\mathcal{E}}^{\mathcal{D}}$ determines $N$ uniquely.

We may call the set of groups $\mathcal{E}$ in the above theorem the vertex of the globally defined Mackey functor $M$.

Proposition 3.10. Let $H$ be a group and $V$ a simple $R \operatorname{Out}(H)$ module. The representable functor $\operatorname{Hom}_{\mathcal{C}_{R}^{\mathcal{X}, \mathcal{Y}}(\mathcal{D})}(H, \quad)$ and the indecomposable projective functor $P_{H, V}$ (assuming $R$ is a field or a discrete valuation ring) both have vertex the set of sections of $H$.

Proof. Let $\mathcal{E}$ be the set of sections of $H$. As a $\mu_{R}^{\mathcal{X}, \mathcal{Y}}(\mathcal{D})$-module the representable functor is

$$
\mu_{R}^{\mathcal{X}, \mathcal{Y}}(\mathcal{D}) \cdot{ }_{H} H_{H}=\mu_{R}^{\mathcal{X}, \mathcal{Y}}(\mathcal{D}) \otimes_{\mu_{R}^{\mathcal{X}, \mathcal{Y}}(\mathcal{E})} \mu_{R}^{\mathcal{X}, \mathcal{Y}}(\mathcal{E}) \cdot{ }_{H} H_{H}
$$

so that this functor is projective relative to $\mathcal{E}$. The vertex cannot be any smaller, since the functor is generated by its value at $H$, and not by its values on smaller groups.

We have seen in Proposition 3.3 that $P_{H, V}$ is projective relative to $\mathcal{E}$, and again it is not projective relative to any smaller set of groups, because it is not generated by its values on such groups.

\section{Filtrations, Brauer quotients and Restriction Kernels}

In this section we describe an ascending filtration and a descending filtration of each globally defined Mackey functor associated to an ordering of isomorphism types of finite groups. When we show in Section 7 that globally defined Mackey functors form a highest weight category over a field of characteristic zero, we will see that the ascending filtration of each projective functor refines to a filtration with standard factors $\Delta$, and the descending filtration of each injective functor refines to a filtration with costandard factors $\nabla$. Prior to that application we develop the properties of these filtrations, relating them to the Brauer quotient and restriction kernel constructions. 
Suppose that $\mathcal{E}$ is a set of finite groups. We define

$$
I_{\mathcal{E}} M=\langle M(J) \mid J \in \mathcal{E}\rangle
$$

to be the subfunctor of $M$ generated by its values on groups in $\mathcal{E}$. Thus $I_{\mathcal{E}} M$ is the smallest subfunctor of $M$ whose value on all the groups $J \in \mathcal{E}$ is $M(J)$.

It is useful to speak of a globally defined Mackey functor $M$ being cogenerated by subsets $X_{H} \subseteq M(H)$ where $H$ ranges over finite groups. By this we mean that every morphism of globally defined Mackey functors $M \rightarrow M^{\prime}$ which is injective on all the subsets $X_{H}$ is necessarily a monomorphism. Given a set of finite groups $\mathcal{E}$ we define $R_{\mathcal{E}} M$ to be the largest subfunctor of $M$ satisfying the condition that $M(\gamma): R_{\mathcal{E}} M(K) \rightarrow M(J)$ is the zero map for every morphism $\gamma: K \rightarrow J$ in $\mathcal{C}_{R}^{\mathcal{X}, \mathcal{Y}}$ with $J \in \mathcal{E}$. We see that $M / R_{\mathcal{E}} M$ takes the same values as $M$ on groups in $\mathcal{E}$, and is cogenerated by these values. We may call it the quotient of $M$ cogenerated by its values on groups in $\mathcal{E}$.

In the next proposition we write 'all' to denote a set of representatives of all isomorphism classes of finite groups.

Proposition 4.1. Let $M$ be a globally defined Mackey functor and let $\mathcal{E}, \mathcal{E}_{1}$ and $\mathcal{E}_{2}$ be sets of groups with $\mathcal{E}_{1} \subseteq \mathcal{E}_{2}$.

(1) $I_{\emptyset} M=R_{\text {all }} M=0$ and $R_{\emptyset} M=I_{\text {all }} M=M$.

(2) $I_{\mathcal{E}_{1}} M \subseteq I_{\mathcal{E}_{2}} M$ and $R_{\mathcal{E}_{1}} M \supseteq R_{\mathcal{E}_{2}} M$.

(3) If $\mathcal{E}$ is closed under taking sections then

$$
I_{\mathcal{E}} M(K)=\sum_{\substack{\gamma: G \rightarrow K \\ G \in \mathcal{E}}} \operatorname{Im} M(\gamma) \quad \text { and } \quad R_{\mathcal{E}} M(K)=\bigcap_{\substack{\gamma: K \rightarrow \mathcal{E} \\ G \in \mathcal{E}}} \operatorname{Ker} M(\gamma) \text {. }
$$

(4) If $\mathcal{E}_{1} \subseteq \mathcal{E}_{2}$ then $I_{\mathcal{E}_{2}} M / I_{\mathcal{E}_{1}} M$ is generated by its values on the subgroups in $\mathcal{E}_{2}-\mathcal{E}_{1}$ and $R_{\mathcal{E}_{1}} M / R_{\mathcal{E}_{2}} M$ is cogenerated by its values on the subgroups in $\mathcal{E}_{2}-\mathcal{E}_{1}$. If additionally $\mathcal{E}_{1}$ and $\mathcal{E}_{2}$ are closed under taking sections then $I_{\mathcal{E}_{2}} M / I_{\mathcal{E}_{1}} M$ and $R_{\mathcal{E}_{1}} M / R_{\mathcal{E}_{2}} M$ are zero on groups which do not contain a group in $\mathcal{E}_{2}-\mathcal{E}_{1}$ as a section.

(5) $I_{\mathcal{E}}\left(M_{1} \oplus M_{2}\right)=I_{\mathcal{E}} M_{1} \oplus I_{\mathcal{E}} M_{2}$ and $R_{\mathcal{E}}\left(M_{1} \oplus M_{2}\right)=R_{\mathcal{E}} M_{1} \oplus R_{\mathcal{E}} M_{2}$.

Proof. (1) and (2) are self-evident.

(3) The right hand side of the expression for $I_{\mathcal{E}} M(K)$ equals the subfunctor of $M$ generated by its values on groups in $\mathcal{E}$, since the right side is certainly contained in this subfunctor, and it is a subfunctor itself since on applying any morphism in $\mathcal{C}_{R}^{\mathcal{X}, \mathcal{Y}}(\mathcal{D})$ by functoriality of $M$ to a term on the right, the result factors through another of the terms on the right, by Bouc's Lemma 2.1 and using the hypothesis that $\mathcal{E}$ is section-closed. A similar argument applies to the expression 
for $R_{\mathcal{E}} M(K)$. Everything on the right goes to zero under morphisms $M(\gamma)$ where the codomain of $\gamma$ lies in $\mathcal{E}$, but we need to check that the expression on the right does define a subfunctor. This again follows from Bouc's Lemma.

In (4), we have from the definitions that $I_{\mathcal{E}_{2}} M$ is generated by $I_{\mathcal{E}_{1}} M$ and the values of $M$ on the groups in $\mathcal{E}_{2}-\mathcal{E}_{1}$. Hence $I_{\mathcal{E}_{2}} M / I_{\mathcal{E}_{1}} M$ is generated by its values on the subgroups in $\mathcal{E}_{2}-\mathcal{E}_{1}$ alone. The statement about cogeneration is dual. Suppose now that $\mathcal{E}_{1}$ and $\mathcal{E}_{2}$ are closed under taking sections. If all sections of a group which lie in $\mathcal{E}_{2}$ lie in $\mathcal{E}_{1}$ then $I_{\mathcal{E}_{2}} M$ and $I_{\mathcal{E}_{1}} M$ take the same values on this group by (3), and so the quotient functor is zero on such a group. Thus the quotient functor is only non-zero on groups which contain a section in $\mathcal{E}_{2}-\mathcal{E}_{1}$. Again the statement about $R_{\mathcal{E}_{1}} M / R_{\mathcal{E}_{2}} M$ is dual.

(5) is immediate.

In the next result we assume that $R$ is a field and show that the subfunctors $I_{\mathcal{E}} M$ and $R_{\mathcal{E}} M$ are dual to each other, in the same way as in [34, Prop. 2.2]. We recall from Section 2 that for each functor $M$ in $\operatorname{Mack}_{R}^{\mathcal{X}, \mathcal{Y}}$ there is a dual functor $M^{*}$ in $\operatorname{Mack}_{R}^{\mathcal{Y}, \mathcal{X}}$ with $M^{*}(G)=$ $\operatorname{Hom}_{R}(M(G), R)$. If $N \subseteq M$ is a subfunctor, let us put $N^{\perp}(K)=\{f \in$ $\left.M^{*}(K)|f|_{N(K)}=0\right\}$. Then by a standard piece of linear algebra if $N_{1} \subseteq N_{2} \subseteq M$ we have $\left(N_{2} / N_{1}\right)^{*} \cong N_{1}^{\perp} / N_{2}^{\perp}$.

Proposition 4.2. Let $M$ be a globally defined Mackey functor over a field $R$ and let $\mathcal{E}, \mathcal{E}_{1}$ and $\mathcal{E}_{2}$ be sets of finite groups closed under taking sections. Then $\left(I_{\mathcal{E}} M\right)^{\perp}=R_{\mathcal{E}}\left(M^{*}\right)$ and $\left(R_{\mathcal{E}} M\right)^{\perp}=I_{\mathcal{E}}\left(M^{*}\right)$. Thus if $\mathcal{E}_{1} \subseteq \mathcal{E}_{2}$ we have

$$
\left(I_{\mathcal{E}_{2}} M / I_{\mathcal{E}_{1}} M\right)^{*} \cong\left(I_{\mathcal{E}_{1}} M\right)^{\perp} /\left(I_{\mathcal{E}_{2}} M\right)^{\perp}=R_{\mathcal{E}_{1}}\left(M^{*}\right) / R_{\mathcal{E}_{2}}\left(M^{*}\right)
$$

and

$$
\left(R_{\mathcal{E}_{1}} M / R_{\mathcal{E}_{2}} M\right)^{*} \cong\left(R_{\mathcal{E}_{2}} M\right)^{\perp} /\left(R_{\mathcal{E}_{1}} M\right)^{\perp}=I_{\mathcal{E}_{2}}\left(M^{*}\right) / I_{\mathcal{E}_{1}}\left(M^{*}\right) .
$$

Assuming $\operatorname{dim} M(G)$ is finite always and identifying $M$ with its double dual we have $\left(I_{\mathcal{E}}\left(M^{*}\right)\right)^{*}=R_{\mathcal{E}} M$ and $\left(R_{\mathcal{E}}\left(M^{*}\right)\right)^{*}=I_{\mathcal{E}} M$.

Proof. This is the same as [34, Prop. 2.2].

Proposition 4.1 says that the mappings $\mathcal{E} \mapsto I_{\mathcal{E}} M$ and $\mathcal{E} \mapsto R_{\mathcal{E}} M$ are order preserving and order reversing maps from the lattice of sets of isomorphism types of finite groups to the lattice of subfunctors of $M$. Using this we may construct filtrations of $M$ by ordering the isomorphism classes of finite groups as $1=H_{1}, H_{2}, H_{3}, \ldots$ in such a way that if $H_{i}$ is isomorphic to a section of $H_{j}$ then $i \leq j$. Let us write 
$M_{i}=I_{\left\{H_{1}, \ldots, H_{i}\right\}} M$ and $M^{i}=R_{\left\{H_{1}, \ldots, H_{i}\right\}} M$ with $M_{0}=0$ and $M^{0}=M$. We produce in this way an ascending filtration

$$
0=M_{0} \subseteq M_{1} \subseteq \cdots \subseteq M
$$

and a descending filtration

$$
M=M^{0} \supseteq M^{1} \supseteq \cdots
$$

of $M$ associated to the list $H_{1}, H_{2}, \ldots$ The last results apply to the terms of these filtrations, and we have for all $i$ :

(1) $(M \oplus N)_{i}=M_{i} \oplus N_{i}$;

(2) $M_{i} / M_{i-1}$ is generated by its value at $H_{i}$ and is only non-zero on groups which have $H_{i}$ as a section;

(3) $M_{i}=M^{* i *}$ when $R$ is a field and the values of $M$ are finite dimensional, with similar statements holding for the $M^{i}$.

We are interested in the factors $M_{i} / M_{i-1}$ and $M^{i-1} / M^{i}$ of these filtrations. Evidently these are associated in some way to the group $H_{i}$, but they depend also on the ordering of the rest of the list of isomorphism classes of groups and need not be uniquely determined by $H_{i}$. We will see from the next result that as the position of a particular group $H$ varies between different lists, there is a largest such factor associated to $H$, which occurs when $H$ appears as early as possible in a list. We will also be interested in the values of the factors $M_{i} / M_{i-1}$ and $M^{i-1} / M^{i}$ at $H_{i}$ which are in fact determined independently of the position of $H_{i}$ in the list of subgroups. To do this we make the following definitions.

The Brauer quotient of $M$ at $H$ is defined to be

$$
\bar{M}(H)=M(H) / \sum_{\substack{\gamma: J \rightarrow H \\ H \text { not a section of } J}} \operatorname{Im} M(\gamma) .
$$

We also define the restriction kernel of $M$ at $H$, namely

$$
\underline{M}(H)=\bigcap_{\substack{\gamma: H \rightarrow J \\ H \text { not a section of } J}} \operatorname{Ker} M(\gamma) .
$$

We have defined these constructions over an arbitrary commutative ring $R$ and when $R$ is a field our definitions agree in spirit with the usual ones. When $R$ is a local ring it is usual to factor out in addition the radical of the ring from these expressions (see [29]). This is something we do not do here, so that our terminology differs from that suggested by other contexts. One possibility would have been to devise a different name for our Brauer quotient, such as the 'transfer quotient' or the 'covariant quotient' but we have avoided doing this. 
The next result shows that a listing of finite groups with larger number of groups appearing prior to a given group $H$ gives rise to smaller quotients at the stage corresponding to $H$ in both the ascending and descending filtrations of $M$, while the values of these quotients at $H$ remain fixed.

Proposition 4.3. Let $M$ be a globally defined Mackey functor, let $H$ be a group and let $\mathcal{E}, \mathcal{F}$ be sets of finite groups with $\mathcal{E} \subseteq \mathcal{F}$.

(1) The inclusion mapping induces an epimorphism

$$
I_{\mathcal{E} \cup\{H\}} M / I_{\mathcal{E}} M \rightarrow I_{\mathcal{F} \cup\{H\}} M / I_{\mathcal{F}} M
$$

and a monomorphism

$$
R_{\mathcal{F}} M / R_{\mathcal{F} \cup\{H\}} M \rightarrow R_{\mathcal{E}} M / R_{\mathcal{E} \cup\{H\}} M .
$$

(2) If $\mathcal{E}$ is section-closed and contains every proper section of $H$, but not $H$ itself, then

$$
\left(I_{\mathcal{E} \cup\{H\}} M / I_{\mathcal{E}} M\right)(H) \cong \bar{M}(H)
$$

and

$$
\left(R_{\mathcal{E}} M / R_{\mathcal{E} \cup\{H\}} M\right)(H) \cong \underline{M}(H) .
$$

Proof. (1) The proof is the same as that of [34, Prop. 2.3(a)].

(2) We have from Proposition 4.1

$$
\begin{aligned}
I_{\mathcal{E}} M(H) & =\sum_{\substack{\gamma: G \rightarrow H \\
G \in \mathcal{E}}} \operatorname{Im} M(\gamma) \\
& =\sum_{\substack{\gamma: G \rightarrow H \\
G \text { a proper section of } H}} \operatorname{Im} M(\gamma)
\end{aligned}
$$

since every $\gamma: G \rightarrow H$ with $G \in \mathcal{E}$ factors through a proper section of $H$. Since $I_{\mathcal{E} \cup\{H\}} M(H)=M(H)$, the quotient is $\bar{M}(H)$. This establishes the first formula, and the proof of the second is very similar.

We conclude that the Brauer quotient and restriction kernel are dual in a certain sense.

Corollary 4.4. Let $M$ be a globally defined Mackey functor over a field $R$ whose values are all finite dimensional. For each group $H$ we have $\underline{M}(H)=\left(\overline{M^{*}}(H)\right)^{*}$.

Proof. This is a consequence of Proposition 4.2 and Proposition 4.3(2). 
Taking a list of isomorphism types of groups $1=H_{1}, H_{2}, \ldots$ in which the sections of each group $H_{i}$ always appear earlier than $H_{i}$, we see that for each group $G=H_{i}$ in the list there is a unique largest possibility for the functor $M_{i} / M_{i-1}$ obtained from a list in which $H_{i}$ appears as early as possible. This happens when $H_{1}, H_{2}, \ldots, H_{i}$ are exactly the sections of $H_{i}$. Let us write $\leq G$ for the set of sections of $G$ and $<G$ for the set of proper sections of $G$. We define

$$
M_{(G)}:=I_{\leq G} M / I_{<G} M \quad \text { and } \quad M^{(G)}:=R_{<G} M / R_{\leq G} M .
$$

We summarize the properties of these constructions.

Corollary 4.5. Let $1=H_{1}, H_{2}, \ldots$ be a list of groups in which the sections of each group $H_{i}$ always appear earlier than $H_{i}$ and let $M$ be a globally defined Mackey functor. In the ascending and descending filtrations of $M$, the factors $M_{i} / M_{i-1}$ and $M^{i-1} / M^{i}$ are only non-zero on groups which have $H_{i}$ as a section. We always have $\left(M_{i} / M_{i-1}\right)\left(H_{i}\right) \cong$ $\bar{M}\left(H_{i}\right)$ and $\left(M^{i-1} / M^{i}\right)\left(H_{i}\right) \cong M\left(H_{i}\right)$. In particular $M_{\left(H_{i}\right)}$ is only nonzero on groups which have $H_{i}$ as a section and $M_{\left(H_{i}\right)}\left(H_{i}\right)=\bar{M}\left(H_{i}\right)$. Furthermore $M_{i} / M_{i-1}$ is a homomorphic image of $M_{\left(H_{i}\right)}$ and $M^{i-1} / M^{i}$ is a subfunctor of $M^{\left(H_{i}\right)}$.

We conclude this section with a well-known result.

Proposition 4.6. Let $f: N \rightarrow M$ be a morphism of globally defined Mackey functors.

(1) If the composite morphism $N(H) \rightarrow M(H) \rightarrow \bar{M}(H)$ is surjective for every subgroup $H$ then $f$ is an epimorphism.

(2) If the composite morphism $\underline{N}(H) \rightarrow N(H) \rightarrow M(H)$ is injective for every subgroup $H$ then $f$ is an monomorphism.

Proof. The proof is the same as the proof of [34, Prop. 2.5].

(1) Let $0=M_{0} \subseteq M_{1} \subseteq \cdots$ be an ascending filtration of $M$ in the sense defined after Proposition 4.2. We show by induction that $M_{i} \subseteq \operatorname{Im} f$, which is certainly true when $i=0$. Assuming that $M_{i-1} \subseteq \operatorname{Im} f$ we also know that $\left(M_{i} \cap \operatorname{Im} f\right) / M_{i-1}=M_{i} / M_{i-1}$ since this is generated by $\left(M_{i} / M_{i-1}\right)\left(H_{i}\right)$ by Proposition $4.1(4)$, which equals $\bar{M}\left(H_{i}\right)$ by Proposition $4.3(2)$ and is covered by $f$. Hence $M_{i} \subseteq \operatorname{Im} f$. Since $M=\bigcup M_{i}$ we have $M \subseteq \operatorname{Im} f$.

The proof of (2) is dual.

\section{The Functors $\Delta_{H, V}$ AND $\nabla_{H, V}$}

We now construct the functors which play the role of standard and costandard objects in $\operatorname{Mack}_{R}^{\mathcal{X}, \mathcal{Y}}$. The idea to consider functors of this 
type can be traced back at least to Lewis [24]. The functors which we call $\nabla_{H, V}$ here were also defined in a more restricted setting in [32] where they were denoted $J_{H, V}$. We choose to define these functors by a direct explicit construction and in subsequent sections we will need to know some of the information which this produces. The functors can also be defined using the adjoint properties which they satisfy and I am grateful to the referee for pointing out that this approach can also be made explicit and may be shorter. We sketch the approach after Proposition 5.2. The functors we will define will have as their domain a section-closed set of finite groups $\mathcal{D}$. However, none of the definitions or arguments depend on $\mathcal{D}$ and so we omit $\mathcal{D}$ from the notation.

We fix a finite group $H$, and for each finite group $G$ we consider the set of sections of $G$ isomorphic to $H$ with kernel in $\mathcal{X}$. To be very specific about this we define

$$
\operatorname{Sec}_{\mathcal{X}}(G, H)=\{\alpha: A \rightarrow H \mid A \leq G, \operatorname{Ker} \alpha \in \mathcal{X}\} .
$$

Whenever $V$ is an $R$ Out $H$-module we put

$$
\Delta_{H, V}^{\mathcal{X}}(G)=\left(\bigoplus_{\alpha \in \operatorname{Sec}_{\mathcal{X}}(G, H)}{ }^{\alpha} V\right)_{G \times \operatorname{Aut} H}
$$

and

$$
\nabla_{H, V}^{\mathcal{Y}}(G)=\left(\bigoplus_{\alpha \in \operatorname{Sec} \mathcal{Y}(G, H)}{ }^{\alpha} V\right)^{G \times \operatorname{Aut} H}
$$

where each ${ }^{\alpha} V$ is a copy of $V$, the subscript and superscript $G \times$ Aut $H$ mean fixed quotient and fixed points, and there is an action of $G \times$ Aut $H$ on the direct sum (before taking fixed quotient and fixed points) specified as follows: if $v={ }^{\alpha} v \in{ }^{\alpha} V, g \in G$ and $\gamma \in$ Aut $H$ then

$$
(g, \gamma)\left({ }^{\alpha} v\right)={ }^{\gamma \alpha c_{g^{-1}}} \gamma(v)
$$

where the (left) superscript on a vector indicates the copy of $V$ to which it belongs. Here $c_{g}(x)=g x g^{-1}$ is conjugation by $g$.

We describe more fully the structure as $G \times$ Aut $H$-modules of the direct sums which appear in the definitions of $\Delta_{H, V}$ and $\nabla_{H, V}$. There is an action of $G \times$ Aut $H$ on each of $\operatorname{Sec}_{\mathcal{X}}(G, H)$ and $\operatorname{Sec}_{\mathcal{Y}}(G, H)$ given by composition, as follows: if $\phi \in$ Aut $H$ and $g \in G$, for any $\alpha: A \rightarrow H$, we have

$$
(g, \phi) \cdot \alpha: A^{g \stackrel{c_{g}-1}{\longrightarrow}} A \stackrel{\alpha}{\longrightarrow} H \stackrel{\phi}{\longrightarrow} H .
$$

We see that in the action of $G \times$ Aut $H$ on the direct sum given above, the subspaces ${ }^{\alpha} V$ are permuted in the way just given, and the direct sum is an induced module.

For any section $\alpha: A \rightarrow H$ where $A \leq G$ we define

$$
N_{G}(\alpha)=N_{G}(A) \cap N_{G}(\operatorname{Ker}(\alpha)) .
$$


There is a homomorphism $N_{G}(\alpha) \rightarrow \operatorname{Aut}(H)$ which sends $g \in N_{G}(\alpha)$ to the automorphism of $H$ induced by $c_{g}$. The stabilizer in $G \times \operatorname{Aut} H$ of the subspace ${ }^{\alpha} V$ is

$$
\begin{aligned}
\operatorname{Stab}_{G \times \operatorname{Aut} H}\left({ }^{\alpha} V\right) & =\left\{(g, \gamma) \mid \gamma \alpha c_{g^{-1}}=\alpha\right\} \\
& =\left\{(g, \gamma) \mid c_{g} \in N_{G}(\alpha), \gamma \text { is induced by } c_{g}\right\} \\
& \cong N_{G}(\alpha)
\end{aligned}
$$

via the diagonal embedding $N_{G}(\alpha) \rightarrow G \times$ Aut $H$ which has components the inclusion $N_{G}(\alpha) \hookrightarrow G$ and the homomorphism $N_{G}(\alpha) \rightarrow$ Aut $H$. In the given action of $G \times$ Aut $H$ on $\bigoplus^{\alpha} V$, this diagonal subgroup acts on ${ }^{\alpha} V$ via projection to Aut $H$, so identifying it with $N_{G}(\alpha)$ the action on ${ }^{\alpha} V$ is via the map $N_{G}(\alpha) \rightarrow$ Aut $H$. Now

$$
\bigoplus_{\alpha \in \operatorname{Sec} \mathcal{X}^{\prime}(G, H)}{ }^{\alpha} V \cong \bigoplus_{\alpha \in\left[\operatorname{Aut} H \backslash \operatorname{Sec} \mathcal{X}_{(G, H) / G]}\right.}{ }^{\alpha} V \uparrow_{N_{G}(\alpha)}^{G \times \operatorname{Aut} H}
$$

Thus we have the following expressions for $\Delta$ and $\nabla$.

\section{Proposition 5.1.}

$$
\Delta_{H, V}(G) \cong \bigoplus_{\alpha \in\left[\text { Aut } H \backslash \operatorname{Sec}_{\mathcal{X}}(G, H) / G\right]}{ }^{\alpha} V_{N_{G}(\alpha)}
$$

and

$$
\nabla_{H, V}(G) \cong \bigoplus_{\alpha \in\left[\text { Aut } H \backslash \operatorname{Sec} \mathcal{Y}_{(G, H) / G]}\right.}{ }^{\alpha} V^{N_{G}(\alpha)}
$$

Proof. We take fixed quotients and fixed points in the previous expression and use the isomorphisms $\left(W \uparrow_{L}^{G}\right)_{G} \cong W_{L}$ and $\left(W \uparrow_{L}^{G}\right)^{G} \cong W^{L}$ for the fixed quotients and fixed points of an induced module.

We now make $\Delta_{H, V}$ into a functor, and define first a covariant morphism

$$
\phi_{*}: \Delta_{H, V}(G) \rightarrow \Delta_{H, V}\left(G_{1}\right)
$$

associated to any group homomorphism $\phi: G \rightarrow G_{1}$. (We only need to define $\phi_{*}$ when $\operatorname{Ker} \phi \in \mathcal{Y}$, but this condition does not make an appearance in the definition.) Suppose that $\alpha: A \rightarrow H$ is a section of $G$, where $A \leq G$, and consider $\left.\phi\right|_{A}: A \rightarrow G_{1}$. It may happen that $\operatorname{Ker}\left(\left.\phi\right|_{A}\right) \subseteq \operatorname{Ker} \alpha$, and in this case we can factor $\alpha$ as $\alpha=\left.\hat{\alpha} \circ \phi\right|_{A}$ where $\hat{\alpha}: \phi(A) \rightarrow H$ is a section of $G_{1}$. We may express this situation by saying that there is a commutative diagram

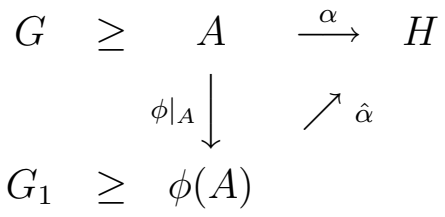


Observe in this case that $\operatorname{Ker} \hat{\alpha} \cong \operatorname{Ker} \alpha / \operatorname{Ker}\left(\left.\phi\right|_{A}\right) \in \mathcal{X}$ since $\mathcal{X}$ is section-closed. With this notation we define a morphism

$$
\bar{\phi}_{*}: \bigoplus_{\alpha \in \operatorname{Sec} \mathcal{X}(G, H)}{ }^{\alpha} V \rightarrow \bigoplus_{\alpha \in \operatorname{Sec} \mathcal{X}\left(G_{1}, H\right)}{ }^{\alpha} V
$$

by

$$
\bar{\phi}_{*}\left({ }^{\alpha} v\right)= \begin{cases}{ }^{\alpha} v & \text { if }\left.\operatorname{Ker} \phi\right|_{A} \subseteq \operatorname{Ker} \alpha \\ 0 & \text { otherwise. }\end{cases}
$$

To define $\phi_{*}$ we observe that $\bar{\phi}_{*}$ commutes with the action of $G \times$ $\operatorname{Aut}(H)$ on the direct sums, and so induces a map on fixed quotients. We define $\phi_{*}$ to be the composite $\phi_{*}=$ cores $\circ \bar{\phi}_{*}$, where cores is the canonical projection of fixed quotients between $\phi G \times$ Aut $H$ and $G_{1} \times$ Aut $H$, as follows:

$$
\begin{aligned}
&\left(\bigoplus_{\alpha \in \operatorname{Sec} \mathcal{X}(G, H)}{ }^{\alpha} V\right)_{G \times \operatorname{Aut} H} \stackrel{\bar{\phi}_{*}}{\longrightarrow}\left(\bigoplus_{\alpha \in \operatorname{Sec} \mathcal{X}^{\prime}\left(G_{1}, H\right)}{ }^{\alpha} V\right)_{\phi G \times \operatorname{Aut} H} \\
& \stackrel{\text { cores }}{\longrightarrow}\left(\bigoplus_{\alpha \in \operatorname{Sec} \mathcal{X}^{\prime}\left(G_{1}, H\right)}{ }^{\alpha} V\right)_{G_{1} \times \operatorname{Aut} H} .
\end{aligned}
$$

Next we define the contravariant morphism $\phi^{*}: \Delta_{H, V}(G) \rightarrow \Delta_{H, V}\left(G_{1}\right)$ associated to a group homomorphism $\phi: G_{1} \rightarrow G$ for which $\operatorname{Ker} \phi \in \mathcal{X}$. If $\alpha: A \rightarrow H$ is a section of $G$, where $A \leq G$, we consider the subgroup $\phi^{-1} A \leq G_{1}$, and it may happen that the composite $\tilde{\alpha}=\left.\alpha \phi\right|_{\phi^{-1} A}$ : $\phi^{-1} A \rightarrow H$ is surjective. In this situation Ker $\tilde{\alpha} \in \mathcal{X}$ since it appears in a short exact sequence $1 \rightarrow \operatorname{Ker} \phi \rightarrow \operatorname{Ker} \tilde{\alpha} \rightarrow \operatorname{Ker} \alpha \cap \phi \phi^{-1} A \rightarrow 1$ and $\mathcal{X}$ is extension-closed. We define

$$
\bar{\phi}^{*}: \bigoplus_{\alpha \in \operatorname{Sec} \mathcal{X}(G, H)}{ }^{\alpha} V \rightarrow \bigoplus_{\alpha \in \operatorname{Sec} \mathcal{X}\left(G_{1}, H\right)}{ }^{\alpha} V
$$

by

$$
\bar{\phi}^{*}\left({ }^{\alpha} v\right)= \begin{cases}\tilde{\alpha}_{v} & \text { if } \tilde{\alpha} \text { is surjective } \\ 0 & \text { otherwise. }\end{cases}
$$

This time the non-zero situation may be expressed by saying there is a commutative diagram

$$
\begin{aligned}
& G_{1} \geq \phi^{-1} A \stackrel{\tilde{\alpha}}{\longrightarrow} H \\
& \phi \downarrow \quad \nearrow \alpha \\
& G \geq A
\end{aligned}
$$

Again $\bar{\phi}^{*}$ commutes with the action of $G_{1} \times$ Aut $H$ on the direct sums and induces a map on fixed quotients. We define $\phi^{*}$ to be the composite 
$\phi^{*}=\bar{\phi}^{*}$ o res, where res is the restriction map in group homology (the relative trace) between $G$ and $\phi G_{1}$, as follows:

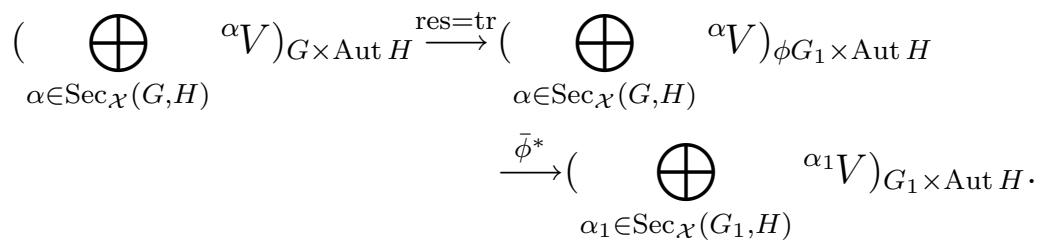

We may show that $\Delta_{H, V}$ is indeed a globally defined Mackey functor with respect to $\mathcal{X}$ and $\mathcal{Y}$. This may be done by verifying that axioms $(1)-(5)$ of Section 2 are satisfied, which is a routine but notationally complex task.

We similarly make $\nabla_{H, V}$ into a functor by using the operations dual to those of $\Delta_{H, V}$. Suppose that $\phi: G \rightarrow G_{1}$ is a group homomorphism with $\operatorname{Ker} \phi \in \mathcal{Y}$. We define $\phi_{*}: \nabla_{H, V}(G) \rightarrow \nabla_{H, V}\left(G_{1}\right)$. As before, if $\alpha: A \rightarrow H$ is a section of $G$, where $A \leq G$ and $\operatorname{Ker} \alpha \in \mathcal{X}$, we put $\alpha=\hat{\alpha} \phi$ if $\operatorname{Ker}\left(\left.\phi\right|_{A}\right) \subseteq \operatorname{Ker} \alpha$. We may express this by saying that there is a commutative diagram

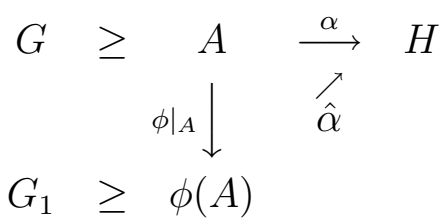

Note in this case that $\operatorname{Ker} \hat{\alpha} \cong \operatorname{Ker} \alpha /\left(\left.\operatorname{Ker} \phi\right|_{A}\right) \in \mathcal{Y}$ since $\mathcal{Y}$ is sectionclosed. In the direct sum $\bigoplus_{\alpha \in \operatorname{Sec} \mathcal{Y}(G, H)}{ }^{\alpha} V$ we put

$$
\bar{\phi}_{*}\left({ }^{\alpha} v\right)= \begin{cases}\hat{\alpha}_{v} & \text { if } \operatorname{Ker}\left(\left.\phi\right|_{A}\right) \subseteq \operatorname{Ker} \alpha \\ 0 & \text { otherwise. }\end{cases}
$$

Now $\bar{\phi}_{*}$ commutes with the action of $G \times$ Aut $\underline{H}$ on the direct sum, and we define $\phi_{*}$ to be the composite $\phi_{*}=$ cores $\circ \bar{\phi}_{*}$ as follows:

$$
\begin{aligned}
\left(\bigoplus_{\alpha \in \operatorname{Sec} \mathcal{Y}(G, H)}{ }^{\alpha} V\right)^{G \times \operatorname{Aut} H} \stackrel{\bar{\phi}_{*}}{\longrightarrow} & \left(\bigoplus_{\substack{\alpha_{1} \in \operatorname{Sec} \mathcal{Y}\left(G_{1}, H\right) \\
\text { cores }}}^{\longrightarrow} \bigoplus_{\alpha_{1} \in \operatorname{Sec} \mathcal{Y}\left(G_{1}, H\right)}^{\alpha_{1} V}\right)^{\phi G \times \text { Aut } H}
\end{aligned}
$$

If $\phi: G_{1} \rightarrow G$ and $\operatorname{Ker} \phi \in \mathcal{X}$ we put

$$
\bar{\phi}^{*}\left({ }^{\alpha} v\right)=\sum_{G_{1} \geq K_{1} \stackrel{\phi}{\rightarrow} A \stackrel{\alpha}{\rightarrow} H} \alpha \phi_{v}
$$

where the sum is over all subgroups $K_{1} \leq G_{1}$ such that $\alpha \phi\left(K_{1}\right)=H$ and $K_{1} \cap \operatorname{Ker} \alpha \phi \in \mathcal{Y}$. Now $\bar{\phi}^{*}$ commutes with the action of $G_{1} \times$ Aut $H$ 
and we define $\phi^{*}=\bar{\phi}^{*}$ ores as follows:

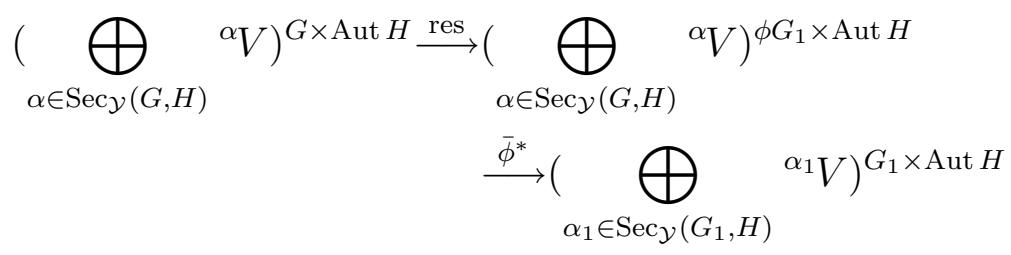

where res is the restriction map in group cohomology from $G \times$ Aut $H$ to $\phi G_{1} \times$ Aut $H$. In the same way as before we may verify that $\nabla_{H, V}$ is a globally defined Mackey functor using the axioms in Section 2 .

The functors $\nabla_{H, V}$ enjoy the same formal properties as the functors $J_{H, V}$ defined in [24] and [32], and the $\Delta_{H, V}$ enjoy dual properties. Perhaps the most important of these is an adjoint property.

Proposition 5.2. For each subgroup $H$ the functor

$$
\operatorname{Mack}_{R}^{\mathcal{X}, \mathcal{Y}} \rightarrow R \text { Out } H \text {-mod }
$$

specified by $M \mapsto \underline{M}(H)$ has left adjoint $V \mapsto \Delta_{H, V}$. Similarly the functor specified by $M \mapsto \bar{M}(H)$ has right adjoint $V \mapsto \nabla_{H, V}$. The natural isomorphisms

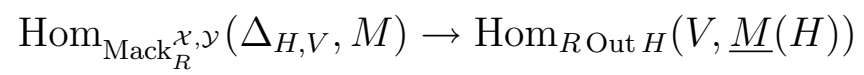

and

$$
\operatorname{Hom}_{\operatorname{Mack}_{R}^{\mathcal{X}, \mathcal{Y}}}\left(M, \nabla_{H, V}\right) \rightarrow \operatorname{Hom}_{R} \text { Out } H(\bar{M}(H), V)
$$

are given by evaluating morphisms at $H$.

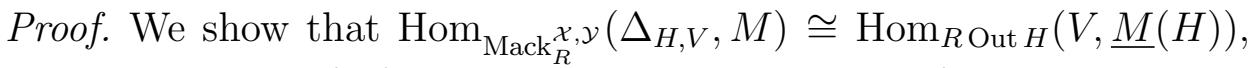
omitting the proof of the corresponding statement for $\nabla_{H, V}$, since it is similar. The isomorphism from left to right is $\eta \mapsto \eta_{H}$, namely the effect of the natural transformation $\eta$ at the group $H$. Observe that the image of the $R \operatorname{Out}(H)$-module homomorphism $\eta_{H}: V \rightarrow M(H)$ is indeed contained in $\underline{M}(H)$ since if $\gamma: H \rightarrow J$ is a morphism where $H$ is not a section of $J$ then $\Delta_{H, V}(J)=0$ and since the diagram

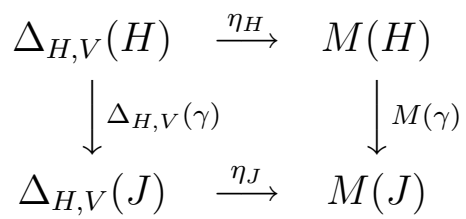

commutes we deduce that $M(\gamma) \eta_{H}=0$.

For the isomorphism in the direction right to left, suppose that $\theta: V \rightarrow M(H)$ is an $R$ Out $H$-module homomorphism. We define a natural transformation $\underline{\theta}_{G}: \Delta_{H, V}(G) \rightarrow M(G)$ as follows. First we define a map $\theta^{\prime}: \bigoplus_{\alpha \in \operatorname{Sec} \mathcal{X}(G, H)}{ }^{\alpha} V \rightarrow M(G)$ by specifying that it has 
component $\iota_{*} \alpha^{*} \theta$ on the summand ${ }^{\alpha} V$, where $H$ is expressed as a section of $G$ by maps $G \stackrel{\iota}{\longleftarrow} K \stackrel{\alpha}{\rightarrow} H$. By computation we see that the action of $G \times$ Aut $H$ on the direct sum is trivialized after applying $\theta^{\prime}:$ if $g \in G$, $\gamma \in$ Aut $G$ and ${ }^{\alpha} v \in{ }^{\alpha} V$ we have

$$
\begin{aligned}
\theta^{\prime}\left((g, \gamma)\left({ }^{\alpha} v\right)\right) & =\theta^{\prime}\left({ }^{\gamma \alpha c_{g}-1} \gamma(v)\right) \\
& =\iota_{*}\left(\gamma \alpha c_{g^{-1}}\right)^{*} \theta(\gamma(v)) \\
& =\iota_{*} c_{g} \alpha^{*} \gamma^{*} \gamma_{*} \theta(v) \\
& =\iota_{*} \alpha^{*} \theta(v) \\
& =\theta^{\prime}\left({ }^{\alpha} v\right) .
\end{aligned}
$$

Thus $\theta^{\prime}$ induces a map $\underline{\theta}: \Delta_{H, V}(G) \rightarrow M(G)$ on the fixed quotient.

The proof concludes with a verification that $\underline{\theta}$ is indeed a natural transformation, and that the constructions $\eta \mapsto \eta_{H}$ and $\theta \mapsto \phi$ are mutually inverse, which may be checked by computation.

The adjoint properties of Proposition 5.2 may be used to define $\Delta_{H, V}$ and $\nabla_{H, V}$, and this approach to the definition is much faster than the one we have taken. It does, however, leave the question of whether such adjoints exist, and does not immediately give an explicit description of the values of the functors. I am grateful to the referee for pointing out that an explicit description may be deduced as follows. We define

$$
\nabla_{H, V}(K):=\operatorname{Hom}_{R \text { Out } H}\left(\overline{A_{R}^{\mathcal{X}, \mathcal{Y}}(, K)}(H), V\right)
$$

noting with hindsight that the term on the right is isomorphic to

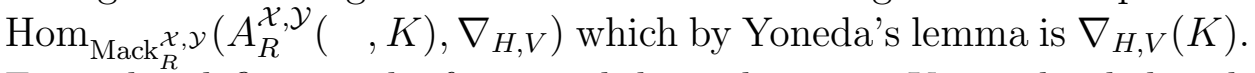
From this definition the functorial dependence on $K$ may be deduced, as well as the fact that the definition extends to the natural isomorphism of Proposition 5.2. An expression for

$$
\overline{A_{R}^{\mathcal{X}, \mathcal{Y}}(, K)}(H)=A_{R}^{\mathcal{X}, \mathcal{Y}}(, K)_{(H)}(H)
$$

is given in step 2 of the proof of Theorem 6.3, and this immediately yields the description in Proposition 5.1. When $V$ is finite dimensional we can deduce the corresponding properties for $\Delta_{H, V}$ using the duality statement of Corollary 5.9.

Corollary 5.3. Let $H$ be a group and let $\mathcal{H}$ be the full subcategory of $\operatorname{Mack}_{R}^{\mathcal{X}, \mathcal{Y}}$ whose objects are the functors $M$ with $M(K)=0$ if $K$ is a proper section of $H$. The functor $M \mapsto M(H)$ from $\mathcal{H}$ to $R$ Out $H$-mod has left adjoint $V \mapsto \Delta_{H, V}$ and right adjoint $V \mapsto \nabla_{H, V}$. Thus there 
are natural isomorphisms

$$
\begin{aligned}
& \operatorname{Hom}_{\mathcal{H}}\left(\Delta_{H, V}, M\right) \cong \operatorname{Hom}_{R \text { Out } H}(V, M(H)) \\
& \operatorname{Hom}_{\mathcal{H}}\left(M, \nabla_{H, V}\right) \cong \operatorname{Hom}_{R \text { Out } H}(M(H), V)
\end{aligned}
$$

Proof. For such a globally defined Mackey functor $M$ we have $\bar{M}(H)=$ $\underline{M}(H)=M(H)$ and so the adjunction isomorphisms of Proposition 5.2 become the ones stated.

Several properties of the $\Delta$ and $\nabla$ functors follow from their identification as adjoints, and we state these in the next results.

Corollary 5.4. Let $\mathcal{E} \subseteq \mathcal{D}$ be sets of finite groups which are closed under taking sections and let $H$ be a group in $\mathcal{E}$. Writing ${ }^{\mathcal{E}} \Delta_{H, V}$ and ${ }^{\mathcal{D}} \Delta_{H, V}$ for the functors defined on $\mathcal{E}$ and $\mathcal{D}$ we have ${ }^{\mathcal{E}} \Delta_{H, V}={ }^{\mathcal{D}} \Delta_{H, V} \downarrow_{\mathcal{E}}^{\mathcal{D}}$ and ${ }^{\mathcal{D}} \Delta_{H, V} \cong{ }^{\mathcal{E}} \Delta_{H, V} \uparrow_{\mathcal{E}}^{\mathcal{D}}$. Furthermore ${ }^{\mathcal{D}} \Delta_{H, V}$ is generated by its value at $H$, and has the set of sections of $H$ as its vertex.

Proof. The definition of ${ }^{\mathcal{E}} \Delta_{H, V}$ is the same as the definition of ${ }^{\mathcal{D}} \Delta_{H, V}$ on groups in $\mathcal{E}$, and this establishes the restriction formula. For the induction formula we exploit the fact that $V \mapsto{ }^{\mathcal{D}} \Delta_{H, V}$ is left adjoint to $M \mapsto \underline{M}(H)$. The latter functor can be written as a composite $M \mapsto M \underline{\downarrow_{\mathcal{E}}^{\mathcal{D}}} \longmapsto \underline{M}(H)$ and so its left adjoint factors as a composite of left adjoints $V \mapsto{ }^{\mathcal{E}} \Delta_{H, V} \mapsto{ }^{\mathcal{E}} \Delta_{H, V} \uparrow_{\mathcal{E}}^{\mathcal{D}}$. The isomorphism follows from this.

We see that ${ }^{\mathcal{D}} \Delta_{H, V}$ is projective relative to every set of finite groups which contains the sections of $H$, and it is not projective to any smaller set since it is zero on proper sections of $H$. Thus the vertex is as claimed.

Any functor is generated by its values on groups in its vertex, and since $H$ is the only such group on which ${ }^{\mathcal{D}} \Delta_{H, V}$ is non-zero, this functor is generated by its value at $H$.

Evidently a similar statement to that of Corollary 5.4 is true for the $\nabla_{H, V}$ provided we develop a theory of relative injectivity dual to that of relative projectivity, using the right adjoint to the restriction functor instead of the left. In any case it is clear that $\nabla_{H, V}$ is cogenerated by its value at $H$.

Corollary 5.5. Let $H$ be a group and let $V$ be an $R$ Out $H$-module which has a unique simple quotient $U$ (up to isomorphism). Then $\Delta_{H, V}$ has a unique simple quotient $S_{H, U}$. Dually, if $V$ has a simple socle $U$ then $\nabla_{H, V}$ has a simple socle isomorphic to $S_{H, U}$. If $R$ is a field or a complete discrete valuation ring then $\Delta_{H, V}$ is an image of the indecomposable projective $P_{H, U}$ and $\nabla_{H, V}$ is isomorphic to a subfunctor of the indecomposable injective $I_{H, U}$. 
Proof. The natural transformation $\Delta_{H, V} \rightarrow S_{H, U}$, which by Corollary 5.3 extends the homomorphism $V \rightarrow U$, is non-zero, so it is an epimorphism since $S_{H, U}$ is simple. If a simple functor $S_{K, W}$ is an image of $\Delta_{H, V}$ then $H$ is a section of $K$ (since $\Delta_{H, V}(K)=0$ unless $H$ is a section of $K$ ), and in fact we must have $K \cong H$ since otherwise $S_{K, W}(H)=0$ and the map from $\Delta_{H, V}$ would be zero by Corollary 5.3. We now see that $U \cong W$ since $S_{H, W}(H)=W$ is a simple image of $V$. The statement about $\nabla_{H, V}$ is proved dually, and the remaining statements are immediate consequences of what we have already shown.

The following interpretation is well known and will be used in Section 11 when we compute the values of simple functors.

Corollary 5.6. Let $V$ be a simple $R \operatorname{Out}(H)$-module. Then $S_{H, V} \cong$ $\Delta_{H, V} / R_{\{H\}} \Delta_{H, V}$ is the quotient of $\Delta_{H, V}$ cogenerated by its value at $H$, and $S_{H, V} \cong I_{\{H\}} \nabla_{H, V}$ is the subfunctor of $\nabla_{H, V}$ generated by its value at $H$.

Proof. By Corollary 5.5 the simple socle of $\nabla_{H, V}$ contains $\nabla_{H, V}(H)$, and hence is generated by this value. The statement about $\Delta_{H, V}$ is dual.

Corollary 5.7. Consider the ascending and descending filtrations of a Mackey functor $M$ associated to a list $1=H_{1}, H_{2}, \ldots$ of isomorphism types of groups, listed so that the sections of each $H_{i}$ always appear earlier than $H_{i}$. For each $i$ there is an epimorphism

$$
\Delta_{H_{i}, \bar{M}\left(H_{i}\right)} \rightarrow M_{i} / M_{i-1}
$$

and a monomorphism

$$
M^{i-1} / M^{i} \rightarrow \nabla_{H_{i}, \underline{M}}\left(H_{i}\right)
$$

Proof. By Corollary 4.5 we know that $M_{i} / M_{i-1}$ and $M^{i-1} / M^{i}$ vanish on groups which do not have $H_{i}$ as a section, and we have $\left(M_{i} / M_{i-1}\right)\left(H_{i}\right)=$ $\bar{M}\left(H_{i}\right)$ and $\left(M^{i-1} / M^{i}\right)\left(H_{i}\right)=\underline{M}\left(H_{i}\right)$. Thus by the adjoint property of 5.3 we have morphisms as claimed. Since $M_{i} / M_{i-1}$ is generated by its value at $H_{i}$ the first is an epimorphism, and since $M^{i-1} / M^{i}$ is cogenerated by its value at $H_{i}$ the second is a monomorphism.

The last result allows us to bound the dimension of the evaluation of a globally defined Mackey functor in terms of its Brauer quotients and restriction kernels. 
Corollary 5.8. Let $M$ be a globally defined Mackey functor defined over a field $R$. For each group $G$ we have

$$
\operatorname{dim} M(G) \leq \sum_{\substack{H \text { a section of } G \\ \text { up to isomorphism }}} \operatorname{dim} \Delta_{H, \bar{M}(H)}(G)
$$

and

$$
\operatorname{dim} M(G) \leq \sum_{\substack{H \text { a section of } G \\ \text { up to isomorphism }}} \operatorname{dim} \nabla_{H, \underline{M(H)}}(G) .
$$

Proof. We take a list $1=H_{1}, H_{2}, \ldots$ of isomorphism types of groups, so that the sections of each $H_{i}$ always appear earlier than $H_{i}$. Now $\operatorname{dim} M(G)=\sum \operatorname{dim}\left(M_{i} / M_{i-1}\right)(G)$ and each of these dimensions in the sum is bounded by $\operatorname{dim} \Delta_{H_{i}, \bar{M}\left(H_{i}\right)}(G)$, by Corollary 5.7. Note that $M_{i} / M_{i-1}(G)$ and $\Delta_{H_{i}, \bar{M}\left(H_{i}\right)}(G)$ are only non-zero when $H_{i}$ is a section of $G$, so that the sums we are dealing with are finite. The second inequality is proved similarly.

The bounds given in Corollary 5.8 are best possible in general. Indeed, it will be proved that the first inequality is an equality whenever $M$ is projective, from which we can deduce by duality that the second inequality is an equality whenever $M$ is injective.

We next state the behaviour of $\Delta_{H, V}$ and $\nabla_{H, V}$ under the duality between $\operatorname{Mack}_{R}^{\mathcal{X}, \mathcal{Y}}$ and $\operatorname{Mack}_{R}^{\mathcal{Y}, \mathcal{X}}$ and for this we write the functors in $\operatorname{Mack}_{R}^{\mathcal{X}, \mathcal{Y}}$ as $\Delta_{H, V}^{\mathcal{X}}$ and $\nabla_{H, V}^{\mathcal{Y}}$.

Corollary 5.9. Assume that $R$ is a field, let $H$ be a group and let $V$ be a finite dimensional $R$ Out $H$-module. We have $\left(\Delta_{H, V}^{\mathcal{X}}\right)^{*} \cong \nabla_{H, V^{*}}^{\mathcal{X}}$ and $\left(\nabla_{H, V}^{\mathcal{Y}}\right)^{*} \cong \Delta_{H, V^{*}}^{\mathcal{Y}}$ as functors in $\operatorname{Mack}_{R}^{\mathcal{Y}, \mathcal{X}}$.

Proof. We observe that $\left(\Delta_{H, V}^{\mathcal{X}}\right)^{*}(H)=V^{*}$, and also that $\left(\Delta_{H, V}^{\mathcal{X}}\right)^{*}$ satisfies the right adjoint property which characterizes $\nabla_{H, V^{*}}^{\mathcal{X}}$, thus establishing the first isomorphism. The second follows similarly.

We may use the adjoint properties of the $\Delta$ and $\nabla$ functors to give information about the Ext groups of simple globally defined Mackey functors. The approach we take is exactly the same as in [31, Section $14]$ and the arguments presented there go through here also.

Theorem 5.10. Let $S_{H, V}, S_{K, W}$ be simple globally defined Mackey functors over a field $R$.

(1) If $\operatorname{Ext}_{\mu_{R}^{x, y}}^{1}\left(S_{H, V}, S_{K, W}\right) \neq 0$ then either $H$ is a section of $K$ or $K$ is a section of $H$. 
(2) If $H=K$ then

$$
\begin{aligned}
\operatorname{dim} \operatorname{Ext}\left(S_{H, V}, S_{H, W}\right)= & \text { multiplicity of } S_{H, W} \\
& \text { in the second Loewy layer of } \Delta_{H, P_{V}} . \\
= & \text { multiplicity of } S_{H, V} \\
& \text { in the second socle layer of } \nabla_{H, P_{W}}
\end{aligned}
$$

The evaluation at $H$ induces a morphism

$$
\operatorname{Ext}_{\mu_{R}^{\mathcal{X}, \mathcal{Y}}}^{1}\left(S_{H, V}, S_{H, W}\right) \rightarrow \operatorname{Ext}_{R \text { Out } H}^{1}(V, W)
$$

which is injective. In particular

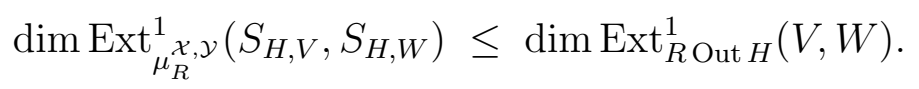

(3) If $K$ is a proper section of $H$ then

$$
\begin{aligned}
\operatorname{dim} \operatorname{Ext}_{\mu_{R}^{\mathcal{X}, \mathcal{Y}}}\left(S_{H, V}, S_{K, W}\right)= & \text { multiplicity of } S_{H, V} \\
& \text { in the second socle layer of } \nabla_{K, W} .
\end{aligned}
$$

(4) If $H$ is a proper section of $K$ then

$$
\begin{aligned}
\operatorname{dim} \operatorname{Ext}_{\mu_{R}^{\mathcal{X}, \mathcal{Y}}}\left(S_{H, V}, S_{K, W}\right)= & \text { multiplicity of } S_{K, W} \\
& \text { in the second Loewy layer of } \Delta_{H, V} .
\end{aligned}
$$

We comment that by virtue of the duality between $\operatorname{Mack}_{R}^{\mathcal{X}, \mathcal{Y}}$ and $\operatorname{Mack}_{R}^{\mathcal{Y}, \mathcal{X}}$ each of statements (3) and (4) is equivalent to another statement in the dual category in which multiplicities in the second Loewy layer of a $\Delta$ are interchanged with multiplicities in the second socle layer of a $\nabla$. We leave it to the reader to formulate this.

Proof. Suppose we have a non-split extension $0 \rightarrow S_{K, W} \rightarrow M \rightarrow$ $S_{H, V} \rightarrow 0$ and that $K$ is not a section of $H$. We will show that $H$ is a section of $K$. This Mackey functor $M$ vanishes on groups which are proper sections of $H$ and so by Corollary 5.3 the identity map on $M(H)$ extends to a morphism $\Delta_{H, M(H)} \rightarrow M$. Since the extension is non-split, $M$ has $S_{H, V}$ as its unique simple quotient, and so is generated by its value at $H$. It follows that $M$ is an image of $\Delta_{H, M(H)}$, and this is a functor which is only non-zero on groups which have $H$ as a section. Thus $K$ has $H$ as a section. This proves part (1).

In the proof of the remaining parts we use the fact that

$$
\operatorname{dim} \operatorname{Ext}_{\mu_{R}^{\mathcal{X}, \mathcal{Y}}}\left(S_{H, V}, S_{K, W}\right)=n
$$

if and only if $n$ is the largest number $r$ for which there is an extension

$$
0 \rightarrow\left(S_{K, W}\right)^{r} \rightarrow M \rightarrow S_{H, V} \rightarrow 0
$$

in which $S_{H, V}$ is the semisimple quotient of $M$. 
Assume that $H=K$. To prove (2) we observe by Corollary 5.5 that $\Delta_{H, P_{V}}$ has a simple top isomorphic to $S_{H, V}$, and so if $S_{H, W}$ occurs with multiplicity $r$ in the second Loewy layer of $\Delta_{H, P_{V}}$ then an extension $\left(^{*}\right)$ exists. On the other hand, given an extension $\left(^{*}\right)$ we may lift the projective cover $P_{V} \rightarrow V$ to an $R \operatorname{Out}(H)$-module hommorphism $P_{V} \rightarrow$ $M(H)$ which corresponds by Corollary 5.3 to a morphism $\Delta_{H, P_{V}} \rightarrow M$ which is an epimorphism since it surjects onto $S_{H, V}$, the composite $P_{V} \rightarrow M(H) \rightarrow V$ being surjective. This shows that $S_{H, V}$ occurs at least $r$ times in the second Loewy layer of $\Delta_{H, P_{V}}$ thus proving the first equality of (2). The second equality is proved by a dual argument.

We prove that the morphism $\operatorname{Ext}_{\mu_{R}^{\mathcal{X}, \mathcal{Y}}}\left(S_{H, V}, S_{H, W}\right) \rightarrow \operatorname{Ext}_{R \text { Out } H}(V, W)$ is injective in the same way as the corresponding statement of $[31,14.3]$. Suppose we have a non-split short exact sequence of functors

$$
0 \rightarrow S_{H, W} \rightarrow M \rightarrow S_{H, V} \rightarrow 0 .
$$

Evaluating at $H$ this gives a short exact sequence of $R$ Out $(H)$-modules

$$
0 \rightarrow W \rightarrow M(H) \rightarrow V \rightarrow 0
$$

which we show is non-split. If it were to split, the splitting $V \rightarrow M(H)$ would extend uniquely to a morphism $\Delta_{H, V} \rightarrow M$ by adjointness, and the image of this morphism must be $S_{H, V}$ since otherwise it would be the whole of $M$, which is not possible since $\Delta_{H, V}(H)=V$. This provides a morphism which splits the original sequence, thus completing the proof of $(2)$.

Part (3) is proved by a dual argument to (4) and we only give the proof of (4), which in turn is similar to the proof of the first part of (2). This time assume $H$ is a proper section of $K$. If $\Delta_{H, V}$ has $S_{K, W}$ appearing $r$ times in its second Loewy layer then certainly there exists an short exact sequence $(*)$. On the other hand in any sequence $\left(^{*}\right)$ we have $M(H) \cong V$ and so this isomorphism on $V$ extends uniquely by adjointness to a morphism $\Delta_{H, V} \rightarrow M$ which is an epimorphism since it covers $S_{H, V}$. This forces $S_{K, W}$ to occur at least $r$ times in the second Loewy layer of $\Delta_{H, V}$. We conclude that the maximal possibility for $R$ equals the multiplicity of $S_{K, W}$ in the second Loewy layer of $\Delta_{H, V}$, which proves the result.

We now add to the information given in Theorem 5.10 in one respect.

Proposition 5.11. If $\operatorname{Ext}\left(S_{H, V}, S_{K, W}\right) \neq 0$ then either $H$ lies in the vertex of $S_{K, W}$ or $K$ lies in the vertex of $S_{H, V}$.

Proof. We already know from 5.10 that to have a non-split extension either $H$ must be a section of $K$ or $K$ must be a section of $H$. Suppose 
that $H$ is a section of $K$ and that

$$
0 \rightarrow S_{K, W} \rightarrow M \rightarrow S_{H, V} \rightarrow 0
$$

is a non-split extension. Let $\mathcal{D}$ be the vertex of $S_{H, V}$. If $K$ is not a group in $\mathcal{D}$ then the restriction of the sequence to $\mathcal{D}$ has the form

$$
0 \rightarrow 0 \rightarrow M \downarrow_{\mathcal{D}} \rightarrow S_{H, V} \downarrow_{\mathcal{D}} \rightarrow 0
$$

which is split. Therefore by one of the equivalent conditions listed in [3, Prop. VI.3.6] the original sequence must split, which is a contradiction. Therefore $K$ is a group in $\mathcal{D}$. The argument when $K$ is a section of $H$ is similar.

\section{Projective Functors have a $\Delta$-Filtration}

Our main goal in this section is a proof of Theorem 6.3 which shows that projective functors always have a filtration with factors of the form $\Delta_{H, V}$ where $V$ is a summand of a permutation module. To achieve this we first examine carefully the rank as a free $R$-module of $A_{R}^{\mathcal{X}, \mathcal{Y}}(G, K)$. The first lemma is really an elaboration of Lemma 2.2, and for it we consider pairs of epimorphisms of groups $A \stackrel{\alpha}{\longrightarrow} H \stackrel{\beta}{\longleftarrow} B$ where $A$ is a subgroup of $G$ and $B$ is a subgroup of $K$. Thus $H$ is a common homomorphic image of $A$ and $B$. We will say that two such pairs of epimorphisms $(\alpha, \beta)$ and $(\rho, \sigma)$ are equivalent if there is a commutative diagram

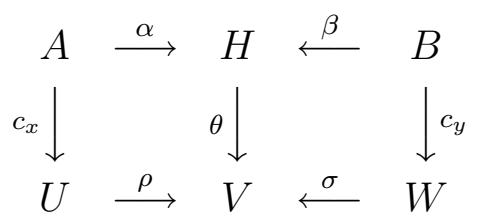

where $c_{x}$ and $c_{y}$ denote conjugation by $x \in G$ and $y \in K, U={ }^{x} A$, $W={ }^{y} B$ and $\theta$ is an isomorphism.

Lemma 6.1. Let $G$ and $K$ be finite groups.

(1) $A_{R}^{\mathcal{X}, \mathcal{Y}}(G, K)$ is a free $R$-module with basis in bijection with the equivalence classes of pairs $(\alpha, \beta)$ of epimorphisms $\alpha: A \rightarrow H$, $\beta: B \rightarrow H$ where $A \leq G, B \leq K, H$ is a common homomorphic image of $A$ and $B$ which is allowed to vary, and such that $\operatorname{Ker} \alpha \in \mathcal{X}$ and $\operatorname{Ker} \beta \in \mathcal{Y}$.

(2) For each fixed group $H, A_{R}^{\mathcal{X}, \mathcal{Y}}(, K)_{(H)}(G)$ is a free $R$-module with basis in bijection with the equivalence classes of pairs $(\alpha, \beta)$ of epimorphisms $\alpha: A \rightarrow H, \beta: B \rightarrow H$ where $A \leq G, B \leq K$, and such that $\operatorname{Ker} \alpha \in \mathcal{X}$ and $\operatorname{Ker} \beta \in \mathcal{Y}$. 
Proof. (1) From its definition, $A_{R}^{\mathcal{X}, \mathcal{Y}}(G, K)$ has as a basis the isomorphism classes of transitive $(G, K)$-bisets with left stabilizers in $\mathcal{X}$ and right stabilizers in $\mathcal{Y}$. By Lemma 2.1 these are precisely the bisets which may be written ${ }_{G} G_{A} \circ{ }_{A} H_{H} \circ{ }_{H} H_{B} \circ{ }_{B} K_{K}$ where $A \leq G, B \leq K$ and $A \stackrel{\alpha}{\longrightarrow} H \stackrel{\beta}{\longleftarrow} B$ are epimorphisms with $\operatorname{Ker} \alpha \in \mathcal{X}$ and $\operatorname{Ker} \beta \in \mathcal{Y}$. By Lemma 2.2, two such bisets are isomorphic if and only if the pairs of epimorphisms are equivalent.

(2) If $\mathcal{E}$ is a set of finite groups closed under taking sections then by Proposition $4.1(3)$ we have that $\left(I_{\mathcal{E}} A_{R}^{\mathcal{X}, \mathcal{Y}}(, K)\right)(G)$ is the span of the transitive bisets which factor through groups in $\mathcal{E}$, namely those corresponding to pairs $A \stackrel{\alpha}{\longrightarrow} J \stackrel{\beta}{\longleftarrow} B$ where $J \in \mathcal{E}$. We see this by observing that if $\gamma: J \rightarrow G$ is a morphism in $\mathcal{C}_{R}^{\mathcal{X}, \mathcal{Y}}$ with $J \in \mathcal{E}$, the corresponding map $A_{R}^{\mathcal{X}, \mathcal{Y}}(J, K) \rightarrow A_{R}^{\mathcal{X}, \mathcal{Y}}(G, K)$ is given by composition with $\gamma$, and such a map has image contained in the span of bisets which factor through groups in $\mathcal{E}$. Equally, every such transitive biset is in the image of such a morphism, by Bouc's Lemma 2.1.

Now $A_{R}^{\mathcal{X}, \mathcal{Y}}(, K)_{\leq H}(G)$ has as a basis the transitive bisets corresponding to pairs $A \stackrel{\alpha}{\longrightarrow} J \stackrel{\beta}{\longleftarrow} B$ where $J$ is a section of $H$, and also $A_{R}^{\mathcal{X}, \mathcal{Y}}(, K)_{<H}(G)$ has as a basis the transitive bisets corresponding to pairs $A \stackrel{\alpha}{\longrightarrow} J \stackrel{\beta}{\longleftarrow} B$ where $J$ is a proper section of $H$. From this the result follows.

Suppose we have two epimorphisms $\alpha: A \rightarrow H$ and $\beta: B \rightarrow H$ with $A \leq G$ and $B \leq K$. As before we put $N_{G}(\alpha)=N_{G}(A) \cap N_{G}(\operatorname{Ker}(\alpha))$ and similarly for $N_{K}(\beta)$, and we regard $\operatorname{Out}(H)$ as a $\left(N_{K}(\beta), N_{G}(\alpha)\right)$ biset where the actions are given by first applying the composite homomorphisms $N_{K}(\beta) \rightarrow \operatorname{Aut}(H) \rightarrow \operatorname{Out}(H)$ and $N_{G}(\alpha) \rightarrow \operatorname{Aut}(H) \rightarrow$ $\operatorname{Out}(H)$, followed by left and right multiplication, respectively.

Lemma 6.2. Given finite groups $G, H$ and $K$, the number of equivalence classes of pairs $(\alpha, \beta)$ of epimorphisms with a fixed codomain $H$, $\operatorname{Ker} \alpha \in \mathcal{X}$ and $\operatorname{Ker} \beta \in \mathcal{Y}$ equals

$$
\sum_{\alpha \in\left[\operatorname{Aut} H \backslash \operatorname{Sec}_{\mathcal{X}}(G, H) / G\right]} \sum_{\beta \in\left[\operatorname{Aut} H \backslash \operatorname{Sec} \mathcal{Y}_{(K, H) / K]}\right.}\left|N_{K}(\beta) \backslash \operatorname{Out}(H) / N_{G}(\alpha)\right| .
$$

This number equals

$$
\operatorname{rank}_{R} A_{R}^{\mathcal{X}, \mathcal{Y}}(, K)_{(H)}(G)
$$

Proof. The pairs of epimorphisms $A \stackrel{\alpha}{\longrightarrow} H \stackrel{\beta}{\longleftarrow} B$ biject with the set $\Omega=$ $\operatorname{Sec}_{\mathcal{X}}(G, H) \times \operatorname{Sec}_{\mathcal{Y}}(K, H)$, and the equivalence classes of such pairs are the orbits of the action of Aut $H \times G \times K$ on this set given by 
$(\phi, g, k)(\alpha, \beta)=\left(\phi \alpha c_{g}^{-1}, \phi \beta c_{k}^{-1}\right)$. There is in fact an action of Aut $H \times$ $G \times$ Aut $H \times K$ given by $(\phi, g, \psi, k)(\alpha, \beta)=\left(\phi \alpha c_{g}^{-1}, \psi \beta c_{k}^{-1}\right)$ and the orbits on $\Omega$ under this action are unions of the orbits under the action of Aut $H \times G \times K$, since this latter group acts as a subgroup of the bigger group with Aut $H$ embedded diagonally in Aut $H \times$ Aut $H$.

Consider now two elements $(\alpha, \beta)$ and $\left(\phi \alpha c_{g}^{-1}, \psi \beta c_{k}^{-1}\right)$ in the same Aut $H \times G \times$ Aut $H \times K$-orbit. The second element lies in the same Aut $H \times G \times K$-orbit as $\left(\psi^{-1} \phi \alpha, \beta\right)$ so that the elements $(\gamma \alpha, \beta)$ with $\gamma \in$ Aut $H$ contain a complete set of representatives of the Aut $H \times G \times K$ orbits on the Aut $H \times G \times$ Aut $H \times K$-orbit containing $(\alpha, \beta)$. Two elements $\left(\gamma_{1} \alpha, \beta\right)$ and $\left(\gamma_{2} \alpha, \beta\right)$ lie in the same Aut $H \times G \times K$-orbit if and only if $\left(\gamma_{2} \alpha, \beta\right)=\left(\phi \gamma_{1} \alpha c_{x}^{-1}, \phi \beta c_{y}^{-1}\right)$ for certain $x \in G$ and $y \in K$, which entails $x \in N_{G}(\alpha), y \in N_{G}(\beta), \phi=c_{\beta(y)}$ and $\gamma_{2}^{-1} \phi \gamma_{1}=c_{\alpha(x)}$, so that $\gamma_{2}=c_{\beta(y)} \gamma_{1} c_{\alpha(x)}^{-1}$. Thus the condition that $\left(\gamma_{1} \alpha, \beta\right)$ and $\left(\gamma_{2} \alpha, \beta\right)$ lie in the same Aut $H \times G \times K$-orbit is that $\gamma_{1}$ and $\gamma_{2}$ represent the same double coset in $N_{K}(\beta) \backslash$ Aut $H / N_{G}(\alpha)$. These double cosets biject with the double cosets $N_{K}(\beta) \backslash$ Out $H / N_{G}(\alpha)$ since Inn $H$ is contained in the image of both $N_{G}(\alpha)$ and $N_{K}(\beta)$ in Aut $H$.

Since the Aut $H \times G \times$ Aut $H \times K$-orbits on $\operatorname{Sec}_{\mathcal{X}}(G, H) \times \operatorname{Sec}_{\mathcal{Y}}(K, H)$ are

$$
\left(\text { Aut } H \backslash \operatorname{Sec}_{\mathcal{X}}(G, H) / G\right) \times\left(\operatorname{Aut} H \backslash \operatorname{Sec}_{\mathcal{Y}}(K, H) / K\right),
$$

and each consists of $\mid N_{K}(\beta) \backslash$ Out $H / N_{G}(\alpha) \mid$ orbits of Aut $H \times G \times K$, the first statement follows.

The second statement is part (2) of Lemma 6.1.

Theorem 6.3. Let $R$ be a commutative ring with 1 , let $1=H_{1}, H_{2}, \ldots$ be a list of groups in which the sections of each group $H_{i}$ always appear earlier than $H_{i}$ and let $P$ be a projective globally defined Mackey functor. Let $P_{r}=I_{\left\{H_{1}, \ldots, H_{r}\right\}} P$ be the terms of the corresponding ascending filtration of $P$. Then

$$
P_{r} / P_{r-1} \cong P_{\left(H_{r}\right)} \cong \Delta_{H_{r}, \bar{P}\left(H_{r}\right)} .
$$

In this formula $\bar{P}\left(H_{r}\right)$ is a direct summand of a permutation $R \operatorname{Out}\left(H_{r}\right)$ module.

Proof. Step 1. We claim that it suffices to consider the case when $P=$ $A_{R}^{\mathcal{X}, \mathcal{Y}}(, K)$ is a representable functor. This is because an arbitrary projective $P$ is a direct summand of a direct sum of such functors (see [32] or [7]) and the assertion of the theorem for a class of functors implies the same assertion for direct sums and direct summands of functors in the class. 
Step 2. For each pair of groups $H$ and $G$ we examine the module $A_{R}^{\mathcal{X}, \mathcal{Y}}(, K)_{(H)}(G)$ as an $R \operatorname{Out}(G)$-module, being particularly interested in the case $G=H$. It is the permutation module on equivalence classes of pairs $(\alpha, \beta)$ as in Lemma 6.1 part 2. The action of $\nu \in$ Aut $G$ is given by $\nu(\alpha, \beta)=\left(\alpha \nu^{-1}, \beta\right)$. At this point we have proven that for the artbirary projective $P$ the module $\bar{P}\left(H_{r}\right)$ is a direct summand of a permutation module in view of Corollary 4.5.

Examining the action of $\nu \in$ Aut $G$ further, $\nu(\alpha, \beta) \sim(\alpha, \beta)$ if and only if $\left(\alpha \nu^{-1}, \beta\right)=\left(\phi \alpha c_{g}^{-1}, \phi \beta c_{k}^{-1}\right)$ for some $\phi \in$ Aut $H, k \in K, g \in G$, and this happens if and only if $\phi$ lies in the image of $N_{K}(\beta)$ in $\operatorname{Aut}(H)$ and $\alpha \nu^{-1}=\phi \alpha c_{g}^{-1}$. In determining the action of Out $G$ we may replace $\nu$ by $c_{g}^{-1} \nu$ since these represent the same element of Out $G$, and the effect of this is that we may assume that $g=1$. Now the condition for $\nu(\alpha, \beta) \sim(\alpha, \beta)$ is that $\nu \in \operatorname{Stab}_{\text {Aut } G} \alpha$, and $\nu$ induces $\phi^{-1} \in N_{K}(\beta)$ on $H$. When $G=H$ this simplifies, and we have

$$
A_{R}^{\mathcal{X}, \mathcal{Y}}(, K)_{(H)}(H)=\bigoplus_{\beta \in\left[\text { Aut } H \backslash \operatorname{Sec}_{\mathcal{Y}}(K, H) / K\right]} R \uparrow_{N_{K}(\beta)}^{\text {Out } H} .
$$

In this formula and what follows we write simply $N_{K}(\beta)$ instead of the image of $N_{K}(\beta)$ in $\operatorname{Out}(H)$. The formula will be used in Step 5 .

Step 3. We claim that to show that $P_{r} / P_{r-1} \cong P_{\left(H_{r}\right)} \cong \Delta_{H_{r}, \bar{P}\left(H_{r}\right)}$ it suffices to prove the result when $R=\mathbb{Z}$. By step 1 we assume that $P=A_{R}^{\mathcal{X}, \mathcal{Y}}(, K)$ and from the definition we have $A_{R}^{\mathcal{X}, \mathcal{Y}}(, K)=$ $R \otimes_{\mathbb{Z}} A_{\mathbb{Z}}^{\mathcal{X}, \mathcal{Y}}(, K)$. Assuming that $A_{\mathbb{Z}}^{\mathcal{X}, \mathcal{Y}}(, K)$ has an ascending filtration with $\Delta$ factors as claimed, all terms in the ascending filtration of $A_{\mathbb{Z}}(, K)$ have values which are free abelian groups, so that on tensoring with $R$ we obtain a filtration of $A_{R}^{\mathcal{X}, \mathcal{Y}}(, K)$ with $\Delta$-factors of the required form, since these factors behave well on extending the ground ring from $\mathbb{Z}$ to $R$.

Step 4. We claim that the result will follow when $R=\mathbb{Z}$ if we can prove it when $R$ is a field. Over $\mathbb{Z}$ we wish to show that each of the epimorphisms $\Delta_{H_{r}, \bar{P}\left(H_{r}\right)} \rightarrow P_{r} / P_{r-1}$ is an isomorphism, or in other words that it has zero kernel. All evaluations of these functors are free abelian groups, so the epimorphisms are split at each evaluation, and so they will be isomorphisms precisely if after tensoring with any field they are isomorphisms. 
Step 5. Let us write $U=A_{R}^{\mathcal{X}, \mathcal{Y}}(, K)_{(H)}(H)$ and compute $\operatorname{rank}_{R} \Delta_{H, U}(G)$. Using the formula

$$
\begin{aligned}
\Delta_{H, U}(G) & =\bigoplus_{\alpha \in\left[\operatorname{Aut} H \backslash \operatorname{Sec}_{\mathcal{X}}(G, H) / G\right]}{ }^{\alpha} U^{N_{K}(\alpha)} \\
& =\bigoplus_{\alpha \in\left[\operatorname{Aut~} H \backslash \operatorname{Sec}_{\mathcal{X}}(G, H) / G\right]} \bigoplus_{\beta \in\left[\operatorname{Aut} H \backslash \operatorname{Sec}_{\mathcal{Y}}(K, H) / K\right]}\left(R \uparrow_{N_{K}(\beta)}^{\text {Out } H}\right)^{N_{K}(\alpha)}
\end{aligned}
$$

which comes from substituting the result of Step 2, and the fact that

$$
\operatorname{rank}_{R}\left(R \uparrow_{N_{K}(\beta)}^{\mathrm{Out} H}\right)^{N_{K}(\alpha)}=\mid N_{K}(\alpha) \backslash \text { Out } H / N_{K}(\beta) \mid
$$

we see that

$$
\begin{aligned}
& \operatorname{rank}_{R} \Delta_{H, U}(G) \\
& =\sum_{\alpha \in\left[\operatorname{Aut} H \backslash \operatorname{Sec}_{\mathcal{X}}(G, H) / G\right]} \sum_{\beta \in\left[\operatorname{Aut} H \backslash \operatorname{Sec} \mathcal{Y}_{(K, H) / K]}\right.} \mid N_{K}(\alpha) \backslash \text { Out } H / N_{K}(\beta) \mid \\
& =\operatorname{rank}_{R} A_{R}^{\mathcal{X}, \mathcal{Y}}(, K)_{(H)}(G)
\end{aligned}
$$

by Lemma 6.2. It follows that for each $r$ the epimorphism $\Delta_{H_{r}, \bar{P}\left(H_{r}\right)} \rightarrow$ $P_{r} / P_{r-1}$ given by Corollary 5.7 is an isomorphism, since on evaluation at each group $G$ the two modules have the same dimension.

We can describe the values of the functors $P_{(H)}$ which are the factors in the ascending filtration of a projective functor $P$ more explicitly in one particular case.

Proposition 6.4. Let $V$ be a simple $R$ Out $H$-module where $R$ is a field or a local ring. Then $\overline{P_{H, V}}(H)=\left(P_{H, V}\right)_{(H)}(H)$ is the projective cover $P_{V}$ of $V$ as an $R$ Out $H$-module.

Proof. Putting $n_{K, W}(H)=\operatorname{dim} S_{K, W}(H) / \operatorname{dim} \operatorname{End}(W)$ we have by the argument of $[32,5.6]$ that $A_{R}^{\mathcal{X}, \mathcal{Y}}(, H) \cong \bigoplus_{K, W} P_{K, W}^{n_{K, W}(H)}$ and so using Lemma 6.1(2) for the first of the following isomorphisms,

$$
R \text { Out } H \cong A_{R}^{\mathcal{X}, \mathcal{Y}}(, H)_{(H)}(H) \cong \bigoplus_{\text {simple } R \text { Out } H-\text { modules } V}\left(P_{H, V}\right)_{(H)}(H)^{n_{H, V}(H)} .
$$

For the second isomorphism we note that $n_{K, W}(H)=0$ unless $K$ is a section of $H$, and for such $K,\left(P_{K, W}\right)_{(H)}=0$ unless $K \cong H$ since $P_{K, W}$ is generated by its value at $K$. None of the summands in the direct sum is zero (since $P_{H, V}$ is not generated by its values on proper sections of $H$ ), and their multiplicities $n_{H, V}(H)=\operatorname{dim} V / \operatorname{dim} \operatorname{End}(V)$ equal the multiplicities of the indecomposable summands of $R$ Out $H$. Thus the summands in the direct sum are exactly the indecomposable summands of $R$ Out $H$, namely the projective covers of the simple modules. The 
fact that $\left(P_{H, V}\right)_{(H)}(H)$ has $V$ as an image identifies $\left(P_{H, V}\right)_{(H)}(H)$ with $P_{V}$.

We now identify the standard and proper standard quotients of projectives associated to the preorder on the set which parametrizes the simple functors. This preorder is $(K, W) \leq(H, V)$ if and only if $H$ is a section of $K$. There are dual statements concerning the functors $\nabla_{H, P_{V}}, \nabla_{H, V}$ in their role as costandard and proper costandard subobjects of the indecomposable injectives $I_{H, V}$. We leave these for the reader to formulate.

Corollary 6.5. Let $R$ be a field or a local ring.

(1) $\Delta_{H, P_{V}}$ is the largest quotient of $P_{H, V}$ all of whose composition factors have the form $S_{K, W}$ where $(K, W) \leq(H, V)$ (that is, where $H$ is a section of $K$ ).

(2) $\Delta_{H, V}$ is the largest quotient of $P_{H, V}$ whose radical only has composition factors of the form $S_{K, W}$ where $(K, W)<(H, V)$ (or in other words, where $H$ is a proper section of $K)$.

Proof. (1) By Theorem 6.3 and Proposition 6.4 there is a short exact sequence $0 \rightarrow M \rightarrow P_{H, V} \rightarrow \Delta_{H, P_{V}} \rightarrow 0$ where $M$ has a filtration with factors $\Delta_{J, U}$ where $J$ is a proper section of $H$, and it follows that $M$ is generated by its values on proper sections of $H$. From this it follows that any larger quotient of $P_{H, V}$ than $\Delta_{H, P_{V}}$ must have a composition factor which is non-zero on a proper section of $H$, and from this the result follows.

(2) We will show that $\Delta_{H, V}$ is the largest quotient of $\Delta_{H, P_{V}}$ all of whose composition factors are $S_{K, W}$ where $(K, W)<(H, V)$, except for the top composition factor $S_{H, V}$. This will suffice to prove the result since the largest quotient of $P_{H, V}$ with the same property is certainly a quotient of $\Delta_{H, P_{V}}$, by part (1).

We consider the short exact sequence of $R$ Out $H$-modules $0 \rightarrow L \rightarrow$ $P_{V} \rightarrow V \rightarrow 0$ where $L$ is the radical of $P_{V}$ and apply one of the functors of Proposition 5.2 to get a sequence

$$
\Delta_{H, L} \rightarrow \Delta_{H, P_{V}} \rightarrow \Delta_{H, V} \rightarrow 0 .
$$

This sequence is exact since the functor we have applied is a left adjoint. Now $\Delta_{H, L}$ is generated by its value at $H$, and from this it follows that any quotient of $\Delta_{H, P_{V}}$ larger than $\Delta_{H, V}$ must have a composition factor which is non-zero at $H$. This implies the result.

The identification of standard and costandard objects has some immediate consequences to do with the values of Hom and Ext between $\Delta$ and $\nabla$ functors which are listed in [36, Prop. 3.1], for example. In 
our particular situation the general formulas which appear there can be improved, and we present the stronger versions here.

Proposition 6.6. Let $H$ and $K$ be finite groups, let $V$ be a $R$ Out $H$ module let $W$ be an $R$ Out $K$-module and let $M$ be a globally defined Mackey functor. We have

(1) $\operatorname{Hom}\left(\Delta_{H, V}, M\right)=0$ and $\operatorname{Hom}\left(M, \nabla_{H, V}\right)=0$ unless $M$ is nonzero on a section of $H$. In particular, $\operatorname{Hom}\left(\Delta_{H, V}, \Delta_{K, W}\right)=0$ and $\operatorname{Hom}\left(\nabla_{K, W}, \nabla_{H, V}\right)=0$ unless $K$ is a section of $H$.

$$
\operatorname{Hom}\left(\Delta_{H, V}, \nabla_{K, W}\right)= \begin{cases}\operatorname{Hom}_{R \text { Out } H}(V, W) & \text { if } H=K, \\ 0 & \text { if } H \neq K,\end{cases}
$$

the isomorphism in case $H=K$ being induced by evaluation at $H$.

(3) For any $M$, if either $\operatorname{Ext}^{1}\left(\Delta_{H, V}, M\right) \neq 0$ or $\operatorname{Ext}^{1}\left(M, \nabla_{H, V}\right) \neq$ 0 then $M$ is non-zero on some section of $H$; if $V$ is further assumed to be projective then $M$ must be non-zero on some proper section of $H$.

(4)

$$
\operatorname{Ext}^{1}\left(\Delta_{H, V}, \nabla_{K, W}\right)= \begin{cases}\operatorname{Ext}_{R \text { Out } H}^{1}(V, W) & \text { if } H=K, \\ 0 & \text { if } H \neq K .\end{cases}
$$

Proof. (1) and (2) are immediate from the adjoint properties of $\Delta$ and $\nabla$ expressed in Proposition 5.2, together with the fact that $\Delta_{H, V}$ and $\nabla_{H, V}$ are only non-zero on groups which have $H$ as a section.

For (3) and (4) we calculate $\operatorname{Ext}^{1}\left(\Delta_{H, V}, \nabla_{K, W}\right)$ using the start of a projective resolution which appears as the top row of the following diagram:

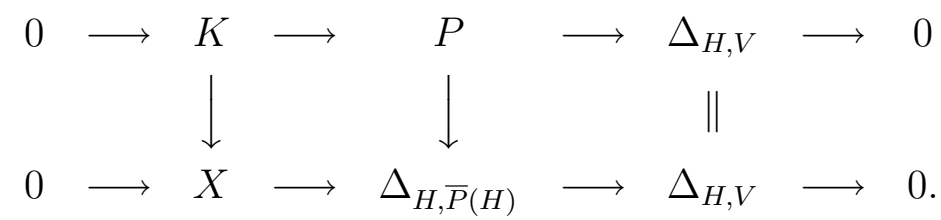

Here $P$ is a projective globally defined Mackey functor which we take to be generated at $H$ since $\Delta_{H, V}$ is generated at $H$. Now by Theorem 6.3 $P$ has a $\Delta$-filtration whose top term is $I_{<H} P$. The map $P \rightarrow \Delta_{H, V}$ vanishes on $I_{<H} P$ which is generated on proper sections of $H$, so it factors as $P \rightarrow P_{(H)} \rightarrow \Delta_{H, V}$, and by Theorem 6.3 and Proposition 6.4 $P_{(H)} \cong \Delta_{H, \bar{P}(H)}$ where $\bar{P}(H)$ is a projective $R$ Out $H$-module. This explains the middle vertical morphism. We define $X$ and $K$ in this diagram to be the kernels of the horizontal maps on the right. We see from this diagram that the map $K \rightarrow X$ is an epimorphism, and 
in fact its kernel has a $\Delta$-filtration with factors generated at proper sections of $H$. Consider the short exact sequence of $R$ Out $H$-modules $0 \rightarrow I \rightarrow \bar{P}(H) \rightarrow V \rightarrow 0$ where $I$ is defined as the kernel. Since the functor of Proposition 5.2 is a left adjoint we deduce that $\Delta_{H, I} \rightarrow$ $\Delta_{H, \bar{P}(H)} \rightarrow \Delta_{H, V} \rightarrow 0$ is exact, and from this we deduce that $X$ is an image of $\Delta_{H, I}$ and so is generated at $H$.

We compute Ext by an exact sequence

$$
\operatorname{Hom}(P, M) \rightarrow \operatorname{Hom}(K, M) \rightarrow \operatorname{Ext}\left(\Delta_{H, V}, M\right) \rightarrow 0 .
$$

Since $K$ has a filtration with factors which are generated on sections of $H$, we deduce that $\operatorname{Hom}(K, M)$ is zero unless $M$ is non-zero on a section of $H$, and this proves the very first part of (3). If $V=\bigoplus P_{V_{i}}$ is projective then we could have chosen $P=\bigoplus P_{H, V_{i}}$ so that $X=0$ and $K$ is generated by its values on proper sections of $H$. We deduce that $\operatorname{Hom}(K, M)$ is zero unless $M$ is non-zero on a proper section of $H$, and this proves the second statement of (3) in the case of $\Delta_{H, V}$. The parts of (3) about $\nabla_{H, V}$ follow by duality.

In the situation of (4), we deduce from (3) that the Ext group is zero unless both $H$ is a section of $K$ and $K$ is a section of $H$, which forces $H \cong K$. In case $H=K$ with $M=\nabla_{H, W}$, since the kernel of $K \rightarrow X$ is generated on proper sections of $H$ any homomorphism $K \rightarrow \nabla_{H, W}$ must vanish on that kernel. We deduce that the sequence which computes Ext identifies as

$$
\operatorname{Hom}\left(\Delta_{H, \bar{P}(H)}, \nabla_{H, W}\right) \rightarrow \operatorname{Hom}\left(X, \nabla_{H, W}\right) \rightarrow \operatorname{Ext}\left(\Delta_{H, V}, \nabla_{H, W}\right) \rightarrow 0 .
$$

The morphism $\Delta_{H, I} \rightarrow X$ previously described is an isomorphism on evaluation at $H$, and so its kernel is generated at groups which have $H$ as a proper section. It follows from the adjoint property of $\nabla$ that there is no non-zero homomorphism from this kernel to $\nabla_{H, W}$ and so $\operatorname{Hom}\left(X, \nabla_{H, W}\right) \rightarrow \operatorname{Hom}\left(\Delta_{H, I}, \nabla_{H, W}\right)$ is an isomorphism. Furthermore by the adjoint properties of $\Delta$ and $\nabla$ we have $\operatorname{Hom}\left(\Delta_{H, I}, \nabla_{H, W}\right) \cong$ $\operatorname{Hom}_{R \text { Out } H}(I, W)$ and $\operatorname{Hom}\left(\Delta_{H, \bar{P}(H)}, \nabla_{H, W}\right) \cong \operatorname{Hom}_{R \text { Out } H}(\bar{P}(H), W)$. We deduce that the sequence which computes Ext identifies as

$\operatorname{Hom}_{R \text { Out } H}(\bar{P}(H), W) \rightarrow \operatorname{Hom}_{R \text { Out } H}(I, W) \rightarrow \operatorname{Ext}\left(\Delta_{H, V}, \nabla_{H, W}\right) \rightarrow 0$.

Since this sequence also computes $\operatorname{Ext}_{R \text { Out } H}(V, W)$ this completes the proof.

We comment that in the situation with $H=K$ in part (4) of the above result, the isomorphism of Ext groups is induced by evaluation at $H$, in the sense that an extension of globally defined Mackey functors on evaluation at $H$ gives an extension of $R$ Out $H$-modules. 


\section{Highest Weight CAtegories of Globally Defined Mackey FUNCTORS}

We start this section with the proof of Theorem 7.2 which states that when $R$ is a field of characteristic zero, $\operatorname{Mack}_{R}^{\mathcal{X}, \mathcal{Y}}(\mathcal{D})$ is a highest weight category (except that we fail to verify one of the finiteness conditions when $\mathcal{D}$ is infinite). After that we show that restriction of functors from $\mathcal{D}$ to a smaller class $\mathcal{E}$ preserves the highest weight structure.

Recall from the introduction that we put a partial order on the set

$$
\begin{aligned}
\Lambda=\{(H, V) \mid & H \text { is a group, } \\
& V \text { is a simple } R \text { Out } H \text {-module, } \\
& \text { both taken up to isomorphism }\}
\end{aligned}
$$

which is slightly, but importantly, different to the preorder considered at the end of the last section. The partial order is determined by putting $(H, V)>(K, W)$ if and only if $H$ is a proper section of $K$. The point is that with this partial order, pairs $(H, V)$ and $(H, W)$ are only comparable if $V \cong W$, which was not the case with the preorder. It is also possible to work with a partial order which refines this one, such as that determined by $(H, V)>(K, W)$ if and only if $|H|<|K|$. The statements of results and the proofs remain the same.

We start by stating the essential features which make a highest weight category, ignoring for the moment the finiteness conditions.

Proposition 7.1. Let $R$ be a field, $H$ a group and $V$ a simple $R$ Out $H$-module.

(1) The functor $\Delta_{H, V}$ has a unique simple quotient and $\nabla_{H, V}$ has a unique simple submodule, each isomorphic to $S_{H, V}$. Moreover, all the other composition factors of $\Delta_{H, V}$ and $\nabla_{H, V}$ have the form $S_{K, W}$ with $(K, W)<(H, V)$. Each composition factor occurs with finite multiplicity, and we have

$$
\left[\Delta_{H, V}: S_{K, W}\right]=\left[\nabla_{H, V^{*}}: S_{K, W^{*}}\right] .
$$

(2) Assuming $R$ has characteristic zero, the indecomposable projective $P_{H, V}$ has a finite length filtration in which the top factor is isomorphic to $\Delta_{H, V}$, the remaining factors being isomorphic to $\Delta_{K, W}$ with $W$ simple and $(H, V)<(K, W)$.

(3) Assuming $R$ has characteristic zero, the indecomposable injective $I_{H, V}$ has a finite length filtration in which the bottom factor is isomorphic to $\nabla_{H, V}$, the remaining factors being isomorphic to $\nabla_{K, W}$ with $W$ simple and $(H, V)<(K, W)$. 
Proof. The statements for the $\nabla_{H, V}$ follow by duality from the corresponding statements for the $\Delta_{H, V}$ using Corollary 5.9 and Corollary 4.4, and so we only give proofs for the $\Delta_{H, V}$.

(1) We have seen in Corollary 5.5 that $\Delta_{H, V}$ has a unique simple quotient $S_{H, V}$. Since $\Delta_{H, V}(H)=S_{H, V}(H)=V$ the composition factor $S_{H, V}$ occurs in $\Delta_{H, V}$ with multiplicity one, and because $\Delta_{H, V}(K)=0$ unless $H$ is a section of $K$, all other composition factors $S_{K, W}$ of $\Delta_{H, V}$ have $(K, W)<(H, V)$. Each composition factor multiplicity $\left[\Delta_{H, V}\right.$ : $\left.S_{K, W}\right]$ is finite since it is at most the multiplicity $\left[\Delta_{H, V}(K): W\right]$ and $\operatorname{dim} \Delta_{H, V}(K)$ is finite.

(2) Since $P_{H, V}$ is generated by its value at $H$ we have $P_{H, V}=$ $\left(P_{H, V}\right)_{\leq H}$, and on arranging the sections of $H$ in some order of nondecreasing size $1=H_{1}, \ldots, H_{n}=H$ we obtain a filtration of $P_{H, V}$ in which the factors are $\left(P_{H, V}\right)_{\left(H_{r}\right)}=\Delta_{H_{r}, \overline{P_{H, V}}\left(H_{r}\right)}$ by Theorem 6.3. Since $R$ is a field of characteristic zero we may write $\overline{P_{H, V}}\left(H_{r}\right)=U_{1} \oplus \cdots \oplus U_{t}$, a direct sum of simple $R$ Out $H_{r}$-modules, and now

$$
\Delta_{H_{r}, \overline{P_{H, V}}\left(H_{r}\right)} \cong \Delta_{H_{r}, U_{1}} \oplus \cdots \oplus \Delta_{H_{r}, U_{t}},
$$

a finite direct sum. Thus $P_{H, V}$ is filtered by $\Delta_{K, W}$ with $(K, W)>$ $(H, V)$, except for the factor $\left(P_{H, V}\right)_{(H)}$, and each isomorphism type of $\Delta_{K, W}$ appears only finitely many times. We saw in Proposition 6.4 that $\left(P_{H, V}\right)_{(H)}(H)=V$ - the projective cover of $V$ here - so that $\left(P_{H, V}\right)_{(H)} \cong \Delta_{H, V}$. Thus $\Delta_{H, V}$ occurs precisely once, at the top of the filtration.

We come now to one of the main results of this paper.

Theorem 7.2. Let $R$ be a field of characteristic zero. With respect to the partial order on the simple objects defined above, and when $\mathcal{D}$ is finite, $\operatorname{Mack}_{R}^{\mathcal{X}, \mathcal{Y}}(\mathcal{D})$ is a highest weight category. When $\mathcal{D}$ is not finite, $\operatorname{Mack}_{R}^{\mathcal{X}, \mathcal{Y}}(\mathcal{D})$ is still a highest weight category, except that indecomposable injectives might conceivably not be unions of their subobjects of finite length.

Proof. We verify the conditions for $\operatorname{Mack}_{R}^{\mathcal{X}, \mathcal{Y}}(\mathcal{D})$ to be a highest weight category given in Definition 3.1 of [16]. In view of Proposition 7.1 we only need to consider the various finiteness conditions which are required.

When $\mathcal{D}$ is finite the global Mackey algebra $\mu_{R}^{\mathcal{X}, \mathcal{Y}}(\mathcal{D})$ is finite dimensional and the finiteness conditions mentioned in 3.1 of [16] are automatically satisfied. In this case the proof is complete.

When $\mathcal{D}$ contains infinitely many isomorphism classes of groups, most of the remaining conditions in the definition of a highest weight 
category are straightforward to check. The poset $\Lambda$ is clearly intervalfinite; and the Grothendieck condition $B \cap \bigcup A_{\alpha}=\bigcup\left(B \cap A_{\alpha}\right)$ for subobjects $B$ and $A_{\alpha}$ of an object $X$ holds because globally defined Mackey functors are modules for an algebra. The various $I_{H, V}$ provide enough injectives. The most complicated thing to verify is that the $I_{H, V}$ and the $\nabla_{H, V}$ are the union of their subobjects of finite length. We leave this condition unverified.

We now show that the highest weight structure behaves well under restriction and induction between section closed sets of finite groups $\mathcal{E} \subseteq \mathcal{D}$. We have already seen in Proposition 3.1 that a simple functor on $\mathcal{D}$ restricts to a simple functor with the same parametrization or zero, and in Proposition 3.3 and Corollary 5.4 we have seen that indecomposable projective and $\Delta$ functors restrict and induce to each other, preserving the parametrization. By duality (over a field), the same thing is true of the indecomposable injective and $\nabla$ functors. It follows from this that when $H$ is a group in $\mathcal{E}$ the composition factor multiplicites of the $S_{H, V}$ and the filtration multiplicites of the $\Delta_{H, V}$ in the $P_{H, V}$ may be computed by first restricting to $\mathcal{E}$. We can in fact say more than this.

Proposition 7.3. Let $R$ be a field of characteristic zero, let $\mathcal{E} \subseteq \mathcal{D}$ be section-closed sets of finite groups and let ${ }^{\mathcal{D}} P_{H, V}$ be an indecomposable projective object of $\operatorname{Mack}_{R}^{\mathcal{X}, \mathcal{Y}}(\mathcal{D})$, where $H$ is a group in $\mathcal{E}$. Suppose that

$$
0=P_{r} \subseteq P_{r-1} \subseteq \cdots \subseteq P_{1} \subseteq P_{0}=P_{H, V}^{\mathcal{D}}
$$

is a filtration of ${ }^{\mathcal{D}} P_{H, V}$ with $\Delta$ factors. Then on restriction to $\operatorname{Mack}_{R}^{\mathcal{X}, \mathcal{Y}}(\mathcal{E})$ the terms $P_{i} \downarrow_{\mathcal{E}}^{\mathcal{D}}$ are a filtration of ${ }^{\mathcal{E}} P_{H, V}$ with factors ${ }^{\mathcal{D}} \Delta_{K, W} \downarrow_{\mathcal{E}}^{\mathcal{D}}=$ ${ }^{\mathcal{E}} \Delta_{K, W}$, where the left superscripts $\mathcal{D}$ and $\mathcal{E}$ indicate the domain of definition of these functors. The subfunctors $P_{i}$ are all projective relative to $\mathcal{E}$ so that we have $P_{i} \cong P_{i} \downarrow_{\mathcal{E}}^{\mathcal{D}} \uparrow_{\mathcal{E}}^{\mathcal{D}}$. We have an equality of filtration multiplicities:

$$
\left[{ }^{\mathcal{D}} P_{H, V}:{ }^{\mathcal{D}} \Delta_{K, W}\right]=\left[{ }^{\mathcal{E}} P_{H, V}:{ }^{\mathcal{E}} \Delta_{K, W}\right] .
$$

Similar statements hold for filtrations of injective functors with $\nabla$ factors and the corresponding filtration multiplicities.

Proof. The initial statements about restricting the filtration are clear since restriction is an exact functor and by Corollary 5.4 the $\Delta$ factors restrict correctly. The statement about filtration multiplicities is a consequence. 
To prove projectivity relative to $\mathcal{E}$ of the $P_{i}$, consider the short exact sequences $0 \rightarrow P_{i+1} \downarrow_{\mathcal{E}}^{\mathcal{D}} \rightarrow P_{i} \downarrow_{\mathcal{E}}^{\mathcal{D}} \rightarrow\left(P_{i} / P_{i+1}\right) \downarrow_{\mathcal{E}}^{\mathcal{D}} \rightarrow 0$ and apply induction $\uparrow_{\mathcal{E}}^{\mathcal{D}}$, which is right exact. We deduce that on evaluation at each group $G$ in $\mathcal{D}$,

$$
\operatorname{dim} P_{i} \downarrow_{\mathcal{E}}^{\mathcal{D}} \uparrow_{\mathcal{E}}^{\mathcal{D}}(G)-\operatorname{dim} P_{i+1} \downarrow_{\mathcal{E}}^{\mathcal{D}} \uparrow_{\mathcal{E}}^{\mathcal{D}}(G) \leq \operatorname{dim}\left(P_{i} / P_{i+1}\right) \downarrow_{\mathcal{E}}^{\mathcal{D}} \uparrow_{\mathcal{E}}^{\mathcal{D}}(G)
$$

with equality for all $G$ if and only if $\operatorname{dim} P_{i+1} \downarrow_{\mathcal{E}}^{\mathcal{D}} \uparrow_{\mathcal{E}}^{\mathcal{D}} \rightarrow \operatorname{dim} P_{i} \downarrow_{\mathcal{E}}^{\mathcal{D}} \uparrow_{\mathcal{E}}^{\mathcal{D}}$ is a monomorphism. Summing these equalities we obtain

$$
\operatorname{dim} P_{H, V}(G)=\operatorname{dim} P_{H, V} \downarrow_{\mathcal{E}}^{\mathcal{D} \uparrow} \uparrow_{\mathcal{E}}^{\mathcal{D}}(G) \leq \sum_{i=0}^{r-1} \operatorname{dim}\left(P_{i} / P_{i+1}\right) \downarrow_{\mathcal{E}}^{\mathcal{D}} \uparrow_{\mathcal{E}}^{\mathcal{D}}(G)
$$

with equality for all $G$ if and only if the $\operatorname{dim} P_{i} \downarrow_{\mathcal{E}}^{\mathcal{D}} \uparrow_{\mathcal{E}}^{\mathcal{D}}$ form a filtration of $P_{H, V}$. The factors $P_{i} / P_{i+1}$ all have the form $\Delta_{K, W}$ and we know that the natural counit map $\Delta_{K, W} \downarrow_{\mathcal{E}}^{\mathcal{D}} \uparrow_{\mathcal{E}}^{\mathcal{D}} \rightarrow \Delta_{K, W}$ is an isomorphism by Corollary 5.4. Thus the inequality of dimensions is indeed an equality, since the terms in the sum are the dimensions of the factors in the original filtration of $P_{H, V}$. We deduce that the $P_{i} \downarrow_{\mathcal{E}}^{\mathcal{D}} \uparrow_{\mathcal{E}}^{\mathcal{D}}$ form a filtration of $P_{H, V}$ isomorphic by the natural counit map to the original filtration, and in particular $P_{i} \cong P_{i} \downarrow_{\mathcal{E}}^{\mathcal{D}} \uparrow_{\mathcal{E}}^{\mathcal{D}}$.

\section{The Cartan matrix of Globally Defined Mackey FUNCTORS}

In this section we prove when $R$ is a field that the Cartan matrix of $\operatorname{Mack}_{R}^{\mathcal{X}, \mathcal{Y}}(\mathcal{D})$ is non-singular in many circumstances, and that when $\mathcal{X}=\mathcal{Y}$ and $R$ is sufficiently large it is symmetric. Here, when we say that $R$ is sufficiently large we will mean that $R$ is a splitting field for Out $H$, for all $H$ in $\mathcal{D}$.

We start by showing symmetry of the Cartan matrix in case $R$ is a sufficiently large field of characteristic zero. It follows from Proposition 7.3 that to compute the numbers

$$
c_{(H, V),(K, W)}=\left[P_{K, W}, S_{H, V}\right]
$$

which are the entries in the Cartan matrix it suffices to assume $\mathcal{D}$ is a section-closed set of groups which contains $K$ and $H$, and so for such a computation we may assume $\mathcal{D}$ is finite. Thus $\operatorname{Mack}_{R}^{\mathcal{X}, \mathcal{Y}}(\mathcal{D})$ is a highest weight category by Theorem 7.2 , or equivalently $\mu_{R}^{\mathcal{X}, \mathcal{Y}}(\mathcal{D})$ is a quasi-hereditary algebra. We exploit BGG reciprocity [19, Lemma $2.5]$, which states in this context that $\left[P_{K, W}: \Delta_{J, U}\right]=\left[\nabla_{J, U}: S_{K, W}\right]$. 
Thus

$$
\begin{aligned}
c_{(H, V),(K, W)} & =\sum_{(J, U)}\left[P_{K, W}: \Delta_{J, U}\right]\left[\Delta_{J, U}, S_{H, V}\right] \\
& =\sum_{(J, U)}\left[\nabla_{J, U}: S_{K, W}\right]\left[\Delta_{J, U}, S_{H, V}\right]
\end{aligned}
$$

over a large enough field. We now compute the numbers $\left[\nabla_{J, U}: S_{K, W}\right]$.

Lemma 8.1. (1) $\left[\nabla_{H, V}^{\mathcal{Y}}: S_{K, W}^{\mathcal{X}, \mathcal{Y}}\right]=\left[\Delta_{H, V^{*}}^{\mathcal{Y}}: S_{K, W^{*}}^{\mathcal{Y}, \mathcal{X}}\right]$ where the superscripts indicate that the functors on the left belong to $\operatorname{Mack}_{R}^{\mathcal{X}, \mathcal{Y}}(\mathcal{D})$ and the functors on the right belong to $\operatorname{Mack}_{R}^{\mathcal{Y}, \mathcal{X}}(\mathcal{D})$.

(2) If $\mathcal{X}=\mathcal{Y}$ and $R$ is a field of characteristic zero we have

$$
\left[\Delta_{H, V^{*}}: S_{K, W^{*}}\right]=\left[\Delta_{H, V}: S_{K, W}\right]
$$

and

$$
\left[\nabla_{H, V}: S_{K, W}\right]=\left[\Delta_{H, V}: S_{K, W}\right]
$$

Proof. (1) This is an application of the duality $*: \operatorname{Mack}_{R}^{\mathcal{X}, \mathcal{Y}} \rightarrow \operatorname{Mack}_{R}^{\mathcal{Y}, \mathcal{X}}$ under which $S_{K, W}^{\mathcal{X}, \mathcal{Y}} \rightarrow S_{K, W^{*}}^{\mathcal{Y}, \mathcal{X}}, \Delta_{H, V}^{\mathcal{X}} \rightarrow \nabla_{H, V^{*}}^{\mathcal{X}}$ and $\nabla_{H, V}^{\mathcal{Y}} \rightarrow \Delta_{H, V^{*}}^{\mathcal{Y}}$, preserving multiplicities in filtrations.

(2) When $\mathcal{X}=\mathcal{Y}$ and $R$ is a field of characteristic zero we have for each group $G$, from Proposition 5.1, that $\Delta_{H, V^{*}}(G) \cong \Delta_{H, V}(G)^{*}$ and $\Delta_{H, V}(G) \cong \nabla_{H, V}(G)$ as $R$ Out $G$-modules, since a module and its dual have isomorphic fixed points. Also in this case $S_{K, W^{*}}(G) \cong$ $\left(S_{K, W}\right)^{*}(G) \cong\left(S_{K, W}(G)\right)^{*}$. Since the multiplicity of a simple functor as a composition factor in another functor $M$ is determined by the modules $M(G)$ as $G$ varies (using the method of [32] and [31]), we see that the composition factors of $\Delta_{H, V^{*}}$ are the duals of the composition factors of $\Delta_{H, V}$, and these are the same as the composition factors of $\nabla_{H, V}$.

Corollary 8.2. Suppose that $\mathcal{X}=\mathcal{Y}$ and let $R$ be an algebraically closed field of characteristic zero. The Cartan matrix of globally defined Mackey functors $\operatorname{Mack}_{R}^{\mathcal{X}, \mathcal{Y}}$ is symmetric.

In fact, we see that to guarantee the symmetry of Cartan invariants such as

$$
c_{(H, V),(K, W)}=c_{(K, W),(H, V)}
$$

we need only suppose that $R$ is a splitting field for all groups Out $L$ where $L$ ranges through the sections of $H$ and $K$. 
Proof. By the last lemma and the preceding remarks we have

$$
\begin{aligned}
c_{(H, V),(K, W)} & =\sum_{(J, U)}\left[\nabla_{J, U}: S_{K, W}\right]\left[\Delta_{J, U}, S_{H, V}\right] \\
& =\sum_{(J, U)}\left[\Delta_{J, U}: S_{K, W}\right]\left[\Delta_{J, U}, S_{H, V}\right]
\end{aligned}
$$

which is symmetric in $(K, W)$ and $(H, V)$.

Our next step in proving that the Cartan matrix of globally defined Mackey functor is symmetric over an arbitrary sufficiently field $R$ when $\mathcal{X}=\mathcal{Y}$ is to invoke a generalization of Brauer's theory of the decomposition map from characteristic zero to characteristic $p$ due to Geck and Rouquier [22, Sect. 2.3]. For the convenience of the reader we give a discussion of this generalization adapted to our purposes here. Let (O) be a complete discrete valuation ring with quotient field $K$ of characteristic 0 and residue field $k$ of characteristic $p$. Let $A_{0}$ be an $\mathbb{O}$-order in a finite dimensional $K$-algebra $A$. We will write $C_{K}$ for the Cartan matrix of $A$-modules and $C_{k}$ for the Cartan matrix of $k \otimes_{\mathbb{O}} A_{0}$-modules. We refer to [5] for standard facts about decomposition modulo $p$.

Every finite dimensional $A$-module $M$ contains a full $A_{0}$-lattice $M_{0}$ (see $[5,1.9 .1])$ and now the theorem of Brauer and Nesbitt states that the composition factors of $k \otimes_{\mathbb{O}} M_{0}$ as a $k \otimes_{\mathbb{O}} A_{0}$-module are determined independently of the choice of $M_{0}$ in $M$. The assignment $[M] \mapsto\left[k \otimes_{\mathbb{O}}\right.$ $\left.M_{0}\right]$ is the decomposition map $d$ in the following commutative square:

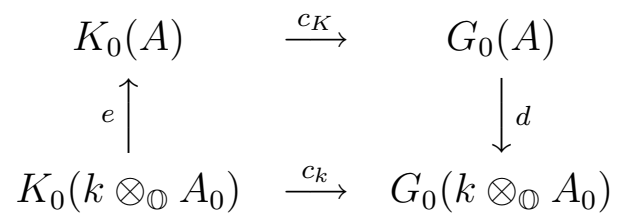

Here we write $K_{0}$ for the Grothendieck group of finitely generated projective modules, and $G_{0}$ for the Grothendieck group of all finitely generated modules, the maps $c_{K}$ and $c_{k}$ being the Cartan homomorphisms. The map $e$ is specified by $[P] \mapsto\left[K \otimes_{\mathbb{O}} \hat{P}\right]$ where $P$ is a projective $k \otimes_{\mathbb{O}} A_{0}$-module and $\hat{P}$ is a projective $A_{0}$-module for which $k \otimes_{\mathbb{O}} \hat{P} \cong P$.

We have bilinear forms

$$
K_{0}(A) \times G_{0}(A) \rightarrow \mathbb{R} \quad \text { and } \quad K_{0}\left(k \otimes_{\mathbb{O}} A_{0}\right) \times G_{0}\left(k \otimes_{\mathbb{O}} A_{0}\right) \rightarrow \mathbb{R}
$$

given by $\langle[P],[M]\rangle=\operatorname{dim}_{K} \operatorname{Hom}(P, M)$ in the first case and $\langle[P],[M]\rangle=$ $\operatorname{dim}_{k} \operatorname{Hom}(P, M)$ in the second, and with respect to these bilinear forms, in both cases, the classes of indecomposable projective modules $\left[P_{S}\right]$ and the classes of simple modules $[S]$ are dual bases so that the forms are non-degenerate. 
Theorem 8.3. (Geck and Rouquier [22, Sect. 2.3]) Let $K$ be a splitting field for $A$ and $k$ a splitting field for $k \otimes_{\mathbb{O}} A_{0}$. With respect to these bilinear forms, $e$ is the transpose of $d$. Thus if $D$ is the matrix of $e$ with respect to the bases of projective modules, then $D^{T}$ is the matrix of $d$ with respect to the bases of simple modules, and the Cartan matrix of $k \otimes_{\mathbb{O}} A_{0}$ is given by

$$
C_{k}=D^{T} C_{K} D
$$

Proof. The proof is the same as that given in $[5,1.9 .6]$ with the final statement modified to account for the fact that $A$ need not be semisimple. Let us write the decomposition matrix as $D=\left(d_{S T}\right)$ where $S$ ranges over simple $A$-modules, $T$ ranges over simple $k \otimes_{\mathbb{O}} A_{0}$-modules, so that $D$ is the transpose of the matrix of $d$. Then

$$
\begin{aligned}
& d_{S T}=\operatorname{dim} \operatorname{Hom}_{k \otimes_{\mathbb{D}} A_{0}}\left(P_{T}, k \otimes_{\mathbb{O}} S_{0}\right) \\
& =\operatorname{rank}_{\mathbb{O}} \operatorname{Hom}_{A_{0}}\left(\hat{P}_{T}, S_{0}\right) \quad \text { since } \hat{P}_{T} \text { is projective } \\
& =\operatorname{dim}_{K} \operatorname{Hom}_{A}\left(K \otimes_{\mathbb{O}} \hat{P}_{T}, S\right) \\
& =\text { multiplicity of } P_{S} \text { as a summand of } K \otimes_{\mathbb{O}} \hat{P}_{T} \text {. }
\end{aligned}
$$

The last number is the $(S, T)$ entry of the matrix of $e$.

Corollary 8.4. In the above situation, if the Cartan matrix $C_{K}$ of $A$ is symmetric, then so is the Cartan matrix $C_{k}$ of $k \otimes_{\mathbb{O}} A_{0}$.

Putting these facts together we obtain one of our main results.

Theorem 8.5. Let $R$ be an algebraically closed field, suppose that $\mathcal{X}=$ $\mathcal{Y}$ and let $\mathcal{D}$ be a section-closed set of finite groups. Then the Cartan matrix of globally defined Mackey functors defined on $\mathcal{D}$ is symmetric.

We now turn to proving that the Cartan matrix of globally defined Mackey functors is non-singular. We first say what we mean by this, bearing in mind that the matrix has infinitely many rows and columns, and in each row or column infinitely many of the entries may be nonzero. We can regard a column as determining an element of the product of copies of $R$ indexed by the simple globally defined Mackey functors. By saying that the Cartan matrix is non-singular we will mean that the columns are linearly independent elements of this space.

Theorem 8.6. Let $R$ be a field, let $\mathcal{D}$ be a section-closed set of finite groups, and in case $R$ has positive characteristic suppose that $\mathcal{X}=\mathcal{Y}$. Then the Cartan matrix of the category $\operatorname{Mack}_{R}^{\mathcal{X}, \mathcal{Y}}(\mathcal{D})$ of globally defined Mackey functors defined on $\mathcal{D}$ is non-singular. 
Proof. Step 1. We claim that it is sufficient to show that the result is true for all finite such sets of groups $\mathcal{D}$. For, if $[P]$ denotes the column of the Cartan matrix corresponding to an indecomposable projective $P$ and we have a linear combination $\sum_{i=1}^{n} \lambda_{i}\left[P_{i}\right]=0$, then there is a finite set of groups $\mathcal{D}$ closed under sections such that all the $P_{i}$ are generated by their values in $\mathcal{D}$. Now by Proposition 3.3 the $P_{i} \downarrow_{\mathcal{D}}$ are indecomposable projectives defined on $\mathcal{D}$ corresponding to distinct columns of the Cartan matrix of $\mathcal{D}$, and $\sum_{i=1}^{n} \lambda_{i}\left[P_{i} \downarrow_{\mathcal{D}}\right]=0$. Assuming that the Cartan matrix for globally defined Mackey functors defined on $\mathcal{D}$ is non-singular, we deduce that $\lambda_{i}=0$ for all $i$.

Step 2. We show that we may assume $R$ is a splitting field. To establish this, let $R \subset R_{1}$ be any separable field extension and consider the corresponding homomorphism of Grothendieck groups

$$
G_{0}\left(\operatorname{Mack}_{R}^{\mathcal{X}, \mathcal{Y}}(\mathcal{D})\right) \rightarrow G_{0}\left(\operatorname{Mack}_{R_{1}}^{\mathcal{X}, \mathcal{Y}}(\mathcal{D})\right) .
$$

Note that if $S$ is any simple globally defined Mackey functor defined over $R$ then the class $\left[R_{1} \otimes_{R} S\right]$ is a sum of simple classes which are disjoint from the simple classes which arise from the other non-isomorphic simples $T$, by considering the effect of an idempotent $e_{S} \in \mu_{R}^{\mathcal{X}, \mathcal{Y}}(\mathcal{D})$ for which $e_{S} S=S$ and $e_{S} T=0$. The effect on indecomposable projectives $P_{S}$ is therefore similar, and $\left[R_{1} \otimes_{R} P_{S}\right]$ is a sum of classes of projective covers of composition factors of $R_{1} \otimes S$ and therefore disjoint from the indecomposable projectives which arise starting from a non-isomorphic $P_{T}$. Assuming that the classes of indecomposable projectives are independent in $G_{0}\left(\operatorname{Mack}_{R_{1}}^{\mathcal{X}, \mathcal{Y}^{\prime}}(\mathcal{D})\right)$ it follows that the $\left[R_{1} \otimes_{R} P_{S}\right]$ are independent there, and hence the $\left[P_{S}\right]$ are independent in $G_{0}\left(\operatorname{Mack}_{R}^{\mathcal{X}, \mathcal{Y}}(\mathcal{D})\right)$ since their images are independent.

Step 3. We suppose that $\mathcal{D}$ is finite and that $K$ is a large enough field of characteristic zero. It was explained before Lemma 8.1 that by BGG reciprocity the Cartan matrix has the form $C_{K}=\Gamma \Theta$ where $\Theta$ is the matrix with entries $\left[\Delta_{J, U}: S_{H, V}\right]$ and $\Gamma$ is the matrix with entries $\left[P_{H, V}: \Delta_{J, U}\right]$. Since both $\Gamma$ and $\Theta$ are unitrianglar it follows that $C_{K}$ is non-singular in this case, regardless of $\mathcal{X}$ and $\mathcal{Y}$. If we assume further that $\mathcal{X}=\mathcal{Y}$ then by Lemma 8.1 and BGG reciprocity we have $\Gamma=\Theta^{T}$, and this decomposition will be used in the rest of the proof for positive characteristic.

Step 4. We assume that $R$ is the field $k$ in a splitting $p$-modular system $(K, \mathbb{O}, k)$. By Theorem 8.3 the Cartan matrix has the form $C_{k}=D^{T} C_{K} D$ where $D$ is the decomposition matrix. Thus on the assumption that $\mathcal{X}=\mathcal{Y}$ we have $C_{k}=(\Theta D)^{T} \Theta D$. We show that $\Theta D$ has maximal rank, by which we mean rank equal to the number of simple globally defined Mackey functors on $\mathcal{D}$ defined over $k$. From this 
it will follow that $C_{k}$ is invertible, as in [31, Lemma 7.4]. (Note that an important hypothesis was omitted from the statement of [31, Lemma 7.4], namely the hypothesis that the matrices under consideration be real. The proof given there is valid with this assumption.) Since $\Theta$ is unitriangular, it suffices to show that $D$ has maximal rank. However this follows by exactly the same argument as that used to prove [31, Cor. 7.5]: the simple globally defined Mackey functors are determined by their list of values at groups $H$ as $k \operatorname{Out}(H)$-modules, and for every group $H$ the decomposition map $G_{0}(K \operatorname{Out}(H)) \rightarrow G_{0}(k \operatorname{Out}(H))$ is known to be surjective by standard theory.

This completes the proof.

\section{SEMisimplicity}

The main result in this section is Theorem 9.5, which says that when $R$ is a field of characteristic zero, $\operatorname{Mack}_{R}^{\mathcal{X}, \mathcal{Y}}$ is semisimple if and only if $\mathcal{X}=\mathcal{Y}=1$. After that we will discuss the semisimplicity of the rings $A_{R}^{\mathcal{X}, \mathcal{Y}}(G, G)$. Since we will allow $\mathcal{X}$ and $\mathcal{Y}$ to vary in this section we will write the functors $S_{H, V}, \Delta_{H, V}$ and $\nabla_{H, V}$ as $S_{H, V}^{\mathcal{X}, \mathcal{Y}}, \Delta_{H, V}^{\mathcal{X}}$ and $\nabla_{H, V}^{\mathcal{Y}}$ to remind us of the category $\operatorname{Mack}_{R}^{\mathcal{X}, \mathcal{Y}}$ to which they belong. We start with a more general result.

Proposition 9.1. Let $\mathcal{X}$ and $\mathcal{Y}$ be sets of finite groups closed under taking sections and extensions, and let $R$ be a field of characteristic zero. In $\mathrm{Mack}_{R}^{\mathcal{X}, 1}$ we have that $\nabla_{H, V}^{1}=S_{H, V}^{\mathcal{X}, 1}$ is simple and $\Delta_{H, V}^{\mathcal{X}}=P_{H, V}^{\mathcal{X}, 1}$ is indecomposable projective. In $\operatorname{Mack}_{R}^{1, \mathcal{Y}}$ we have that $\Delta_{H, V}^{1}=S_{H, V}^{1, \mathcal{Y}}$ is simple and $\nabla_{H, V}^{\mathcal{Y}}=I_{H, V}^{1, \mathcal{Y}}$ is indecomposable injective. Thus the values of the $S_{H, V}^{\mathcal{X}, 1}$ and the $S_{H, V}^{1, \mathcal{Y}}$ are independent of $\mathcal{X}$ and $\mathcal{Y}$ in this situation.

Proof. We start with the explicit description of the simple functors when $\mathcal{X}=\mathcal{Y}=1$, given in [32]:

$$
S_{H, V}^{1,1}(G)=\bigoplus_{\substack{\alpha: H \cong L \leq G \\ \text { up to } G-\text { conjugacy }}} \operatorname{tr}_{L}^{N_{G}(L)}\left({ }^{\alpha} V\right)
$$

for each group $G$. Since $R$ is a field of characteristic zero we have

$$
\operatorname{tr}_{L}^{N_{G}(L)}\left({ }^{\alpha} V\right)={ }^{\alpha} V^{N_{G}(L)}={ }^{\alpha} V_{N_{G}(L)},
$$

and since the double cosets Aut $H \backslash \operatorname{Sec}_{1}(G, H) / G$ biject with the conjugacy classes of subgroups of $G$ isomorphic to $H$ we have

$$
\operatorname{dim} S_{H, V}^{1,1}(G)=\operatorname{dim} \Delta_{H, V}^{1}(G)=\operatorname{dim} \nabla_{H, V}^{1}(G) .
$$


Now $\Delta_{H, V}^{1}$ has $S_{H, V}^{1,1}$ as an image and $\nabla_{H, V}^{1}$ has $S_{H, V}^{1,1}$ as a subfunctor, so $S_{H, V}^{1,1} \cong \Delta_{H, V}^{1} \cong \nabla_{H, V}^{1}$ are simple as functors in Mack ${ }_{R}^{1,1}$. It follows $a$ fortiori that $\Delta_{H, V}^{1}$ is simple as a functor in $\operatorname{Mack}_{R}^{1, \mathcal{Y}}$ and $\nabla_{H, V}^{1}$ is simple as a functor in $\operatorname{Mack}_{R}^{\mathcal{X}, 1}$. From BGG reciprocity (explained before 8.1) we have

$$
\left[P_{H, V}^{\mathcal{X}, 1}: \Delta_{J, U}^{\mathcal{X}}\right]=\left[\nabla_{J, U}^{1}: S_{H, V}^{\mathcal{X}, 1}\right]=\delta_{(H, V),(J, U)}
$$

so that $\Delta_{H, V}^{\mathcal{X}}=P_{H, V}^{\mathcal{X}, 1}$ is indecomposable projective, and by the dual argument $\nabla_{H, V}^{\mathcal{Y}}=I_{H, V}^{1, \mathcal{Y}}$ is indecomposable injective.

As a consequence we obtain one of the implications which will appear in Theorem 9.5.

Corollary 9.2. When $R$ is a field of characteristic zero, $\operatorname{Mack}_{R}^{1,1}$ is semisimple.

Proof. We take $\mathcal{X}=\mathcal{Y}=1$ in Proposition 9.1 and deduce in this situation that $S_{H, V}^{1,1}=P_{H, V}^{1,1}=I_{H, V}^{1,1}$ is simple, injective and projective. From this semisimplicity follows.

Here is a more elaborate version of the same ideas:

Corollary 9.3. Let $\mathcal{X}^{\prime} \subseteq \mathcal{X}$ be closed under taking sections and extensions and let $R$ be a field of characteristic zero. The simple functors in $\operatorname{Mack}_{R}^{\mathcal{X}, 1}$ restrict to simple functors in $\operatorname{Mack}_{R}^{\mathcal{X}^{\prime}, 1}$. The Cartan matrix of $\operatorname{Mack}_{R}^{\mathcal{X}, 1}$ is unitriangular. Every functor in $\mathrm{Mack}^{\mathcal{X}, 1}$ of finite composition length has finite injective dimension. Similar statements hold for $\operatorname{Mack}_{R}^{1, \mathcal{X}}$, except that every finite length functor in $\mathrm{Mack}^{1, \mathcal{X}}$ has finite projective dimension.

Proof. Aside from the fact that they are defined on different sets of morphisms, both $S_{H, V}^{\mathcal{X}, 1}$ and $S_{H, V}^{\mathcal{X}^{\prime}, 1}$ are the same as $\nabla_{H, V}^{1}$ by Proposition 9.1. From this it follows that $S_{H, V}^{\mathcal{X}, 1}$ restricts to $S_{H, V}^{\mathcal{X}^{\prime}, 1}$. Since $\Delta_{H, V}^{\mathcal{X}}=P_{H, V}^{\mathcal{X}, 1}$, again by Proposition 9.1, all composition factors $S_{K, W}^{\mathcal{X}, 1}$ of $P_{H, V}^{\mathcal{X}, 1}$ have $H$ a proper section of $K$, except for a single composition factor $S_{H, V}^{\mathcal{X}, 1}$. Thus if we order the simple functors so that $|H|$ is non-decreasing the Cartan matrix of $\operatorname{Mack}_{R}^{\mathcal{X}, 1}$ is unitriangular.

We deduce from this also that $\operatorname{Ext}\left(S_{K, W}^{\mathcal{X}, 1}, S_{H, V}^{\mathcal{X}, 1}\right)=0$ unless $K$ is a proper section of $H$, and from this it follows that the composition factors of the injective envelope $I_{H, V}^{\mathcal{X}, 1}$ of $S_{H, V}^{\mathcal{X}, 1}$ are all of the form $S_{K, W}^{\mathcal{X}, 1}$ with $K$ a proper section of $H$, apart from a single composition factor $S_{H, V}^{\mathcal{X}, 1}$. This shows that the matrix of composition factor multiplicities of 
the indecomposable injectives is unitriangular, and from this it follows by duality that the Cartan matrix of $\operatorname{Mack}_{R}^{1, \mathcal{X}}$ is unitriangular.

It follows from this also that each finite length functor in $\operatorname{Mack}_{R}^{1, \mathcal{X}}$ has a finite injective resolution. Given such a functor $M$ we may find a monomorphism to an injective functor so that the cokernel has composition factors associated to groups which are all proper sections of at least one of the groups associated to composition factors of $M$. Repeating this process, the groups get strictly smaller at each stage and it must eventually terminate. The fact that finite length functors in $\mathrm{Mack}^{1, \mathcal{X}}$ have finite projective dimension follows by duality.

We relate the different functors $\Delta$ and $S$ which arise as $\mathcal{X}$ and $\mathcal{Y}$ vary, by way of preparation for Theorem 9.5.

Proposition 9.4. Let $\mathcal{X}, \mathcal{Y}, \mathcal{X}^{\prime}, \mathcal{Y}^{\prime}$ be sets of finite groups closed under taking sections and extensions. If $K$ is a group lying in both $\mathcal{X}$ and $\mathcal{X}^{\prime}$ then $S_{H, V}^{\mathcal{X}, \mathcal{Y}}(K)=S_{H, V}^{\mathcal{X}^{\prime}, \mathcal{Y}}(K)$ and $\Delta_{H, V}^{\mathcal{X}}(K)=\Delta_{H, V}^{\mathcal{X}^{\prime}}(K)$ for all $H$ and $V$. Similarly, if $K$ is a group lying in both $\mathcal{Y}$ and $\mathcal{Y}^{\prime}$ then $S_{H, V}^{\mathcal{X}, \mathcal{Y}}(K)=$ $S_{H, V}^{\mathcal{X}, \mathcal{Y}^{\prime}}(K)$ and $\nabla_{H, V}^{\mathcal{Y}}(K)=\nabla_{H, V}^{\mathcal{Y}^{\prime}}(K)$ for all $H$ and $V$.

Proof. Suppose $K$ lies in both $\mathcal{X}$ and $\mathcal{X}^{\prime}$ and let $\mathcal{D}$ be the full subcategory of $\mathcal{C}_{R}^{\mathcal{X}, \mathcal{Y}}$ whose objects are the sections of $K$. Now by Proposition $3.1 S_{H, V}^{\mathcal{X}, \mathcal{Y}} \downarrow_{\mathcal{D}}$ and $S_{H, V}^{\mathcal{X}^{\prime}, \mathcal{Y}} \downarrow_{\mathcal{D}}$ are the simple functors in $\operatorname{Mack}_{R}^{\mathcal{X}, \mathcal{Y}}(\mathcal{D})$ and $\operatorname{Mack}_{R}^{\mathcal{X}^{\prime}, \mathcal{Y}}(\mathcal{D})$ parametrized by $(H, V)$. However $\operatorname{Mack}_{R}^{\mathcal{X}, \mathcal{Y}}(\mathcal{D})=$ $\operatorname{Mack}_{R}^{\mathcal{X}^{\prime}, \mathcal{Y}}(\mathcal{D})$ since the groups whose isomorphism types lie in both $\mathcal{X}$ and $\mathcal{D}$ are the same as those lying in both $\mathcal{X}^{\prime}$ and $\mathcal{D}$. Thus $S_{H, V}^{\mathcal{X}, \mathcal{Y}}(K)=$ $S_{H, V}^{\mathcal{X}^{\prime}, \mathcal{Y}}(K)$. A similar argument proves the corresponding assertion for $\Delta$ using Corollary 5.4 .

We deduce the second half of the statement of the proposition from the first using duality.

Theorem 9.5. Let $\mathcal{X}$ and $\mathcal{Y}$ be sets of finite groups closed under taking sections and extensions, let all be the class of all finite groups, and let $R$ be a field of characteristic zero. Then $\operatorname{Mack}_{R}^{\mathcal{X}, \mathcal{Y}}$ (all) is semisimple if and only if $\mathcal{X}=\mathcal{Y}=1$.

Proof. We show that if either $\mathcal{X} \neq 1$ or $\mathcal{Y} \neq 1$ then the category of functors $\operatorname{Mack}_{R}^{\mathcal{X}, \mathcal{Y}}$ is not semisimple, the other half of the proof having been dealt with in Proposition 9.2. Since $\operatorname{Mack}_{R}^{\mathcal{X}, \mathcal{Y}}$ is in duality with $\operatorname{Mack}_{R}^{\mathcal{Y}, \mathcal{X}}$, each of these categories is semisimple if the other one is, and so we will only treat the case $\mathcal{X} \neq 1$. Our approach will be to show that $\Delta_{1, R}^{\mathcal{X}} \neq S_{1, R}^{\mathcal{X}, \mathcal{Y}}$, and since by Corollary $5.5 \Delta_{1, R}^{\mathcal{X}}$ has $S_{1, R}^{\mathcal{X}, \mathcal{Y}}$ as its unique 
simple quotient it will follow that $\Delta_{1, R}^{\mathcal{X}}$ is not a direct sum of simple functors.

Supposing now that $\mathcal{X} \neq 1$, there is some non-identity group in $\mathcal{X}$, and hence for some prime $p, \mathcal{X}$ contains all $p$-groups since $\mathcal{X}$ is supposed to be closed under taking extensions and sections. From its definition we have that $\Delta_{1, R}^{\text {all }}=b$, the Burnside ring functor, regardless of what $\mathcal{Y}$ is. It is shown in [7] that there is a $p$-group $K$ for which $S_{1, R}^{\text {all,all }}(K) \neq b(K)$ (in the terminology of Bouc, a $b$-group will have this property). Since $K$ lies in $\mathcal{X}$ we have by Proposition 9.4 that

$$
S_{1, R}^{\mathcal{X}, \text { all }}(K)=S_{1, R}^{\text {all,all }}(K) \neq b(K)=\Delta_{1, R}^{\text {all }}(K)=\Delta_{1, R}^{\mathcal{X}}(K) .
$$

In fact the left hand side has smaller dimension than the right since $S_{1, R}^{\text {all,all }}$ is an image of $b$. We claim that $\operatorname{dim} S_{1, R}^{\mathcal{X}, \mathcal{Y}}(K) \leq \operatorname{dim} S_{1, R}^{\mathcal{X} \text {,all }}(K)$. This is because we may restrict the category of definition of $S_{1, R}^{\mathcal{X} \text {,all }}$ from $\mathcal{C}_{R}^{\mathcal{X} \text {,all }}$ to $\mathcal{C}_{R}^{\mathcal{X}, \mathcal{Y}}$, and now it is a functor which must have $S_{1, R}^{\mathcal{X}, \mathcal{Y}}$ as a composition factor since the latter is the only simple functor which does not vanish at 1 . Putting all this together we have $\operatorname{dim} S_{1, R}^{\mathcal{X}, \mathcal{Y}}(K) \leq$ $\operatorname{dim} S_{1, R}^{\mathcal{X}, \text { all }}(K)<\operatorname{dim} \Delta_{1, R}^{\mathcal{X}}(K)$, and we conclude that $S_{1, R}^{\mathcal{X}, \mathcal{Y}} \neq \Delta_{1, R}^{\mathcal{X}}$. As commented earlier this implies that $\operatorname{Mack}_{R}^{\mathcal{X}, \mathcal{Y}}$ is not semisimple.

It is a very interesting question to study the structure of the rings $A_{R}^{\mathcal{X}, \mathcal{Y}}(G, G)$ which arise as the endomorphism rings of the objects in $\mathcal{C}_{R}^{\mathcal{X}, \mathcal{Y}}$, and also as the endomorphism rings of the representable globally defined Mackey functors $A_{R}^{\mathcal{X}, \mathcal{Y}}(, G)$. It is a theorem of Bouc ([7] and private communication) and Barker [4] that when $R$ is a field of characteristic zero the $\operatorname{ring} A_{R}^{\text {all,all }}(G, G)$ is semisimple if and only if $G$ is cyclic. We conclude this section with a result about the semisimplicity of such rings for different $\mathcal{X}$ and $\mathcal{Y}$.

Theorem 9.6. (1) Let $R$ be a field of characteristic zero and $G$ a finite group. The ring $A_{R}^{1,1}(G, G)$ is semisimple.

(2) The ring $A_{R}^{\text {all,1 }}\left(C_{2}, C_{2}\right)$ is not semisimple for any choice of ring $R$.

(3) The representable functor $A_{R}^{\text {all,1 }}\left(, C_{2}\right)$ is not a direct sum of simple functors for any choice of ring $R$.

Proof. (1) This follows from Corollary 9.2 (half of Theorem 9.5) since the representable functor $A_{R}^{1,1}(, G)$ is semisimple and by Yoneda's lemma has $A_{R}^{1,1}(G, G)$ as its endomorphism ring.

(2) We compute the structure of $A_{R}^{\text {all,1 }}\left(C_{2}, C_{2}\right)$ in the most direct way. There are, up to isomorphism, three transitive $C_{2} \times C_{2}$-bisets with identity stabilizers in the second factor. These are: the identity 
element in the ring $A_{R}^{\text {all,1}}\left(C_{2}, C_{2}\right)$, which is the set $C_{2}$ with both $C_{2}$ factors acting regularly, and which we denote 1 ; another set $C_{2}$ with the second $C_{2}$ factor acting regularly and the first factor acting trivially, which we denote $A$; and lastly the regular representation $R$ of $C_{2} \times C_{2}$. These bisets form a basis of $A_{R}^{\text {all, } 1}\left(C_{2}, C_{2}\right)$. We readily compute that $A \circ R=2 A$ and $R \circ A=R$, so $A_{\mathcal{X}, \mathcal{Y}}^{R}\left(C_{2}, C_{2}\right)$ is non-commutative. Since this ring has dimension 3 it cannot be semisimple, for otherwise it would have at least a $2 \times 2$ matrix summand (over an algebraically closed extension field).

(3) By Yoneda's lemma, the representable functor $A_{R}^{\text {all,1 }}\left(, C_{2}\right)$ has $A_{R}^{\text {all, }}\left(C_{2}, C_{2}\right)$ as its endomorphism ring, and if it were a direct sum of simple functors its endomorphism ring would be semisimple.

\section{Classifying spaces of Finite groups}

We describe an application to stable homotopy classes of maps

$$
\left[\left(B G_{+}\right)_{p}^{\wedge},\left(B K_{+}\right)_{p}^{\wedge}\right]
$$

where $B G_{+}$denotes the suspension spectrum constructed from the classifying space $B G$ to which a base point has been adjoined, and $(-)_{p}^{\wedge}$ denotes $p$-completion. We will see that global Mackey algebras arise naturally in this context.

Proposition 10.1. Let $\mathcal{D}$ be a finite, section-closed set of finite groups and consider the $p$-completed suspension spectrum $B=\bigvee_{G \in \mathcal{D}}\left(B G_{+}\right)_{p}^{\wedge}$. Then the endomorphism ring of stable maps $[B, B]$ is Morita equivalent to the global Mackey algebra $\mu_{\mathbb{Z}_{p}}^{1, \text { all }}\left(\mathcal{D}_{p}\right)$, where $\mathcal{D}_{p}$ consists of the $p$ groups in $\mathcal{D}$

Proof. As described in [32], there is an equivalence of categories between the full subcategory of the category of spectra whose objects are the wedges of summands of the $\left(B G_{+}\right)_{p}^{\wedge}$, and the full subcategory of Mack $\mathbb{Z}_{\mathbb{Z}_{p}}^{\text {all,1 }}$ whose objects are the direct sums of indecomposable projectives $P_{H, V}$ with $H$ a $p$-group. The indecomposable summands of $\left(B G_{+}\right)_{p}^{\wedge}$ all correspond under this equivalence to projectives of the form $P_{H, V}$ with $H$ a $p$-subgroup of $G$, and every $P_{H, V}$ for which $H$ is a Sylow $p$-subgroup of $G$ does arise in this way. It follows that the

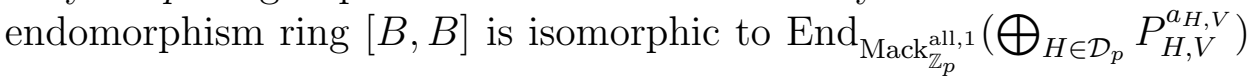
where the $a_{H, V}$ are certain multiplicities, each projective $P_{H, V}$ occuring with non-zero multiplicity. Since these are exactly the indecomposable projectives in $\operatorname{Mack}_{\mathbb{Z}_{p}}^{\text {all,1}}\left(\mathcal{D}_{p}\right)$ (see [32]), which is the same as the category of $\mu_{\mathbb{Z}_{p}}^{\text {all,1}}\left(\mathcal{D}_{p}\right)$-modules, $[B, B]$ is Morita equivalent to the opposite 
$\operatorname{ring}\left(\mu_{\mathbb{Z}_{p}}^{\text {all,1}}\left(\mathcal{D}_{p}\right)\right)^{\text {op }}$. This in turn is isomorphic to $\mu_{\mathbb{Z}_{p}}^{1, \text { all }}\left(\mathcal{D}_{p}\right)$ via the antiisomorphism which sends a biset to its opposite.

The following is our main application to the stable maps between spectra, giving a statement which is outside the context of Mackey functors.

Corollary 10.2. Let $B$ be the spectrum $B=\bigvee_{G \in \mathcal{D}}\left(B G_{+}\right)_{p}^{\wedge}$ as above. Then, on extending scalars to $\mathbb{Q}_{p}$, the endomorphism ring of stable maps $[B, B] \otimes_{\mathbb{Z}_{p}} \mathbb{Q}_{p}$ is quasi-hereditary.

Proof. This is immediate from Proposition 10.1 and Theorem 7.2.

In Section 11.4 we will calculate the Cartan matrix of $[B, B] \otimes_{\mathbb{Z}_{p}} \mathbb{Q}_{p}$ and also of $[B, B] \otimes_{\mathbb{Z}_{p}} \mathbb{F}_{p}$ in the case where $p=2$ and $\mathcal{D}_{p}$ consists of the sections of the cyclic, dihedral and quaternion groups of order 8 .

\section{Calculation of Cartan and decomposition matrices}

11.1. Overview of the calculations. The novel feature we have introduced in this paper is that highest weight theory provides a method for computing the Cartan matrix of $\operatorname{Mack}_{R}^{\mathcal{X}, \mathcal{Y}}$ when $R$ is a field of characteristic zero, using formula 8.1 and Corollary 8.2 which states that the Cartan matrix is symmetric. We then compute Cartan matrices in positive characteristic by applying Theorem 8.3 which gives a formula in terms of the decomposition matrix and the Cartan matrix in characteristic zero.

In these calculations we take $\mathcal{D}$ to be the set of sections of $C_{8}, D_{8}$ and $Q_{8}$, the cyclic, dihedral and quaternion groups of order 8 . For these groups $R=\mathbb{Q}$ and $R=\mathbb{F}_{2}$ are splitting fields for all groups Out $H$ which arise. The Cartan matrices we compute are thus the Cartan matrices of the algebras $\mu_{R}^{\mathcal{X}, \mathcal{Y}}(\mathcal{D})$ for this choice of $\mathcal{D}$, and by Proposition 3.3 they are also the parts of the Cartan matrices for $\mu_{R}^{\mathcal{X}, \mathcal{Y}}$ (all) whose rows and columns are labelled by pairs $(H, V)$ where $H \in \mathcal{D}$.

To compute decomposition matrices we use the method introduced in [31] and continued in [32]. To each globally defined Mackey functor $M$ defined on $\mathcal{D}$ we associate the list of evaluations $([M(G)])_{G \in \mathcal{D}}$ where $[M(G)]$ denotes the element of the Grothendieck group $G_{0}(R$ Out $G)$ determined by $M(G)$. This list of elements of Grothendieck groups we call the formal character of $M$, a term introducted in this context in [35]. As explained in [35], we may regard the evaluations $M(G)$ as the weight spaces of $M$, by analogy with Lie theory. The formal character of $M$ determines its composition factors, as observed in [32]. 
We present the formal character of a functor as a column in a table where the rows are indexed by pairs $(K, W)$ where $K$ ranges over all finite groups and $W$ ranges over simple $R$ Out $K$-modules. The corresponding entry in the table is the multiplicity with which $[W]$ appears in $[M(K)]$. In the case where the functors $M$ are the simple functors $S_{H, V}$ this table of values $\left[S_{H, V}(G)\right]$ was denoted $\Psi$ in [31] and [32].

The matrices $\Psi$ of formal characters of simple functors are used in situations where composition factor multiplicities need to be computed. This arises in the computation of decomposition matrices, since for these we reduce simple functors from characteristic 0 to characteristic $p$ and ask for the composition factor multiplicities of the result. In this situation the formal character of the reduction $\bmod p$ of a functor is obtained by reducing $\bmod p$ each of the terms in the characteristic zero formal character. We obtain the composition factors of the reduction by expressing the reduced formal character as a linear combination of the formal characters of the simple functors in characteristic $p$. The matrices $\Psi$ are also used in computing Cartan matrices using formula 8.1. Here we need to know the composition factors of $\Delta$ functors, and these are obtained by expressing the formal character of the $\Delta$ as a linear combination of the formal characters of the simple functors.

11.2. Detail of the calculations. These paragraphs are technical and can be skipped unless the reader has a particular interest in the calculations.

In calculating the matrices of formal characters of the $\Delta$ and $\nabla$ functors we compute directly the expressions given in Proposition 5.1. The computation of matrices $\Psi$ of formal characters of the simple functors then proceeds from this using Corollary 5.6 and the remarks at the start of Section 4 and Proposition 4.1, since $S_{H, V}$ is the quotient of $\Delta_{H, V}$ cogenerated by its value at $H$. Thus, according to Proposition $4.1(3)$, to obtain $S_{H, V}(G)$ we factor out from $\Delta_{H, V}(G)$ the value of the subfunctor

$$
R_{\{H\}} \Delta_{H, V}(G)=\bigcap_{\Omega: G \rightarrow H} \operatorname{Ker} \Delta_{H, V}(\Omega)
$$

where $\Omega$ ranges over $(H, G)$-bisets with $G$-stabilizers in $\mathcal{Y}$, and because $\Delta_{H, V}$ is zero on groups which do not have $H$ as a section, we only need consider those $\Omega$ for which the $H$-stabilizer is 1 (i.e. which do not factor through a proper section of $H$ ). Such $\Omega$ are determined by a section of $G$ isomorphic to $H$, or in other words a surjection $\alpha: A \rightarrow H$ where $A \leq G$. Writing $B=\operatorname{Ker} \alpha \in \mathcal{Y}$ (so that $H \cong A / B$ ) there is a corresponding biset $\Omega_{\alpha}={ }_{A / B}(B \backslash G)_{G}={ }_{H} H_{A} \circ{ }_{A} G_{G}$. For each 
such section, taken up to conjugacy in $G$ and up to automorphisms of $H$, we form the corresponding linear map $\Omega_{\alpha}: \Delta_{H, V}(G) \rightarrow \Delta_{H, V}(H)$. Factoring out from $\Delta_{H, V}(G)$ the common kernel of all these linear maps gives $S_{H, V}(G)$.

In fact, $\Delta_{H, V}(G)$ itself may be constructed by means of the opposites of the bisets just considered, but with $\operatorname{Ker} \alpha \in \mathcal{X}$. Since $\Delta_{H, V}$ is generated by its value at $H$ and is zero on groups which do not have $H$ as a section, $\Delta_{H, V}(G)$ is the sum of all images of maps $\Omega_{\alpha}^{\mathrm{op}}: \Delta_{H, V}(H) \rightarrow$ $\Delta_{H, V}(G)$. According to the formula of Proposition 5.1 these images lie in different summands of $\Delta_{H, V}(G)$ and so we can recast that formula as

$$
\Delta_{H, V}(G) \cong \underset{\alpha \in\left[\text { Aut } H \backslash \operatorname{Sec}_{\mathcal{X}}(G, H) / G\right]}{ } \Omega_{\alpha}^{\mathrm{op}}(V) .
$$

Computing $R_{\{H\}} \Delta_{H, V}(G)$ is now a question of computing the kernel of the matrix $\left(\Omega_{\beta} \Omega_{\alpha}^{\mathrm{op}}\right)_{\alpha, \beta}$ where

$$
\alpha \in\left[\operatorname{Aut} H \backslash \operatorname{Sec}_{\mathcal{X}}(G, H) / G\right], \quad \beta \in\left[\operatorname{Aut} H \backslash \operatorname{Sec}_{\mathcal{Y}}(G, H) / G\right]
$$

and each entry is taken to be the endomorphism of the vector space $V$ determined by the biset indicated. Thus $S_{H, V}(G)$ identifies as the cokernel of this matrix, regarded as a quotient of $\Delta_{H, V}(G)$. When $\operatorname{dim} V=1$ the endomorphism of $V$ determined by each biset $\Omega_{\beta} \Omega_{\alpha}^{\text {op }}$ is multiplication by a scalar, and $\operatorname{dim} S_{H, V}(G)$ is the rank of the bilinear form whose matrix has these scalar entries.

We illustrate with a small example by computing $S_{C_{2}, R}^{\text {all,all }}\left(Q_{8}\right)$ for all fields $R$. The quaternion group $Q_{8}$ has three subgroups $A, B$ and $C$ which are cyclic of order 4 and a central subgroup $Z$ of order 2 . In the following matrix the rows are indexed by the $\left(C_{2}, Q_{8}\right)$-bisets for which $C_{2}$ with trivial stabilizers, and the columns are indexed by their opposites. With the biset $Z \backslash Q_{8}$ and its opposite $Q_{8} / Z$ there are three possible actions of $C_{2}$, as $A / Z, B / Z$ and $C / Z$, and each of these three

\begin{tabular}{|c|c|c|c|c|c|c|c|}
\hline & $Q_{8} / A$ & $Q_{8} / B$ & $Q_{8} / C$ & $Q_{8} / Z$ & $Q_{8} / Z$ & $Q_{8} / Z$ & $Q_{8}$ \\
\hline$A \backslash Q_{8}$ & 1 & 0 & 0 & 0 & 1 & 1 & 0 \\
\hline$B \backslash Q_{8}$ & 0 & 1 & 0 & 1 & 0 & 1 & 0 \\
\hline$C \backslash Q_{8}$ & 0 & 0 & 1 & 1 & 1 & 0 & 0 \\
\hline$Z \backslash Q_{8}$ & 0 & 1 & 1 & 2 & 0 & 0 & 0 \\
\hline$Z \backslash Q_{8}$ & 1 & 0 & 1 & 0 & 2 & 0 & 0 \\
\hline$Z \backslash Q_{8}$ & 1 & 1 & 0 & 0 & 0 & 2 & 0 \\
\hline$Q_{8}$ & 0 & 0 & 0 & 0 & 0 & 0 & 4 \\
\hline
\end{tabular}
actions is supposed to occur among the bisets shown.

As an example, the entry in row 1 column 4 is computed by taking the biset product $\left(A \backslash Q_{8}\right) \circ\left(Q_{8} / Z\right)$ where the second biset has an action 
of $C_{2}$ via $A / Z$. This product has two elements permuted freely by the group $C_{2}$ on the left, but fixed by the $C_{2}$ on the right. Since the action on one side is not free, the biset factors through 1 and acts as zero on $\Delta_{C_{2}, R}$, giving a matrix entry 0 . On the other hand when the action on the right is via $B / Z$ or $C / Z$ the product biset is two elements with a free action on each side, giving an entry 1 . The rank of this matrix is 7 unless $R$ has characteristic 2 , when the rank is 6 , so we deduce

$$
\operatorname{dim} S_{C_{2}, R}^{\text {all,all }}\left(Q_{8}\right)= \begin{cases}7 & \text { if } \operatorname{char} R \neq 2, \\ 6 & \text { if } \operatorname{char} R=2 .\end{cases}
$$

Furthermore we see that $\operatorname{Out}\left(Q_{8}\right) \cong S_{3}$ permutes the images of the various bisets in $\Delta_{C_{2}, R}\left(Q_{8}\right)$ as two copies of the permutation representation on three points, together with a copy of the trivial representation, and this is the $R$ Out $Q_{8}$-module structure of $S_{C_{2}, R}^{\text {all,all }}\left(Q_{8}\right)$ except that in characteristic 2 we lose a copy of the trivial representation. This information provides an entry in Table 11 and an entry in Table 14.

We give another example with cyclic groups.

Proposition 11.1. Let $R$ be a field and $p$ a prime. Then

$$
\operatorname{dim} S_{C_{p^{r}, R}}^{\text {all,all }}\left(C_{p^{r+s}}\right)= \begin{cases}s+1 & \text { if } \operatorname{char}(R) \neq p \text { or } s=0, \\ 2 & \text { if } r=0, s>0 \text { and } \operatorname{char}(R)=p, \\ 1 & \text { if } r>0 \text { and } \operatorname{char}(R)=p,\end{cases}
$$

Proof. When $r=0$ the matrix with entries $\Omega_{\beta} \Omega_{\alpha}^{\text {op }}$ has the form

$$
\left(\begin{array}{lllll}
1 & 1 & 1 & 1 & \\
1 & p & p & p & \\
1 & p & p^{2} & p^{2} & \\
1 & p & p^{2} & p^{3} & \\
& & & & \ddots
\end{array}\right)
$$

and provided $s>0$ this has rank 2 or $s+1$ or according as $\operatorname{char}(R)$ is or is not $p$. When $r>0$ the matrix is diagonal with entries $1, p, p^{2}, \ldots, p^{s}$ and this has rank 1 or $s+1$ according as $\operatorname{char}(R)$ is or is not $p$. In any case, the matrix has rank 1 when $s=0$.

11.3. Cartan, decomposition and formal character matrices when $\mathcal{X}=\mathcal{Y}=1$. Theorem 9.5 implies that in characteristic 0 the Cartan matrix is the identity matrix when $\mathcal{X}=\mathcal{Y}=1$.

We present the matrices $\Psi$ of formal characters of simple functors. When $R$ is a field of characteristic 0 we have $S_{H, V}^{\mathcal{X}, 1}=\nabla_{H, V}^{1}$ independently of $\mathcal{X}$ by Proposition 9.1, and we compute Table 2 directly from the expression for $\nabla_{H, V}^{1}$ given in Proposition 5.1. Table 2 will be used 
a second time when we consider $\mathcal{X}=$ all and $\mathcal{Y}=1$. Table 3 appears already in [32] and we copy it from that source. From these tables we compute in Tables 4 and 5 the decomposition and Cartan matrices at the prime 2 using Theorem 8.3. 
TABLE 2. Formal characters of simple functors over $\mathbb{Q}$

\begin{tabular}{|c|c|c|c|c|c|c|c|c|}
\hline \multirow[b]{2}{*}{$\Psi$} & \multicolumn{8}{|c|}{$S_{H, V}^{\mathcal{X}, 1}=\nabla_{H, V}^{1}$ over $\mathbb{Q}$} \\
\hline & $\begin{array}{l}1 \\
1\end{array}$ & $\begin{array}{c}C_{2} \\
1\end{array}$ & $\begin{array}{l}C_{4} \\
1-1\end{array}$ & $\begin{array}{l}\left(C_{2}\right)^{2} \\
1-12\end{array}$ & $\begin{array}{ll}C_{8} & \\
1-1_{3}-1_{5}-\end{array}$ & $-1_{7}$ & $\begin{array}{lll} & & \\
Q_{8} & -12\end{array}$ & $\begin{array}{ll}D_{8} & \\
1-1\end{array}$ \\
\hline $\begin{array}{ll}1 & 1\end{array}$ & 1 & & & & & & & \\
\hline $\begin{array}{ll}C_{2} & 1\end{array}$ & 1 & 1 & & & & & & \\
\hline $\begin{array}{lr}C_{4} & 1 \\
& -1\end{array}$ & 1 & 1 & $\begin{array}{ll}1 & \\
& \\
\end{array}$ & & & & & \\
\hline $\begin{array}{lr}\left(C_{2}\right)^{2} & 1 \\
& -1 \\
& 2\end{array}$ & 1 & 1 & & $\begin{array}{lll}1 & & \\
& 1 & \\
& & 1\end{array}$ & & & & \\
\hline $\begin{array}{lr}C_{8} & 1 \\
& -1_{3} \\
& -1_{5} \\
& -1_{7}\end{array}$ & 1 & 1 & 1 & & $\begin{array}{lll}1 & & \\
& 1 & \\
& & 1\end{array}$ & 1 & & \\
\hline $\begin{array}{lr}Q_{8} & 1 \\
& -1 \\
& 2\end{array}$ & 1 & 1 & $\begin{array}{l}1 \\
1\end{array}$ & & & & \begin{tabular}{|lll}
1 & & \\
& 1 & \\
& & 1
\end{tabular} & \\
\hline $\begin{array}{rr}D_{8} & 1 \\
& -1\end{array}$ & 1 & $\begin{array}{l}2 \\
1\end{array}$ & 1 & $\begin{array}{ll}1 & 1 \\
1 & 1\end{array}$ & & & & $\begin{array}{ll}1 & \\
& 1\end{array}$ \\
\hline
\end{tabular}

TABLE 3. Formal characters of simple functors over $\mathbb{F}_{2}$.

\begin{tabular}{|c|c|c|c|c|c|c|c|}
\hline \multirow[b]{2}{*}{$\Psi$} & \multicolumn{7}{|c|}{$S_{H, V}^{1,1}$ over $\mathbb{F}_{2}$} \\
\hline & \begin{tabular}{|l|}
1 \\
1
\end{tabular} & $\begin{array}{c}C_{2} \\
1\end{array}$ & $\begin{array}{c}C_{4} \\
1\end{array}$ & $\begin{array}{c}\left(C_{2}\right)^{2} \\
1\end{array}$ & $\begin{array}{c}C_{8} \\
1\end{array}$ & $\begin{array}{c}Q_{8} \\
12\end{array}$ & $\begin{array}{c}D_{8} \\
1\end{array}$ \\
\hline 11 & 1 & & & & & & \\
\hline$C_{2} 1$ & & 1 & & & & & \\
\hline$C_{4} 1$ & & & 1 & & & & \\
\hline$\left(C_{2}\right)^{2}{ }_{2}^{1}$ & & & & 1 & & & \\
\hline$C_{8} 1$ & & & & & 1 & & \\
\hline $\begin{array}{r}Q_{8} 1 \\
2\end{array}$ & & & & & & $\begin{array}{lll} & 1 & \\
& & 1\end{array}$ & \\
\hline$D_{8} 1$ & & & & 2 & & & 1 \\
\hline
\end{tabular}


TABLE 4. Decomposition matrix from $\operatorname{Mack}_{\mathbb{Q}}^{1,1}$ to $\operatorname{Mack}_{\mathbb{F}_{2}}^{1,1}$

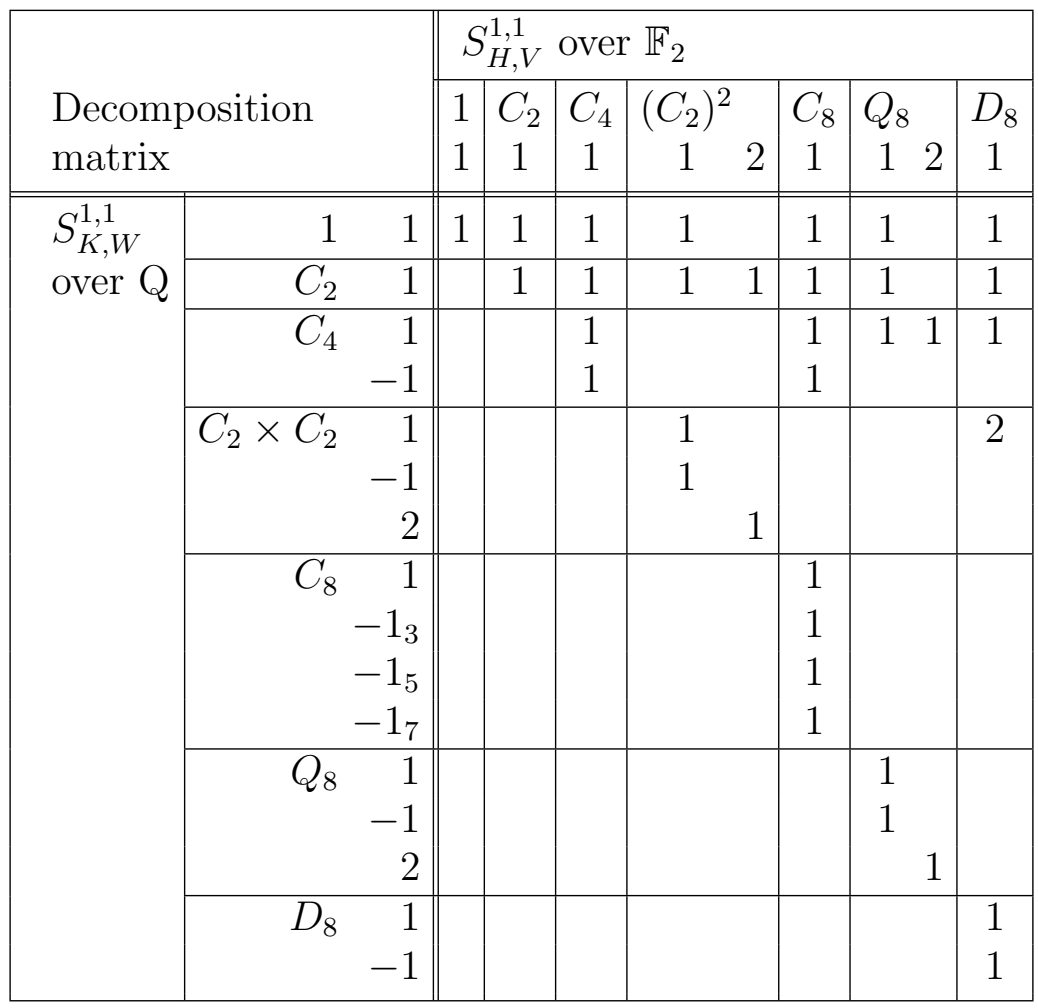

TABLE 5. Cartan matrix of $\operatorname{Mack}_{\mathbb{F}_{2}}^{1,1}$

\begin{tabular}{|c|c|c|c|c|c|c|c|c|c|}
\hline \multirow{2}{*}{\multicolumn{2}{|c|}{ Cartan matrix }} & \multicolumn{8}{|c|}{$P_{H, V}^{1,1}$ over $\mathbb{F}_{2}$} \\
\hline & & \begin{tabular}{l|}
1 \\
1
\end{tabular} & $\begin{array}{c}C_{2} \\
1\end{array}$ & $\begin{array}{c}C_{4} \\
1\end{array}$ & \multicolumn{2}{|c|}{$\left(C_{2}\right)^{2}$} & $\begin{array}{c}C_{8} \\
1\end{array}$ & $\begin{array}{l}Q_{8} \\
12\end{array}$ & $\begin{array}{c}D_{8} \\
1\end{array}$ \\
\hline \multirow{9}{*}{$\begin{array}{l}S_{K, W}^{1,1} \\
\text { over } \mathbb{F}_{2}\end{array}$} & 11 & 1 & 1 & 1 & 1 & 0 & 1 & 10 & 1 \\
\hline & $C_{2} 1$ & 1 & 2 & 2 & 2 & 1 & 2 & 20 & 2 \\
\hline & $C_{4} 1$ & 1 & 2 & 4 & 2 & 1 & 4 & 31 & 3 \\
\hline & $\left(C_{2}\right)^{2} 1$ & 1 & 2 & 2 & 4 & 1 & 2 & 20 & 4 \\
\hline & 2 & 0 & 1 & 1 & 1 & 2 & 1 & 10 & 1 \\
\hline & $C_{8} 1$ & 1 & 2 & 4 & 2 & 1 & 8 & 31 & 3 \\
\hline & $Q_{8} 1$ & 1 & 2 & 3 & 2 & 1 & 3 & 51 & 3 \\
\hline & 2 & 0 & 0 & 1 & 0 & 0 & 1 & 12 & 1 \\
\hline & $D_{8} 1$ & 1 & 2 & 3 & 4 & 1 & 3 & $\begin{array}{ll}31 \\
\end{array}$ & 9 \\
\hline
\end{tabular}


11.4. Cartan, decomposition and formal character matrices when $\mathcal{X}=$ all and $\mathcal{Y}=1$. Observe that for any choice of $\mathcal{X}$ and $\mathcal{Y}$ and field $R$ the duality between $\operatorname{Mack}_{R}^{\mathcal{X}, \mathcal{Y}}$ and $\operatorname{Mack}_{R}^{\mathcal{Y}, \mathcal{X}}$ means that the Cartan matrix of Mack $_{R}^{\mathcal{Y}, \mathcal{X}}$ is equal to the matrix of composition factor multiplicities in the indecomposable injective objects of $\operatorname{Mack}_{R}^{\mathcal{X}, \mathcal{Y}}$. Provided that $R$ is large enough, this is the transpose of the Cartan matrix of $\operatorname{Mack}_{R}^{\mathcal{X}, \mathcal{Y}}$. Thus the Cartan matrices of $\operatorname{Mack}_{R}^{\text {all,1 }}$ which we will compute in this section are the transposes of the Cartan matrices of Mack ${ }_{R}^{1, a l l}$. By the discussion of Section 10 these are the Cartan matrices of the algebras $[B, B] \otimes_{\mathbb{Z}_{p}} \mathbb{Q}_{p}$ and $[B, B] \otimes_{\mathbb{Z}_{p}} \mathbb{F}_{p}$.

We start by computing the Cartan matrix in characteristic zero. Here we know that $\Delta_{H, V}^{\text {all }}=P_{H, V}^{\text {all,1 }}$ by Proposition 9.1, and we present the table of formal characters of these functors which is computed directly from Proposition 5.1.

TABLE 6. Formal characters of projectives $P_{H, V}^{\text {all, }}$ over $\mathbb{Q}$

\begin{tabular}{|c|c|c|c|c|c|c|c|c|c|}
\hline \multirow[b]{2}{*}{$\Psi$} & \multicolumn{9}{|c|}{$P_{H, V}^{\text {all, }}=\Delta_{H, V}^{\text {all }}$ over $\mathbb{Q}$} \\
\hline & $\begin{array}{l}1 \\
1\end{array}$ & $\begin{array}{c}C_{2} \\
1\end{array}$ & $\begin{array}{l}C_{4} \\
1-1\end{array}$ & $\begin{array}{l}\left(C_{2}\right)^{2} \\
1-12\end{array}$ & & $\begin{array}{l}C_{8} \\
1-1_{3}-1_{5}\end{array}$ & $-1_{7}$ & $\begin{array}{ll}Q_{8} & \\
1 & -12\end{array}$ & $\begin{array}{l}D_{8} \\
1-1\end{array}$ \\
\hline $\begin{array}{ll}1 & 1\end{array}$ & 1 & & & & & & & & \\
\hline $\begin{array}{ll}C_{2} & 1\end{array}$ & 2 & 1 & & & & & & & \\
\hline $\begin{array}{lr}C_{4} & 1 \\
& -1\end{array}$ & 3 & 2 & $\begin{array}{ll}1 & \\
& \\
& 1\end{array}$ & & & & & & \\
\hline $\begin{array}{lr}\left(C_{2}\right)^{2} & 1 \\
& -1 \\
& 2\end{array}$ & 3 & $\begin{array}{l}2 \\
2\end{array}$ & & \begin{tabular}{|lll}
1 & & \\
& 1 & \\
& & 1
\end{tabular} & & & & & \\
\hline $\begin{array}{lr}C_{8} & 1 \\
& -1_{3} \\
& -1_{5} \\
& -1_{7}\end{array}$ & 4 & 3 & 2 & & & $\begin{array}{lll}1 & & \\
& 1 & \\
& & 1\end{array}$ & 1 & & \\
\hline $\begin{array}{lr}Q_{8} & 1 \\
& -1 \\
& 2\end{array}$ & 4 & $\begin{array}{l}3 \\
2\end{array}$ & $\begin{array}{l}1 \\
1\end{array}$ & $\begin{array}{lll}1 & & \\
& 1 & \\
& & 1\end{array}$ & & & & $\begin{array}{lll}1 & & \\
& 1 & \\
& & \\
& & 1\end{array}$ & \\
\hline $\begin{array}{rr}D_{8} & 1 \\
& -1\end{array}$ & $\begin{array}{l}6 \\
2\end{array}$ & $\begin{array}{l}7 \\
4\end{array}$ & 1 & 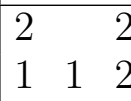 & & & & & $\begin{array}{ll}1 & \\
& 1\end{array}$ \\
\hline
\end{tabular}

Each formal character of $P_{H, V}^{\text {all,1 }}$ is now expressed as a linear combination of the formal characters of the simple functors given in Table 2, and this gives the Cartan matrix. 
TABLE 7. Cartan matrix of $\operatorname{Mack}_{\mathbb{Q}}^{\text {all,1 }}$

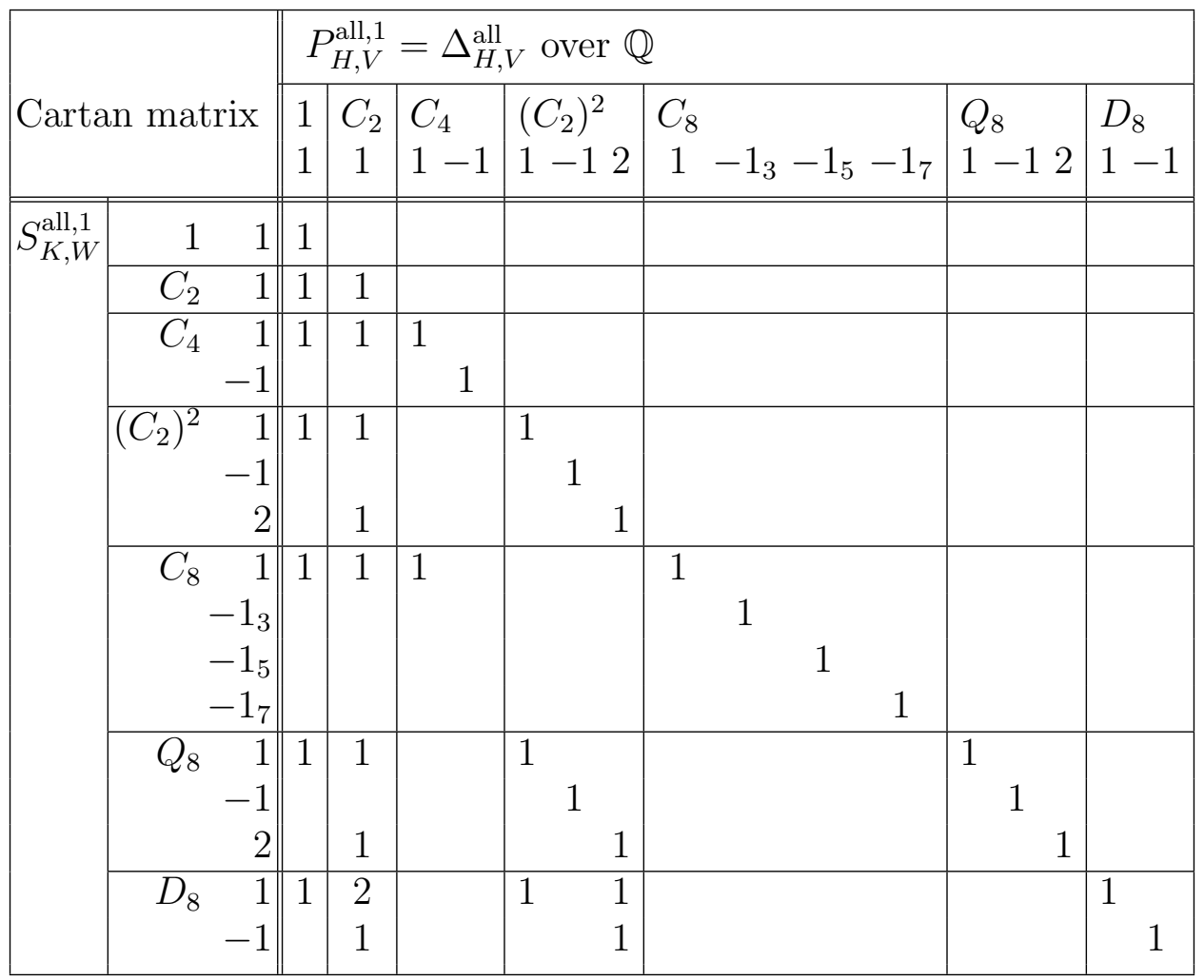

We turn to the situation where $R=\mathbb{F}_{2}$ and compute the matrix $\Psi$ of formal characters of simple functors, then the decomposition matrix from characteristic zero to characteristic 2 , and finally the Cartan matrix in characteristic 2 using Theorem 8.3. The matrix $\Psi$ has in effect been computed previously by people who obtained explicit stable decompositions of classifying spaces of $p$-groups (see [26] for a survey). It was shown in [32, Theorem 6.2] when $H$ is a $p$-group that the multiplicity of a summand parametrized by a pair $(H, V)$ as a stable summand of the $p$-completed classifying space $\left(B G_{+}\right)_{p}^{\wedge}$ equals $\operatorname{dim} S_{H, V}^{\text {all,1 }}(G) / \operatorname{dim} \operatorname{End}_{\mathbb{F}_{p} \text { Out } H}(V)$, and since $R=\mathbb{F}_{p}$ is a splitting field here, this equals $\operatorname{dim} S_{H, V}^{\text {all,1 }}(G)$. The multiplicities of all of these stable summands for the groups we consider here are stated in [6], for example. There is one case where these multiplicities fail to pin down the $\mathbb{F}_{2}$ Out $G$-module structure of $S_{H, V}^{\text {all, }}(G)$ which is the case when $H=C_{2}$ and $G=\left(C_{2}\right)^{2}$, and this is quickly resolved from the description of the simple functor given in [32]. 
TABLE 8. Formal characters of simple functors over $\mathbb{F}_{2}$.

\begin{tabular}{|c|c|c|c|c|c|c|c|}
\hline \multirow[b]{2}{*}{$\Psi$} & \multicolumn{7}{|c|}{$S_{H, V}^{\text {all,1 }}$ over $\mathbb{F}_{2}$} \\
\hline & $\begin{array}{l}1 \\
1\end{array}$ & $\begin{array}{c}C_{2} \\
1\end{array}$ & \begin{tabular}{c|}
$C_{4}$ \\
1
\end{tabular} & $\begin{array}{c}\left(C_{2}\right)^{2} \\
12\end{array}$ & $\begin{array}{c}C_{8} \\
1\end{array}$ & $\begin{array}{c}Q_{8} \\
1\end{array}$ & $\begin{array}{c}D_{8} \\
1\end{array}$ \\
\hline 11 & 1 & & & & & & \\
\hline$C_{2} 1$ & 1 & 1 & & & & & \\
\hline$C_{4} 1$ & 1 & & 1 & & & & \\
\hline $\begin{array}{r}\left(C_{2}\right)^{2} 1 \\
2\end{array}$ & 1 & 1 & & $\begin{array}{ll}1 & \\
& 1\end{array}$ & & & \\
\hline$C_{8} 1$ & 1 & & & & 1 & & \\
\hline $\begin{array}{r}Q_{8} 1 \\
2\end{array}$ & 1 & & & & & $\begin{array}{l}1 \\
1\end{array}$ & \\
\hline$D_{8} 1$ & 1 & 2 & & 2 & & & 1 \\
\hline
\end{tabular}

TABLE 9. Decomposition matrix from $\operatorname{Mack}_{\mathbb{Q}}^{\text {all,1 }}$ to $\operatorname{Mack}_{\mathbb{F}_{2}}^{\text {all,1 }}$

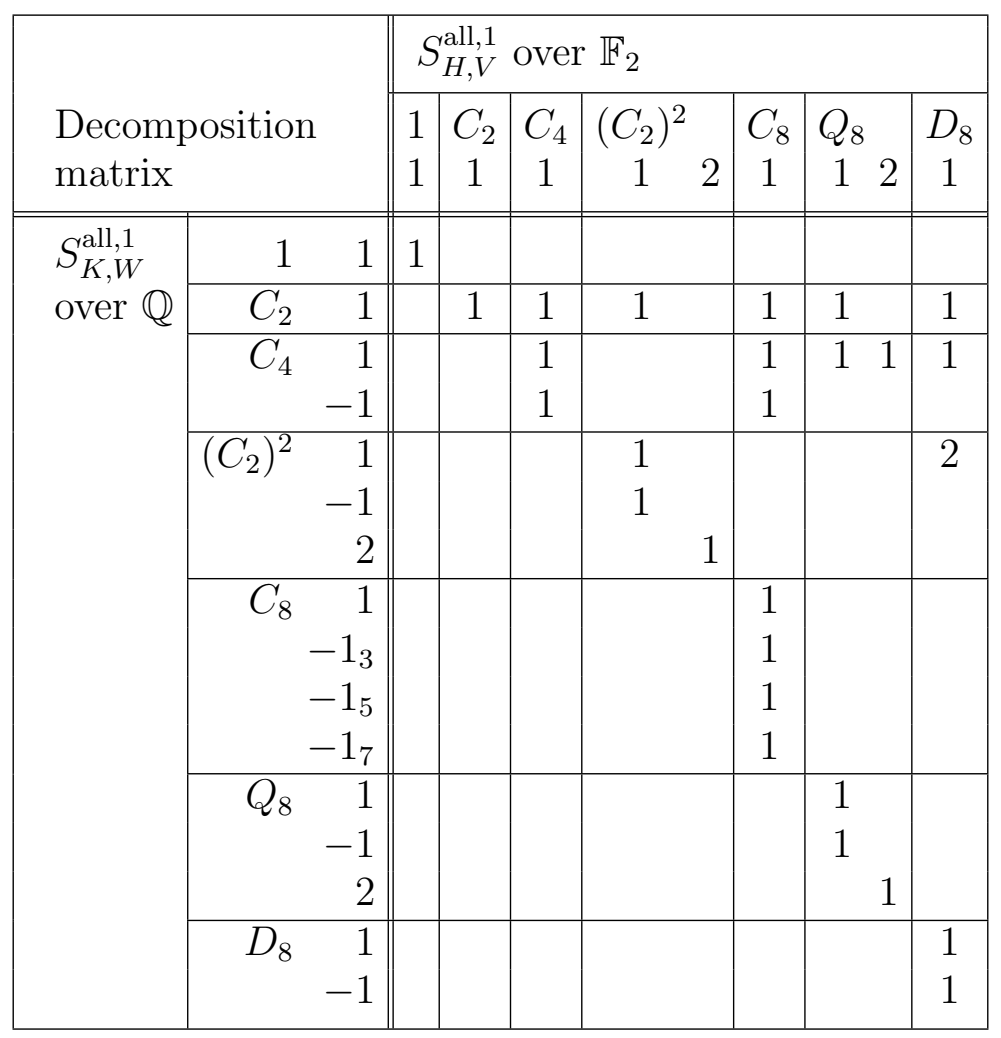


TABLE 10. Cartan matrix of $\mathrm{Mack}_{\mathbb{F}_{2}}^{\text {all,1 }}$

\begin{tabular}{|c|c|c|c|c|c|c|c|c|c|}
\hline \multirow{2}{*}{\multicolumn{2}{|c|}{ Cartan matrix }} & \multicolumn{8}{|c|}{$P_{H, V}^{\text {all, } 1}$ over $\mathbb{F}_{2}$} \\
\hline & & \multirow{2}{*}{\begin{tabular}{|l|}
1 \\
1 \\
1
\end{tabular}} & \multirow{2}{*}{$\begin{array}{c}C_{2} \\
1 \\
0\end{array}$} & \multirow{2}{*}{$\begin{array}{c}C_{4} \\
1 \\
0\end{array}$} & \multirow{2}{*}{\multicolumn{2}{|c|}{ 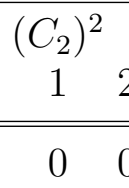 }} & \multirow{2}{*}{$\begin{array}{c}C_{8} \\
1 \\
0\end{array}$} & \multirow{2}{*}{\begin{tabular}{cc}
\multicolumn{2}{l}{$Q_{8}$} \\
1 & 2 \\
0 & 0
\end{tabular}} & \multirow{2}{*}{$\begin{array}{c}D_{8} \\
1 \\
0\end{array}$} \\
\hline \multirow{9}{*}{$\begin{array}{l}S_{K, W}^{\text {all, } 1} \\
\text { over } \mathbb{F}_{2}\end{array}$} & 11 & & & & & & & & \\
\hline & $C_{2} 1$ & 1 & 1 & 1 & 1 & 0 & 1 & 10 & 1 \\
\hline & $C_{4} 1$ & 2 & 2 & 4 & 2 & 0 & 4 & 31 & 3 \\
\hline & $\left(C_{2}\right)^{2} 1$ & 2 & 2 & 2 & 4 & 0 & 2 & 20 & 4 \\
\hline & 2 & 0 & 1 & 1 & 1 & 1 & 1 & 10 & 1 \\
\hline & $C_{8} 1$ & 3 & 3 & 6 & 3 & 0 & 10 & 52 & 5 \\
\hline & $Q_{8} 1$ & 3 & 3 & 4 & 5 & 0 & 4 & & 6 \\
\hline & 2 & 1 & 2 & 3 & 2 & 1 & 3 & 32 & 3 \\
\hline & $D_{8} 1$ & 5 & 7 & 8 & 10 & 2 & 8 & 81 & 16 \\
\hline
\end{tabular}

11.5. Cartan, decomposition and formal character matrices when $\mathcal{X}=\mathcal{Y}=$ all. Fewer of the calculations we need in this situation can be taken from the literature. In [7] the projective functor $P_{1, \mathbb{Q}}$ is identified as the Burnside ring functor and its unique simple quotient $S_{1, \mathbb{Q}}$ as the rational character ring functor. The other composition factors of $P_{1, \mathbb{Q}}$ are also determined in terms of a combinatorial condition, and apart from $S_{1, \mathbb{Q}}$ the only other composition factors indexed

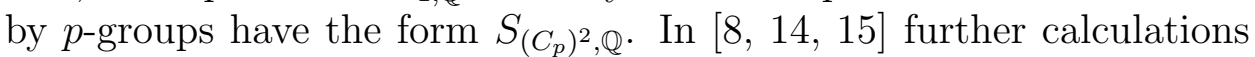
are made of some simple functors over fields of positive characteristic, and these serve as useful checks of our method here, which is the one described in Section 11.2. 
TABLE 11. Formal characters of simple functors $S_{H, V}^{\text {all,all }}$ over $\mathbb{Q}$

\begin{tabular}{|c|c|c|c|c|c|c|c|}
\hline \multirow{3}{*}{$\Psi$} & \multicolumn{7}{|c|}{$S_{H, V}^{\text {all,all }}$ over $\mathbb{Q}$} \\
\hline & 1 & $C_{2}$ & $C_{4}$ & $\left(C_{2}\right)^{2}$ & $C_{8}$ & $Q_{8}$ & $D_{8}$ \\
\hline & & 1 & & & & & \\
\hline $\begin{array}{ll}1 & 1\end{array}$ & 1 & & & & & & \\
\hline $\begin{array}{ll}C_{2} & 1\end{array}$ & \begin{tabular}{|l|}
2 \\
\end{tabular} & 1 & & & & & \\
\hline $\begin{array}{lr}C_{4} & 1 \\
& -1\end{array}$ & 3 & 2 & $\begin{array}{ll}1 & \\
& 1\end{array}$ & & & & \\
\hline $\begin{array}{lr}\left(C_{2}\right)^{2} & 1 \\
& -1 \\
& 2\end{array}$ & \begin{tabular}{|l|}
2 \\
1
\end{tabular} & $\begin{array}{l}2 \\
2\end{array}$ & & $\begin{array}{|lll|}1 & & \\
& 1 & \\
& & \\
& & 1\end{array}$ & & & \\
\hline $\begin{array}{cr}C_{8} & 1 \\
-1_{3} \\
-1_{5} \\
-1_{7}\end{array}$ & 4 & 3 & 2 & & \begin{tabular}{|lll}
1 & & \\
& 1 & \\
& & 1
\end{tabular} & & \\
\hline $\begin{array}{rr}Q_{8} & 1 \\
& -1 \\
& 2\end{array}$ & \begin{tabular}{|l|}
3 \\
1
\end{tabular} & $\begin{array}{l}3 \\
2 \\
2\end{array}$ & $\begin{array}{l}1 \\
1\end{array}$ & $\begin{array}{lll}1 & & \\
& 1 & \\
& & \\
& & 1\end{array}$ & & $\begin{array}{llll}1 & & \\
& & \\
& & \\
& & \\
& & 1\end{array}$ & \\
\hline $\begin{array}{rr}D_{8} & 1 \\
& -1\end{array}$ & \begin{tabular}{|l|}
4 \\
1
\end{tabular} & $\begin{array}{l}7 \\
4\end{array}$ & 1 & $\begin{array}{lll}2 & & 2 \\
1 & 1 & 2\end{array}$ & & & $\begin{array}{ll}1 & \\
& 1\end{array}$ \\
\hline
\end{tabular}


TABLE 12. Composition factor multiplicities in $\operatorname{Mack}_{\mathbb{Q}}^{\text {all,all }}$ of $\Delta_{H, V}^{\text {all }}$

\begin{tabular}{|c|c|c|c|c|c|c|c|c|}
\hline \multirow{2}{*}{\multicolumn{2}{|c|}{$\left[\Delta_{H, V}^{\mathrm{all}}, S_{K, W}^{\text {all,all }}\right]$}} & \multicolumn{7}{|c|}{$\Delta_{H, V}^{\text {all }}$ over $\mathbb{Q}$} \\
\hline & & \multirow{2}{*}{\begin{tabular}{l|}
1 \\
1 \\
1
\end{tabular}} & \multirow{2}{*}{$\begin{array}{c}C_{2} \\
1 \\
\end{array}$} & \multirow{2}{*}{$\begin{array}{l}C_{4} \\
1-1\end{array}$} & \multirow{2}{*}{$\begin{array}{l}\left(C_{2}\right)^{2} \\
1-12\end{array}$} & $\begin{array}{ll}C_{8} & \\
1-1_{3} & -1_{5}-1_{7}\end{array}$ & \multirow{2}{*}{$\begin{array}{l}Q_{8} \\
1-12\end{array}$} & \multirow{2}{*}{$\begin{array}{l}D_{8} \\
1-1\end{array}$} \\
\hline \multirow[t]{7}{*}{$S_{K, W}^{\text {all,all }}$} & $\begin{array}{ll}1 & 1\end{array}$ & & & & & & & \\
\hline & $C_{2} \quad 1$ & & 1 & & & & & \\
\hline & $\begin{array}{lr}C_{4} & 1 \\
& -1\end{array}$ & & & $\begin{array}{ll}1 & \\
& 1\end{array}$ & & & & \\
\hline & $\begin{array}{lr}\left(C_{2}\right)^{2} & 1 \\
& -1 \\
& 2\end{array}$ & 1 & & & $\begin{array}{lll}1 & & \\
& 1 & \\
& & 1\end{array}$ & & & \\
\hline & $\begin{array}{rr}C_{8} & 1 \\
& -1_{3} \\
& -1_{5} \\
& -1_{7}\end{array}$ & & & & & $\begin{array}{ll}1 & \\
& 1\end{array}$ & & \\
\hline & $\begin{array}{lr}Q_{8} & 1 \\
& -1 \\
& 2\end{array}$ & & & & & & \begin{tabular}{|lll}
1 & & \\
& 1 & \\
& & \\
& & 1
\end{tabular} & \\
\hline & $\begin{array}{lr}D_{8} & 1 \\
& -1\end{array}$ & & & & & & & $\begin{array}{|ll|}1 & \\
& 1 \\
& \end{array}$ \\
\hline
\end{tabular}

At this point we record a consequence of the calculation of the composition factors of the $\Delta$ functors.

Proposition 11.2. Let $R$ be a field of characteristic zero, and let $H, K \in\left\{1, C_{2}, C_{4}, C_{8},\left(C_{2}\right)^{2}, Q_{8}, D_{8}\right\}$.

(1) The projective functors $P_{H, V}^{\text {all,all }}$ in $\mathrm{Mack}_{R}^{\text {all,all }}$ are equal to the corresponding functors $\Delta_{H, V}^{\text {all }}$ except in the case $(H, V)=\left(\left(C_{2}\right)^{2}, 1\right)$.

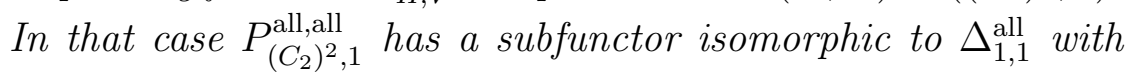
quotient $\Delta_{\left(C_{2}\right)^{2}, 1}^{\text {all }}$. In the cases when $P_{H, V}^{\text {all,all }}=\Delta_{H, V}^{\text {all }}$ it follows that $P_{H, V}^{\text {all,all }}(G)=0$ unless $G$ has $H$ as a section.

(2) We have $\operatorname{dim} \operatorname{Ext}_{\mu_{R}^{\text {all,all }}}^{1}\left(S_{H, V}^{\text {all,all }}, S_{K, W}^{\text {all,all }}\right)=0$ unless one of $(H, V)$ and $(K, W)$ is $(1,1)$ and the other is $\left(\left(C_{2}\right)^{2}, 1\right)$, in which case the dimension is 1 .

Proof. (1) This follows from Lemma 8.1 and BGG reciprocity, which show that the composition factor multiplicity $\left[\Delta_{H, V}^{\text {all }}: S_{K, W}^{\text {all,all }}\right]$ equals the 
filtration multiplicity $\left[P_{K, W}^{\text {all,all }}: \Delta_{H, V}^{\text {all }}\right]$. For a given $K$ this can only be non-zero when $H$ is a section of $K$, and so Table 12 gives complete information about the non-zero multiplicities under consideration.

(2) We apply Theorem 5.10 and duality. 
TABLE 13. Cartan matrix of $\operatorname{Mack}_{\mathbb{Q}}^{\text {all,all }}$

\begin{tabular}{|c|c|c|c|c|c|c|c|c|}
\hline \multirow{2}{*}{\multicolumn{2}{|c|}{ Cartan matrix }} & \multicolumn{7}{|c|}{$P_{H, V}^{\text {all,all }}$ over $\mathbb{Q}$} \\
\hline & & \multirow{2}{*}{\multicolumn{2}{|c|}{\begin{tabular}{c|c}
1 & $C_{2}$ \\
1 & 1 \\
1 &
\end{tabular}}} & \multirow[t]{2}{*}{$\begin{array}{l}C_{4} \\
1-1\end{array}$} & \multirow{2}{*}{$\begin{array}{l}\left(C_{2}\right)^{2} \\
1-12 \\
1\end{array}$} & $\begin{array}{l}C_{8} \\
1-1_{3}-1_{5}-1_{7}\end{array}$ & \multirow[t]{2}{*}{$\begin{array}{l}Q_{8} \\
1-12\end{array}$} & \multirow[t]{2}{*}{$\begin{array}{l}D_{8} \\
1-1\end{array}$} \\
\hline \multirow{7}{*}{$S_{K, W}^{\text {all,all }}$} & $\begin{array}{ll}1 & 1\end{array}$ & & & & & & & \\
\hline & $C_{2} \quad 1$ & & 1 & & & & & \\
\hline & $\begin{array}{lr}C_{4} & 1 \\
& -1\end{array}$ & & & $\begin{array}{ll}1 \\
4\end{array}$ & & & & \\
\hline & $\begin{array}{lr}\left(C_{2}\right)^{2} & 1 \\
& -1 \\
& 2\end{array}$ & 1 & & & $\begin{array}{lll}2 & & \\
& 1 & \\
& & 1\end{array}$ & & & \\
\hline & $\begin{array}{cr}C_{8} & 1 \\
& -1_{3} \\
& -1_{5} \\
& -1_{7}\end{array}$ & & & & & $\begin{array}{lll}1 & & \\
& 1 & \\
& & 1\end{array}$ & & \\
\hline & $\begin{array}{lr}Q_{8} & 1 \\
& -1 \\
& 2\end{array}$ & & & & & & \begin{tabular}{|lll}
1 & & \\
& 1 & \\
& & 1
\end{tabular} & \\
\hline & $\begin{array}{rr}D_{8} & 1 \\
& -1\end{array}$ & & & & & & & $\begin{array}{ll}1 & \\
& 1\end{array}$ \\
\hline
\end{tabular}

TABLE 14. Formal characters of simple functors over $\mathbb{F}_{2}$.

\begin{tabular}{|c|c|c|c|c|c|c|c|}
\hline \multirow[b]{2}{*}{$\Psi$} & \multicolumn{7}{|c|}{$S_{H, V}^{\text {all,all }}$ over $\mathbb{F}_{2}$} \\
\hline & $\begin{array}{l}1 \\
1\end{array}$ & $\begin{array}{c}C_{2} \\
1\end{array}$ & $\begin{array}{c}C_{4} \\
1\end{array}$ & $\begin{array}{c}\left(C_{2}\right)^{2} \\
1\end{array}$ & $\begin{array}{c}C_{8} \\
1\end{array}$ & $\begin{array}{c}Q_{8} \\
12\end{array}$ & $\begin{array}{c}D_{8} \\
1\end{array}$ \\
\hline 11 & 1 & & & & & & \\
\hline$C_{2} 1$ & 2 & 1 & & & & & \\
\hline$C_{4} 1$ & 2 & 1 & 1 & & & & \\
\hline$\left(C_{2}\right)^{2} 1$ & 2 & 2 & & 1 & & & \\
\hline 2 & 1 & 2 & & 1 & & & \\
\hline$C_{8} 1$ & 2 & 1 & 1 & & 1 & & \\
\hline$Q_{8} 1$ & 2 & 2 & & 1 & & 1 & \\
\hline 2 & 1 & 2 & & & & 1 & \\
\hline$D_{8} 1$ & 5 & 9 & & 1 & & & 1 \\
\hline
\end{tabular}


TABLE 15. Decomposition matrix from $\operatorname{Mack}_{\mathbb{Q}}^{\text {all,all }}$ to $\mathrm{Mack}_{\mathbb{F}_{2}}^{\text {all,all }}$

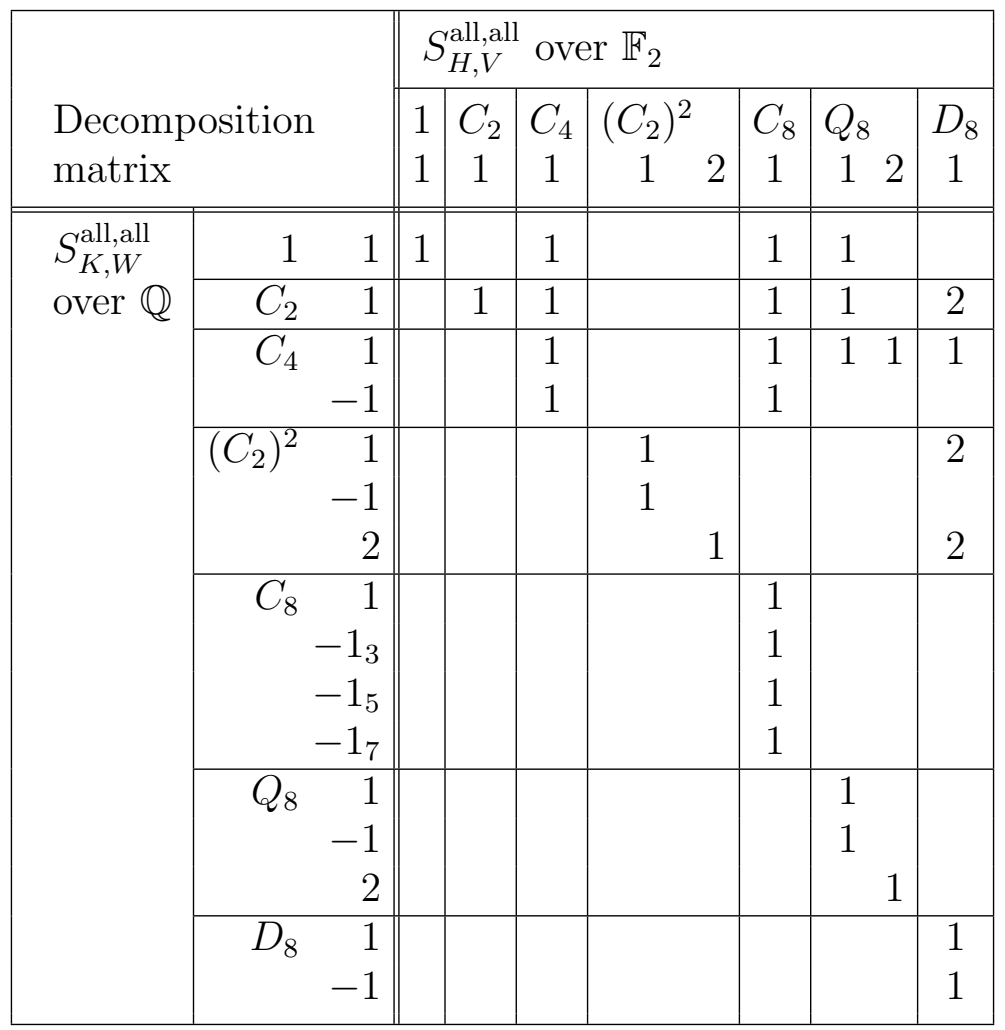

TABLE 16. Cartan matrix of $\mathrm{Mack}_{\mathbb{F}_{2}}^{\text {all,all }}$

\begin{tabular}{|c|c|c|c|c|c|c|c|c|c|}
\hline \multirow{2}{*}{\multicolumn{2}{|c|}{ Cartan matrix }} & \multicolumn{8}{|c|}{$P_{H, V}^{\text {all,all }}$ over $\mathbb{F}_{2}$} \\
\hline & & \multirow{2}{*}{\begin{tabular}{|l|}
1 \\
1 \\
1
\end{tabular}} & \multirow{2}{*}{$\begin{array}{c}C_{2} \\
1 \\
0\end{array}$} & \multirow{2}{*}{\begin{tabular}{|c|}
$C_{4}$ \\
1 \\
1
\end{tabular}} & \multirow{2}{*}{$\begin{array}{c}\left(C_{2}\right)^{2} \\
1 \\
1\end{array}$} & \multirow{2}{*}{\multicolumn{2}{|c|}{ 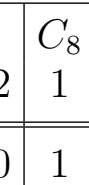 }} & \multirow{2}{*}{\begin{tabular}{ll}
\multicolumn{2}{l}{$Q_{8}$} \\
1 & 2 \\
1 & 0
\end{tabular}} & \multirow{2}{*}{$\begin{array}{c}D_{8} \\
1 \\
2\end{array}$} \\
\hline \multirow{9}{*}{$\begin{array}{l}S_{K, W} \\
\text { over } \mathbb{F}_{2}\end{array}$} & 11 & & & & & 0 & & & \\
\hline & $C_{2} 1$ & 0 & 1 & 1 & 0 & 0 & 1 & 10 & 2 \\
\hline & $C_{4} 1$ & 1 & 1 & 4 & 1 & 0 & 4 & $\begin{array}{ll}3 & 1 \\
\end{array}$ & 5 \\
\hline & $\left(C_{2}\right)^{2} 1$ & 1 & 0 & 1 & 3 & 0 & 1 & $\begin{array}{ll}1 & 0\end{array}$ & 4 \\
\hline & 2 & 0 & 0 & 0 & 0 & 1 & 0 & $\begin{array}{ll}0 & 0\end{array}$ & 2 \\
\hline & $C_{8} 1$ & 1 & 1 & 4 & 1 & 0 & 8 & $\begin{array}{ll}3 & 1 \\
\end{array}$ & 5 \\
\hline & $Q_{8} 1$ & 1 & 1 & 3 & 1 & U & 3 & $\begin{array}{ll}5 & 1\end{array}$ & 5 \\
\hline & 2 & 0 & 0 & 1 & 0 & 0 & 1 & 12 & 1 \\
\hline & $D_{8} 1$ & 2 & 2 & 5 & 4 & 2 & 5 & & 19 \\
\hline
\end{tabular}




\section{REFERENCES}

[1] J.F. Adams, J.H.C. Gunawardena and H. Miller, The Segal conjecture for elementary abelian p-groups, Topology 24 (1985), 435-460.

[2] J.L. Alperin, Local representation theory, Cambridge University Press 1986.

[3] M. Auslander, I. Reiten and S.O. Smalø, Representation theory of Artin algebras, Cambridge studies in advanced mathematics 36, Cambridge University Press 1995.

[4] L. Barker, Rhetorical biset functors, rational p-biset functors and their semisimplicity in characteristic zero, preprint.

[5] D.J. Benson, Representations and cohomology I: basic representation theory of finite groups and associative algebras, Cambridge studies in advanced mathematics 30, Cambridge University Press 1991.

[6] D.J. Benson and M. Feshbach, Stable splittings of classifying spaces of finite groups, Topology 31 (1992), 157-176.

[7] S. Bouc, Foncteurs d'ensembles munis d'une double action, J. Algebra 183 (1996), 664-736.

[8] S. Bouc, The functor of rational representations for p-groups, Adv. Math. 186 (2004), 267-306.

[9] S. Bouc, The Dade group of a p-group, Invent. Math. 164 (2006), 189-231.

[10] S. Bouc, The functor of units of Burnside rings for p-groups, Comment. Math. Helv. 82 (2007), 583-615.

[11] S. Bouc, Rational p-biset functors, J. Algebra 319 (2008), 1776-1800.

[12] S. Bouc and J. Thévenaz, The group of endo-permutation modules, Invent. Math. 139 (2000), 275-349.

[13] N. Bourbaki, Algebra I, Springer-Verlag (1989).

[14] I. Bourizk, Sur des foncteurs simples, Pacific J. Math. 215 (2004), 201-221.

[15] I. Bourizk, A remark on a functor of rational representations, Bull. Belg. Math. Soc. Simon Stevin 13 (2006), 149-157.

[16] E. Cline, B. Parshall and L. Scott, Finite dimensional algebras and highest weight categories, J. reine angew. Math. 391 (1988), 85-99.

[17] E. Cline, B. Parshall and L. Scott, Stratifying endomorphism algebras, Mem. Amer. Math. Soc. 124 (1996), no. 591.

[18] T. tom Dieck, Transformation Groups, Walter de Gruyter, Berlin - New York 1987.

[19] V. Dlab and C.M. Ringel, The module theoretical approach to quasi-hereditary algebras, in 'Representations of Algebras and Related Topics,' (H. Tachikawa and S. Brenner, eds.), pp. 200-224. London Mathematical Society Lecture Note Series, vol 168 (1992).

[20] K. Erdmann, Symmetric groups and quasi-hereditary algebras, pp. 123-161 in V. Dlab and L.L. Scott (eds.), Finite Dimensional Algberas and Related Topics, Kluwer 1994.

[21] P. Gabriel, Des catégories abéliennes, Bull. Soc. Math. Franc. 90 (1962), 323448.

[22] M. Geck and R. Rouquier, Centers and simple modules for Iwahori-Hecke algebras, pp. 251-272 in Finite reductive groups (Luminy, 1994), Progr. Math. 141, Birkhuser Boston, Boston, MA, 1997.

[23] J.A. Green, Polynmial representations of $G L_{n}$, Lecture Notes in Math. 830, Springer-Verlag 1980. 
[24] L.G. Lewis, Jr., The theory of Green functors, unpublished notes, 1981.

[25] L.G. Lewis, Jr., J.P. May and J.E. McClure, Classifying G-spaces and the Segal conjecture, pp. 165-179 in: R.M. Kane et al (eds.), Current Trends in Algebraic Topology, CMS Conference Proc. 2 (1982).

[26] J.R. Martino, Classifying spaces and their maps, pp. 161-198 in 'Homotopy theory and its applications' (Cocoyoc 1993), Contemp. Math. 188, American Math. Soc. 1995.

[27] H. Miller, Letter to J.F. Adams, 1981.

[28] P. Symonds, A splitting principle for group representations, Comment. Math. Helv. 66 (1991), 169-184.

[29] J. Thévenaz, $G$-Algebras and modular representation theory, Oxford University Press 1995.

[30] J. Thévenaz and P.J. Webb, Simple Mackey Functors, Proc. of 2nd international group theory conference, Bressanone (1989), Supplement to Rendiconti del Circolo Matematico di Palermo 23 (1990), 299-319.

[31] J. Thévenaz and P.J. Webb, The structure of Mackey functors, Trans. Amer. Math. Soc. 347 (1995), 1865-1961.

[32] P.J. Webb, Two classifications of simple Mackey functors with applications to group cohomology and the decomposition of classifying spaces, J. Pure Appl. Algebra 88 (1993), 265-304.

[33] P.J. Webb, A guide to Mackey functors, M. Hazewinkel (ed.), Handbook of Algebra vol. 2, Elsevier 2000, pp. 805-836.

[34] P.J. Webb, Stratifications and Mackey functors I: functors for a single group, Proc. London Math. Soc. (3) 82 (2001), 299-336.

[35] P.J. Webb, Weight Theory in the Context of Arbitrary Finite Groups, Fields Institute Communications 40 (2004), 277-289.

[36] P.J. Webb, Standard stratifications of EI categories and Alperin's weight conjecture, to appear in Journal of Algebra.

[37] C.M. Witten, Self-maps of classifying spaces of finite groups and classification of low-dimensional Poincaré duality spaces, Ph.D. thesis, Stanford University 1978.

[38] F. Xu, Homological Properties of Category Algebras, Ph.D. thesis, University of Minnesota 2006.

E-mail address: webb@math.umn.edu

School of Mathematics, University of Minnesota, Minneapolis, MN 55455, USA 